\title{
The existence of conformal metrics with constant scalar curvature and constant boundary mean curvature
}

\author{
ZHENG-CHAO HAN ${ }^{1}$ AND YANYAN LI ${ }^{2}$
}

\section{Introduction.}

Let $(M, g)$ be an $n$ dimensional compact, smooth, Riemannian manifold without boundary. For $n=2$, the Uniformization Theorem of Poincaré says that there exist metrics on $M$ which are pointwise conformal to $g$ and have constant Gauss curvature. For $n \geq 3$, the well known Yamabe conjecture states that there exist metrics on $M$ which are pointwise conformal to $g$ and have constant scalar curvature. The Yamabe conjecture has been proved through the work of Yamabe $[\mathrm{Y}]$, Trudinger $[\mathrm{T}]$, Aubin $[\mathrm{A}]$, and Schoen [S1]. See Lee and Parker [LP] for a survey. See also Bahri and Brezis [BB], Bahri [B], and Schoen [S2-3] for works on the problem and related ones.

Analogues of the Yamabe problem for compact Riemannian manifolds with boundary have been studied by Cherrier, Escobar, and others. In particular, Escobar proved in [E2] that a large class of compact Riemannian manifolds with boundary are conformally equivalent to one with constant scalar curvature and zero mean curvature on the boundary. See also [E3][E5] for related results.

From now on in the paper, $(M, g)$ denotes some smooth compact $n$ dimensional Riemannian manifold with boundary, unless we specify otherwise. We use $M^{\circ}$ to denote the interior of $M$, and $\partial M$ the boundary of $M$. We use $L_{g}$ to denote $\Delta_{g}-c(n) R_{g}$, where $c(n)$ is $\frac{n-2}{4(n-1)}, B_{g}$ to denote $\frac{\partial}{\partial \nu}+\frac{n-2}{2} h_{g}$, where $\nu$ is the outward unit normal on $\partial M$ with respect to $g$, and $h_{g}$ to denote the mean curvature of $\partial M$ with respect to the inner normal (balls in $\mathbf{R}^{n}$ have positive mean curvatures).

\footnotetext{
${ }^{1}$ Partially supported by NSF grant DMS-9704488, a Rutgers University Research Council Grant and a Rutgers University Minority Faculty Development Grant.

${ }^{2}$ Partially supported by the Alfred P. Sloan Foundation Research Fellowship and NSF grant DMS-9706887.
} 
Let $u>0$ be some positive function on $M$, and consider the metric $\tilde{g}=u^{4 /(n-2)} g$. The scalar curvature $R_{\tilde{g}}$ can be calculated as

$$
R_{\tilde{g}}=-\frac{4(n-1)}{n-2} u^{-\frac{n+2}{n-2}} L_{g} u
$$

and the mean curvature $h_{\tilde{g}}$ can be calculated as

$$
h_{\tilde{g}}=\frac{2}{n-2} u^{-\frac{n}{n-2}} B_{g} u .
$$

Thus the boundary value problem

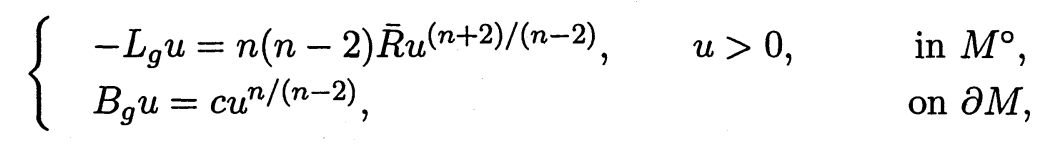

for some constants $\bar{R}$ and $c$, is equivalent to saying that $(M, \tilde{g})$ has constant scalar curvature in $M^{\circ}$ and constant mean curvature on $\partial M$. We remark that $\vec{R}$ can be taken to be 0 , or \pm 1 after scaling.

Consider the following eigenvalue problem on $(M, g)$ :

$$
\begin{cases}-L_{g} \varphi=\lambda \varphi, & \text { in } M^{\circ} \\ B_{g} \varphi=0, & \text { on } \partial M\end{cases}
$$

Let $\lambda_{1}(M)$ denote the first eigenvalue. It is well know that

$$
\lambda_{1}(M)=\min _{\varphi \in H^{1}(M) \backslash\{0\}} \frac{\int_{M}\left(|\nabla \varphi|^{2}+c(n) R_{g} \varphi^{2}\right)+\frac{n-2}{2} \int_{\partial M} h_{g} \varphi^{2}}{\int_{M} \varphi^{2}} .
$$

We say that a manifold $M$ is of positive (negative, zero) type if $\lambda_{1}(M)>0$ $(<0,=0)$. This notion is conformally invariant. The $\bar{R}$ will be scaled to 1 , -1 , or 0 , according to whether $M$ is of positive, negative, or zero type. We will use $\mathcal{M}_{c}$ to denote the set of solutions of (1.1) in $C^{2}(M)$.

Consider

$$
Q(\varphi)=\frac{\int_{M}\left(\left|\nabla_{g} \varphi\right|^{2}+c(n) R_{g} \varphi^{2}\right)}{\left(\int_{M}|\varphi|^{\frac{2 n}{n-2}}\right)^{\frac{n-2}{n}}}
$$

for $\varphi \in H^{1}(M) \backslash\{0\}$. It is clear that, up to some harmless positive constant, $\varphi \in \mathcal{M}_{0}$ for any positive critical point of the functional $Q$.

The Sobolev quotient of $(M, g)$ is given by

$$
Q(M, g)=\inf \left\{Q(\varphi) \mid \varphi \in H^{1}(M) \backslash\{0\}\right\} .
$$


It is clear that $Q(M, g)$ is positive if the first eigenvalue of $-L_{g}$ is positive, is negative if the first eigenvalue of $-L_{g}$ is negative, and is zero if the first eigenvalue of $-L_{g}$ is zero.

Cherrier proved in [C] that, similar to the Yamabe problem, $Q(M, g)$ is achieved if

$$
Q(M, g)<Q\left(\mathbf{S}_{+}^{n}, g_{0}\right)
$$

where $\left(\mathbf{S}_{+}^{n}, g_{0}\right)$ denotes the standard half sphere. In the same paper he also showed the regularity of solutions to such problems. For a large class of manifolds, Escobar established (1.2) in [E2], thus showed $\mathcal{M}_{0} \neq \phi$. In [E3], Escobar obtained existence of solutions of (1.1) for the case of $\bar{R}=0$ and $c$ an arbitrary constant. More recently, Escobar showed in [E4] that, under the same hypotheses as in [E2], there exist $c^{+}>0$ and $c^{-}<0$ such that $\mathcal{M}_{c^{+}} \neq \phi$ and $\mathcal{M}_{c^{-}} \neq \phi$. Naturally one wonders whether $\mathcal{M}_{c} \neq \phi$ for all $c \in \mathbf{R}^{n}$. We proposed in [HL1] two conjectures concerning this. Before stating the conjectures and the main result there, we first give the following natural subcritical approximation of (1.1), introduced in [HL1],

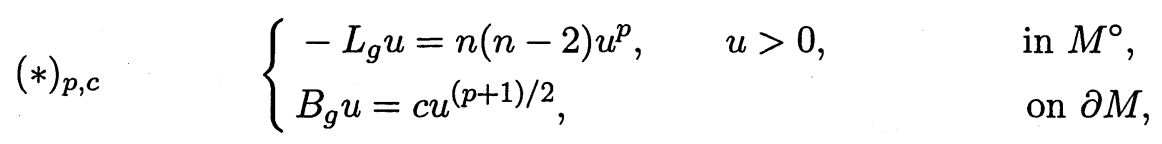

here $c \in \mathbf{R}$ and $1<p \leq(n+2) /(\dot{n}-2)$. Let $\mathcal{M}_{p, c}$ denote the set of solutions of $(*)_{p, c}$ in $C^{2}(M)$. Here, we have set $\bar{R}=1$ to restrict ourselves to the case for manifolds of positive type. As is well known, the existence problems are more difficult for this case.

Conjecture 1. Let $(M, g)$ be a smooth compact n dimensional Riemannian manifold with boundary of positive type. Then for all $c \in \mathbf{R}, \mathcal{M}_{c} \neq \phi$.

Conjecture 2. Let $(M, g)$ be a smooth compact n dimensional Riemannian manifold with boundary of positive type which is not conformally equivalent to the standard half sphere. Then for all $\bar{c}>0$, there exist positive constants $\delta_{0}=\delta_{0}(M, g, \bar{c})$ and $C=C(M, g, \bar{c})>0$ such that

$$
1 / C \leq u(x) \leq C, \quad \forall x \in M ; \quad\|u\|_{C^{2}(M)} \leq C,
$$

for all $u \in\left(\cup_{(n+2) /(n-2)-\delta_{0} \leq p \leq(n+2) /(n-2)} \cup_{|c| \leq \bar{c}} \mathcal{M}_{p, c}\right)$.

We have established in [HL1] both Conjecture 1 and Conjecture 2 when $(M, g)$ is a smooth compact $n(n \geq 3)$ dimensional locally conformally 
flat Riemannian manifold of positive type with umbilic boundary, and have shown in [HL1] that Conjecture 1 can be deduced from Conjecture 2. We recall that $M$ has umbilic boundary if every boundary point is umbilic, i.e., the second fundamental form at the point is a constant multiple of the metric. In this paper, we establish Comjecture 1 when $(M, g)$ is a smooth compact $n(n \geq 5)$ dimensional Riemannian manifold of positve type with at least one non-umbilic point on $\partial M$. More precisely, we have

Theorem 1.1. For $n \geq 5$, let $(M, g)$ be a smooth compact $n$ dimensional Riemannian manifold of positve type with at least one non-umbilic point on $\partial M$. Then $\mathcal{M}_{c} \neq \phi$ for all $c \in \mathbf{R}$.

Remark 1.1. Further existence results will be given in a forthcoming paper [HL3].

In the remaining of this section, we describe our approach to the proof of Theorem 1.1 and the issues involved.

We establish Theorem 1.1 by variational methods. It is easy to verify that a nontrivial critical point of the functional

$$
\begin{aligned}
I(u)= & \frac{1}{2} \int_{M}\left[|\nabla u|^{2}+\frac{n-2}{4(n-1)} R_{g} u^{2}\right]+\frac{n-2}{4} \int_{\partial M} h_{g} u^{2} \\
& -\frac{(n-2)^{2}}{2} \int_{M}\left(u^{+}\right)^{\frac{2 n}{n-2}}-\frac{(n-2)}{2(n-1)} c \int_{\partial M}\left(u^{+}\right)^{\frac{2(n-1)}{n-2}}
\end{aligned}
$$

is a solution of (1.1). It is known that $I \in C^{2}\left(H^{1}(M), \mathbf{R}\right)$.

We find a nontrivial critical point of $I(u)$ using the following Mountain Pass Lemma of Amborsetti and Rabinowitz [AR].

Mountain Pass Lemma (MPL). Let $X$ be a Banach space and $I \in$ $C^{1}(X, \mathbf{R})$. Suppose that $I(0)=0$ and that there exists $0 \neq u_{0} \in X$ such that $I\left(u_{0}\right) \leq 0$. Let $\Gamma$ denote the set of continuous paths in $X$ connecting 0 and $u_{0}$ and define $I_{m p}=\inf _{\gamma \in \Gamma} \sup _{u \in \gamma} I(u)$. Suppose that $I_{m p}>0$ and that $I$ satisfies the (PS) condition at level $I_{m p}$. Then $I_{m p}$ is a critical value of $I$.

The nonlinearities in our functional $I$ are of critical growth. It is known that, in general, the (PS) condition is not satisfied in the presence of such nonlinearities. However, it will be verified in Appendix A (see Lemma 1.2 below) that $I$ satisfies the (PS) condition below certain threshold level $S_{c}$. Recovery of compactness of (PS) sequences below certain threshold level was used by Brezis and Nirenberg in [BN], and has since been used in many 
contexts. Our contribution lies in reducing the verification of $I_{m p}$ 's staying below the threshold to an extremal problem on spherical caps with the standard metric. See the end of this section and the beginning of section 3 for details.

Let us first introduce some quantities and define $S_{c}$. Let

$$
u_{1}(z)=\left(\frac{1}{1+\left|z^{\prime}\right|^{2}+\left|z_{n}-T_{c}\right|^{2}}\right)^{\frac{n-2}{2}},
$$

where $T_{c}=-\frac{c}{n-2}$. Then $u_{1}$ solves

$$
\left\{\begin{aligned}
-\Delta u_{1} & =n(n-2) u_{1}^{(n+2) /(n-2)}, & \text { in } \mathbf{R}_{+}^{n} \\
\frac{\partial u_{1}}{\partial z_{n}} & =-c u_{1}^{n /(n-2)}, & \text { on } z_{n}=0 .
\end{aligned}\right.
$$

We also define

$$
a_{0}=\int_{\mathbf{R}_{+}^{n}}\left|\nabla u_{1}\right|^{2} ; \quad b_{0}=\int_{\mathbf{R}_{+}^{n}} u_{1}^{\frac{2 n}{n-2}} ; \quad \text { and } \quad d_{0}=c \int_{\partial \mathbf{R}_{+}^{n}} u_{1}^{\frac{2(n-1)}{n-2}} .
$$

If we multiply the equation of $u_{1}$ by $u_{1}$ and integrate by parts, we obtain the relation

$$
a_{0}=n(n-2) b_{0}+d_{0} \text {. }
$$

Now we set

$$
S_{c}=\frac{a_{0}}{2(n-1)}+\frac{(n-2)^{2} b_{0}}{2(n-1)} .
$$

This is the threshold level mentioned earlier.

As stated earlier, $I$ satisfies (PS) at levels below $S_{c}$. For simplicity, we do not prove this here, instead we establish the following weaker result which is, as well known, enough in establishing the existence result via (MPL).

Lemma 1.2. Suppose $\lambda_{1}(M)>0$. Let $\left\{u_{i}\right\} \subset H^{1}(M)$ be a sequence of functions satisfying,

$$
I\left(u_{i}\right) \rightarrow b<S_{c}
$$

and

$$
\max _{v \in H^{1}(M) \backslash\{0\}} \frac{\left|I^{\prime}\left(u_{i}\right) v\right|}{\|v\|} \rightarrow 0 .
$$

Then after passing to a subsequence, either $\left\{u_{i}\right\}$ weakly converges in $H^{1}(M)$ to some solution of (1.1) or converges strongly to 0 in $H^{1}(M)$. 
The proof will be deferred to Appendix A. Since we are assuming $\lambda_{1}(M)>0$, it is easy to see that for some $r_{0}>0$ and $\epsilon_{0}>0$, we have $I(u) \geq \epsilon_{0}, \forall u$ with $\|u\|=r_{0}$. For any nonzero $u$ in $H^{1}(M)$, due to the exlicit form of $I, I(t u)<0$ for large $t$. Therefore, for any nonzero $u$, we can take $u_{0}=\bar{t} u$ for sufficiently large $\bar{t}$ and define $I_{m p}$ as in the statement of MPL. All we are left to prove is that

$$
\max _{0<t<\infty} I(t u)<S_{c}
$$

for appropriate choice of $u$.

In the cases to be treated in this paper, i.e., when $\partial M$ is assumed to have a non-umbilic point, we are going to choose a localized test function to achieve (1.7) as follows. In local coordinates near a non-umbilic point of $\partial M$, we choose $u$ in the form of

$$
u(x)=\epsilon^{-\frac{n-2}{2}} \psi(x)\left[u_{1}(x / \epsilon)+\delta \phi(x / \epsilon)\right],
$$

for some appropriate choice of $\phi$, where $\epsilon$ and $\delta$ are small parameters, $\psi$ is a cut-off function to be specified later. For any $u$ given in (1.8), if we take $\phi$ to be smooth with compact support, then we will show that, for $\epsilon, \delta>0$ small, we have

$$
\max _{0<t<\infty} I(t u)=S_{c}+Q_{1} \epsilon \delta+Q_{2} \delta^{2}+Q_{3} \epsilon^{2}+\circ\left(\epsilon^{2}+\delta^{2}\right),
$$

where $Q_{1}$ is a linear functional in $\phi$ given in (2.4), $Q_{2}$ is a quadratic functional in $\phi$ given in (??), and $Q_{3}$ is a number expressed in terms of $n, c$, and geometric data of $\partial M$ at the point, as given in (2.9). We remark that $Q_{2} \geq 0$, for any choice of $\phi$, as will be shown in section 3. It is clear from (1.9) that a sufficient condition to achieve (1.7) is to find a $\phi$ such that

$$
Q_{1}^{2}(\phi)-4 Q_{2}(\phi) Q_{3}>0 .
$$

When $c \leq 0, Q_{3} \leq 0$ from the explicit expressions of $Q_{3}$, and there is an easy choice of $\phi$ to achieve (1.10). For $c>0, Q_{3}>0$, and there is no obvious choice of $\phi$ to achieve (1.10).

The novelty of our systematic search of the test function $u$ in the form (1.8) is to have reduced the search to an extremal problem in the Euclidean half space. It comes from extremizing (1.10) in the form of

$$
4 Q_{3}\left(\inf _{Q_{1}(\phi) \neq 0} \frac{Q_{2}(\phi)}{Q_{1}^{2}(\phi)}\right)<1 .
$$


This leads to the analysis of an eigenvalue problem on spherical caps with Robin type boundary conditions. This is set up as (3.7) in section 3. (1.11) can be expressed in terms of the eigenvalues $\mu_{k}$ 's, as given in (3.8). In section 3 , we obtain recursive formulae for computing the eigenvalues $\mu_{k}$ and the corresponding eigenfunctions. We also prove that the quadratic form $Q_{2}$ is non-negative definite, and identify its kernel. For the verification of (3.8), we need to express the first two terms in (3.8) explicitly in terms of the eigenfunctions associated with $\mu_{2}$ and $\mu_{3}$. We also need some estimate on $\mu_{2}$ and $\mu_{3}$. These are done in section 4 . At the end of section 4 , we complete our proof of Theorem 1.1.

\section{Expressions for $Q_{1}, Q_{2}, Q_{3}$ and the case of $c \leq 0$.}

In this section, we derive the expressions for $Q_{1}, Q_{2}$, and $Q_{3}$. As a preliminary step, we will choose a test function $u$ and compute $\max _{0<t<\infty} I(t u)$. We will specify $u$ later. For the moment, we have, schematically, for $t>0$,

$$
I(t u)=\frac{a}{2} t^{2}-\frac{(n-2)^{2}}{2} b t^{\frac{2 n}{n-2}}-\frac{(n-2)}{2(n-1)} d t^{\frac{2(n-1)}{n-2}},
$$

where

$$
\begin{aligned}
a & =\int_{M}\left[|\nabla u|_{g}^{2}+\frac{n-2}{4(n-1)} R_{g} u^{2}\right] d v_{g}+\frac{n-2}{2} \int_{\partial M} h_{g} u^{2} d v_{\partial M} \\
b & =\int_{M}\left(u^{+}\right)^{\frac{2 n}{n-2}} d v_{M}
\end{aligned}
$$

and

$$
d=c \int_{\partial M}\left(u^{+}\right)^{\frac{2(n-1)}{n-2}} d v_{\partial M} .
$$

Simple calculus shows that

$$
\max _{0<t<\infty} I(t u)=\frac{a t^{2}}{2(n-1)}+\frac{(n-2)^{2} b t^{\frac{2 n}{n-2}}}{2(n-1)},
$$

where $t>0$ solves

$$
a=n(n-2) b t^{\frac{4}{n-2}}+d t^{\frac{2}{n-2}},
$$

from which we obtain

$$
t=\left\{\frac{-d+\sqrt{d^{2}+4 n(n-2) a b}}{2 n(n-2) b}\right\}^{\frac{n-2}{2}} .
$$


We are going to choose $u$ in the form of $\epsilon^{-\frac{n-2}{2}} \psi(x)\left[u_{1}(x / \epsilon)+\delta \phi(x / \epsilon)\right]$ in local coordinates near a non-umbilic point, where $\epsilon$ and $\delta$ are small parameters, $\psi$ is a cut-off function, $\equiv 1$ near the point, and $\phi \in C_{c}^{\infty}\left(\mathbf{R}^{n}\right)$. We will show in the following that, for $n \geq 5$,

$$
\begin{aligned}
& a=a_{0}+A_{0} \delta+A_{1} \epsilon \delta+A_{2} \delta^{2}+A_{3} \epsilon^{2}+o\left(\epsilon^{2}+\delta^{2}\right), \\
& b=b_{0}+B_{0} \delta+B_{1} \epsilon \delta+B_{2} \delta^{2}+B_{3} \epsilon^{2}+o\left(\epsilon^{2}+\delta^{2}\right), \\
& d=d_{0}+D_{0} \delta+D_{1} \epsilon \delta+D_{2} \delta^{2}+D_{3} \epsilon^{2}+o\left(\epsilon^{2}+\delta^{2}\right),
\end{aligned}
$$

where the $A_{i}, B_{i}$, and $D_{i}$ are explicitly given in terms of $\phi$, but independent of $\epsilon, \delta$. From the equation satisfied by $t$, we find the relation

$$
t=1+T_{0} \delta+T_{1} \epsilon \delta+T_{2} \delta^{2}+T_{3} \epsilon^{2}+o\left(\epsilon^{2}+\delta^{2}\right),
$$

where

$$
\begin{aligned}
T_{0} & =\frac{A_{0}-n(n-2) B_{0}-D_{0}}{4 n b_{0}+\frac{2}{n-2} d_{0}} \\
T_{1} & =\frac{A_{1}-n(n-2) B_{1}-D_{1}}{4 n b_{0}+\frac{2}{n-2} d_{0}} \\
T_{2} & = \\
& =\frac{A_{2}-n(n-2) B_{2}-D_{2}-\left(4 n B_{0}+\frac{2}{n-2} D_{0}\right) T_{0}-\left(\frac{2 n(6-n)}{n-2} b_{0}+\frac{4-n}{(n-2)^{2}} d_{0}\right) T_{0}^{2}}{4 n b_{0}+\frac{2}{n-2} d_{0}}
\end{aligned}
$$

and

$$
T_{3}=\frac{A_{3}-n(n-2) B_{3}-D_{3}}{4 n b_{0}+\frac{2}{n-2} d_{0}} .
$$

Putting these into the expansion for $\max _{0<t<\infty} I(t u)$, we obtain

$$
\begin{aligned}
& \text { (2.1) } \max _{0<t<\infty} I(t u) \\
& =S_{c}+\frac{\delta}{2}\left[A_{0}-(n-2)^{2} B_{0}-\frac{n-2}{n-1} D_{0}\right]+\frac{\epsilon \delta}{2}\left[A_{1}-(n-2)^{2} B_{1}-\frac{n-2}{n-1} D_{1}\right] \\
& +\frac{\delta^{2}}{2}\left[A_{2}-(n-2)^{2} B_{2}-\frac{n-2}{n-1} D_{2}+\frac{(n-2)}{2} \frac{\left[A_{0}-n(n-2) B_{0}-D_{0}\right]^{2}}{a_{0}+n(n-2) b_{0}}\right] \\
& +\frac{\epsilon^{2}}{2}\left[A_{3}-(n-2)^{2} B_{3}-\frac{n-2}{n-1} D_{3}\right]+o\left(\epsilon^{2}+\delta^{2}\right) .
\end{aligned}
$$


To evaluate the $A_{i}, B_{i}$, and $D_{i}$ in terms of $\phi$, we first set up convenient coordinate systems. As in [E1], we can assume that $g$ has the property $h(0)=0$, and $R_{i j}(0)=0$. Let $\left(x_{1}, \cdots, x_{n}\right)$ be normal coordinates around $0 \in \partial M$, such that the second fundamental form of $\partial M$ at 0 has a diagonal form. Then $\partial M$ can be expressed near 0 by

$$
x_{n}=f\left(x_{1}, \cdots, x_{n-1}\right)=\sum_{i=1}^{n-1} \frac{1}{2} \lambda_{i} x_{i}^{2}+\sum_{1 \leq i, j, k \leq n-1} a_{i j k} x_{i} x_{j} x_{k}+O\left(\left|x^{\prime}\right|^{4}\right) .
$$

So $\sum_{i=1}^{n-1} \lambda_{i}=0$. Recall that in a normal coordinate, $g^{i j}$ has the following expansion

$$
g^{i j}=\delta^{i j}-\frac{1}{3} R_{i k l j} x_{k} x_{l}+O\left(|x|^{3}\right),
$$

where $R_{i j k l}$ denote the coefficients of the Riemann curvature tensor at 0 ; and $\sqrt{g}=\sqrt{\operatorname{det}\left(g_{i j}\right)}$ has the expansion

$$
\sqrt{g}=1-\frac{1}{6} R_{i j} x_{i} x_{j}+O\left(|x|^{3}\right) .
$$

Let $\rho_{0}$ be a positive number and consider the cylinder

$$
C_{\rho_{0}}=C_{\rho_{0}}(0)=\left\{\left(x_{1}, \cdots, x_{n}\right) \mid x_{1}^{2}+\cdots+x_{n-1}^{2}<\rho_{0}^{2},-\rho_{0}<x_{n}<\rho_{0}\right\}
$$

and

$$
C_{\rho_{0}}^{+}=C_{\rho_{0}}^{+}(0)=\left\{\left(x_{1}, \cdots, x_{n}\right) \in C_{\rho_{0}} \mid x_{n}>0\right\} .
$$

Let $\psi$ be a smooth cut-off function such that $\psi \equiv 1$ on $C_{\rho_{0}}$, is supported in $C_{2 \rho_{0}}$, and $|\nabla \psi| \leq C / \rho_{0},\left|\nabla^{2} \psi\right| \leq C / \rho_{0}^{2}$ for some constant $C$. In the following, we will assume $n \geq 4$ and will choose $u=\psi(x) \epsilon^{-\frac{n-2}{2}}\left[u_{1}(x / \epsilon)+\delta \phi(x / \epsilon)\right]$ and evaluate the $A_{i}, B_{i}, D_{i}, i=0,1,2,3, \epsilon, \delta>0$ will be chosen small, $\psi$ is a cut-off function, and $\phi$ is assumed to have compact support and will be chosen later.

$$
\int_{M}|\nabla u|_{g}^{2} d v_{g}=\int_{C_{\rho_{0}} \cap M}|\nabla u|_{g}^{2} d v_{g}+\int_{M \backslash C_{\rho_{0}}}|\nabla u|_{g}^{2} d v_{g}
$$

We calculate the two integrals above separately. First

$$
\int_{M \backslash C_{\rho_{0}}}|\nabla u|_{g}^{2} d v_{g}=O\left(\rho_{0}^{2-n} \epsilon^{n-2}\right)
$$


by a simple computation. Next

$$
\begin{aligned}
\int_{C_{\rho_{0}} \cap M}|\nabla u|_{g}^{2} d v_{g} & \\
= & \int_{C_{\rho_{0}} \cap M}|\nabla u|^{2} d x_{n} d x^{\prime}-\frac{1}{3} \sum R_{i j k l} \int_{C_{\rho_{0}} \cap M} u_{i} u_{l} x_{j} x_{k} d x_{n} d x^{\prime} \\
& +O\left(\int_{C_{\rho_{0}} \cap M}|x|^{3}|\nabla u|^{2} d x_{n} d x^{\prime}\right) .
\end{aligned}
$$

The first integral gives

$$
\int_{C_{\rho_{0}} \cap M}|\nabla u|^{2} d x_{n} d x^{\prime}=\int_{C_{\rho_{0}}^{+}}|\nabla u|^{2} d x_{n} d x^{\prime}-\int_{B_{\rho_{0}}^{n-1}} \int_{0}^{f\left(x^{\prime}\right)}|\nabla u|^{2} d x_{n} d x^{\prime},
$$

with

$$
\begin{aligned}
& \int_{C_{\rho_{0}}^{+}}|\nabla u|^{2} d x_{n} d x^{\prime} \\
& =\int_{C_{\rho_{0} / \epsilon}^{+}}\left|\nabla u_{1}\right|^{2} d z_{n} d z^{\prime}+\delta^{2} \int_{C_{\rho_{0} / \epsilon}^{+}}|\nabla \phi|^{2} d z_{n} d z^{\prime}+2 \delta \int_{C_{\rho_{0} / \epsilon}^{+}} \nabla u_{1} \cdot \nabla \phi \\
& =a_{0}+\delta^{2} \int_{\mathbf{R}_{+}^{n}}|\nabla \phi|^{2}+2 \delta \int_{\mathbf{R}_{+}^{n}} \nabla u_{1} \cdot \nabla \phi+O\left(\rho_{0}^{2-n} \epsilon^{n-2}\right)+o\left(\delta^{2}+\epsilon^{2}\right),
\end{aligned}
$$

and

$$
\int_{B_{\rho_{0}}^{n-1}} \int_{0}^{f\left(x^{\prime}\right)}|\nabla u|^{2} d x_{n} d x^{\prime}=\int_{B_{\rho_{0} / \epsilon}^{n-1}} \int_{0}^{f\left(\epsilon z^{\prime}\right) / \epsilon}\left\{\left|\nabla u_{1}\right|^{2}+2 \delta \nabla u_{1} \cdot \nabla \phi+\delta^{2}|\nabla \phi|^{2}\right\} .
$$

We evaluate them separately.

$$
\begin{aligned}
& \int_{B_{\rho_{0} / \epsilon}^{n-1}} \int_{0}^{f\left(\epsilon z^{\prime}\right) / \epsilon}\left|\nabla u_{1}\right|^{2} \\
& =\frac{\epsilon}{2} \sum_{i=1}^{n-1} \lambda_{i} \int_{B_{\rho_{0} / \epsilon}^{n-1}} \frac{(n-2)^{2}\left(\left|z^{\prime}\right|^{2}+T_{c}^{2}\right)}{\left(1+\left|z^{\prime}\right|^{2}+T_{c}^{2}\right)^{n}} z_{i}^{2} \\
& \quad+\text { const. } \epsilon^{2} \int_{B_{\rho_{0} / \epsilon}^{n-1}} \frac{(n-2)^{2}\left(\left|z^{\prime}\right|^{2}+T_{c}^{2}\right)}{\left(1+\left|z^{\prime}\right|^{2}+T_{c}^{2}\right)^{n}} z_{i} z_{j} z_{k} \\
& \quad+\frac{(n-2)^{2} \epsilon^{2} T_{c}}{4} \int_{B_{\rho_{0} / \epsilon}^{n-1}} \frac{(n-1)\left(\left|z^{\prime}\right|^{2}+T_{c}^{2}\right)-1}{\left(1+\left|z^{\prime}\right|^{2}+T_{c}^{2}\right)^{n+1}}\left(\sum_{i=1}^{n-1} \lambda_{i} z_{i}^{2}\right)^{2}+E(\epsilon) \\
& =\frac{(n-2)^{2} \epsilon^{2} T_{c}}{4} \int_{\mathbf{R}^{n-1}} \frac{(n-1)\left(\left|z^{\prime}\right|^{2}+T_{c}^{2}\right)-1}{\left(1+\left|z^{\prime}\right|^{2}+T_{c}^{2}\right)^{n+1}}\left(\sum_{i=1}^{n-1} \lambda_{i} z_{i}^{2}\right)^{2}+E(\epsilon),
\end{aligned}
$$


due to $\sum_{i=1}^{n-1} \lambda_{i}=0$ and symmetry, here and in the following $E(\epsilon)$ denotes a quantity with the following estimate

$$
E(\epsilon)=\left\{\begin{array}{lr}
O\left(\epsilon^{3}\right) & \text { when } n \geq 6 \\
O\left(\epsilon^{3} \log \frac{\rho_{0}}{\epsilon}\right) & \text { when } n=5 \\
O\left(\epsilon^{2} \rho_{0}\right) & \text { when } n=4
\end{array}\right.
$$

The other two terms are estimated as

$$
\int_{B_{\rho_{0} / \epsilon}^{n-1}} \int_{0}^{f\left(\epsilon z^{\prime}\right) / \epsilon}|\nabla \phi|^{2}=O(\epsilon)
$$

and

$$
\int_{B_{\rho_{0} / \epsilon}^{n-1}} \int_{0}^{f\left(\epsilon z^{\prime}\right) / \epsilon} \nabla u_{1} \cdot \nabla \phi=\frac{\epsilon}{2} \sum_{i=1}^{n-1} \lambda_{i} \int_{\mathbf{R}^{n-1}} \nabla u_{1}\left(z^{\prime}, 0\right) \cdot \nabla \phi\left(z^{\prime}, 0\right) z_{i}^{2}+o(\epsilon) .
$$

A simple estimate gives

$$
\int_{C_{\rho_{0}} \cap M}|\nabla u|^{2}|x|^{3} d x_{n} d x^{\prime}=E(\epsilon)
$$

and

$$
\begin{aligned}
& (n-2)^{-2} \sum R_{i j k l} \int_{C_{\rho_{0}} \cap M} u_{i} u_{l} x_{j} x_{k} d x_{n} d x^{\prime} \\
& =\epsilon^{2} \int_{C_{\rho_{0} / \epsilon}^{+}}\left\{\sum_{i, l<n} R_{i j k l} \frac{z_{i} z_{j} z_{k} z_{l}}{\left(1+\left|z^{\prime}\right|^{2}+\left|z_{n}-T_{c}\right|^{2}\right)^{n}}\right. \\
& \quad+\sum_{i<n} R_{i j k n} \frac{z_{i} z_{j} z_{k}\left(z_{n}-T_{c}\right)}{\left(1+\left|z^{\prime}\right|^{2}+\left|z_{n}-T_{c}\right|^{2}\right)^{n}}+\sum_{l<n} R_{n j k l} \frac{\left(z_{n}-T_{c}\right) z_{j} z_{k} z_{l}}{\left(1+\left|z^{\prime}\right|^{2}+\left|z_{n}-T_{c}\right|^{2}\right)^{n}} \\
& \left.\quad+\sum_{j, k} R_{n j k n} \frac{\left(z_{n}-T_{c}\right)^{2} z_{j} z_{k}}{\left(1+\left|z^{\prime}\right|^{2}+\left|z_{n}-T_{c}\right|^{2}\right)^{n}}\right\}+E(\epsilon)+o\left(\epsilon^{2}+\delta^{2}\right) .
\end{aligned}
$$

Using the symmetry of the Riemann curvature tensor and $R_{n n}(0)=0$, the first 4 terms of the right hand side above can be combined to become

$$
\begin{aligned}
\epsilon^{2} \int_{C_{\rho_{0} / \epsilon}^{+}}\left\{\sum_{i, j, k, l} R_{i j k l} \frac{z_{i} z_{j} z_{k} z_{l}}{\left(1+\left|z^{\prime}\right|^{2}+\left|z_{n}-T_{c}\right|^{2}\right)^{n}}\right. \\
\left.+\sum_{j, k} R_{n j k n} \frac{\left(T_{c}^{2}-2 T_{c} z_{n}\right) z_{j} z_{k}}{\left(1+\left|z^{\prime}\right|^{2}+\left|z_{n}-T_{c}\right|^{2}\right)^{n}}-2 \sum_{i, j, k} R_{i j k n} \frac{T_{c} z_{i} z_{j} z_{k}}{\left(1+\left|z^{\prime}\right|^{2}+\left|z_{n}-T_{c}\right|^{2}\right)^{n}}\right\} .
\end{aligned}
$$


They all vanish because of the symmetry of the Riemann curvature tensor and $R_{n n}(0)=0$. Therefore

$$
\sum R_{i j k l} \int_{C_{\rho_{0}} \cap M} u_{i} u_{l} x_{j} x_{k} d x_{n} d x^{\prime}=E(\epsilon)+o\left(\epsilon^{2}+\delta^{2}\right)
$$

The other two terms in the expression for $a$ can be estimated simply as

$$
\begin{aligned}
\int_{M} R_{g} u^{2} & =O\left(\int_{M \cap C_{\rho_{0}}}|x| u^{2}\right)+O\left(\int_{\left(C_{2 \rho_{0}} \backslash C_{\rho_{0}}\right) \cap M} \rho_{0} u^{2}\right) \\
& =O\left(\epsilon^{3} \int_{0}^{\rho_{0} / \epsilon}(1+r)^{4-n} d r\right)+O\left(\epsilon^{2} \rho_{0} \int_{\rho_{o} / \epsilon}^{2 \rho_{0} / \epsilon}(1+r)^{3-n} d r\right) \\
& =E(\epsilon),
\end{aligned}
$$

using $R_{g}(0)=0$. Using the fact that $g$ is geodesic normal coordinate near 0 and that $h_{g}(0)=0$, we have

$$
\begin{aligned}
\int_{\partial M} h_{g} u^{2}= & \int_{B_{2 \rho_{0}}^{n-1}} h_{g}\left(x^{\prime}, f\left(x^{\prime}\right)\right) u\left(x^{\prime}, f\left(x^{\prime}\right)\right)^{2} d x^{\prime} \\
& +O\left(\int_{B_{2 \rho_{0}}^{n-1}} h_{g}\left(x^{\prime}, f\left(x^{\prime}\right)\right) u\left(x^{\prime}, f\left(x^{\prime}\right)\right)^{2}\left|x^{\prime}\right|^{2} d x^{\prime}\right) \\
& +E(\epsilon)+o\left(\epsilon^{2}+\delta^{2}\right) \\
= & \int_{B_{2 \rho_{0}}^{n-1}} \sum_{i=1}^{n-1} a_{i} x_{i} u\left(x^{\prime}, f\left(x^{\prime}\right)\right)^{2} \\
& +O\left(\int_{B_{2 \rho_{0}}^{n-1}} u\left(x^{\prime}, f\left(x^{\prime}\right)\right)^{2}\left|x^{\prime}\right|^{2} d x^{\prime}\right)+E(\epsilon)+o\left(\epsilon^{2}+\delta^{2}\right)
\end{aligned}
$$

where in the last estimate we used the Taylor expansion for $h_{g}\left(x^{\prime}, f\left(x^{\prime}\right)\right)$ near $x^{\prime}=0$. Now

$$
\begin{aligned}
O\left(\int_{B_{2 \rho_{0}}^{n-1}} u\left(x^{\prime}, f\left(x^{\prime}\right)\right)^{2}\left|x^{\prime}\right|^{2} d x^{\prime}\right) & =O\left(\int_{B_{2 \rho_{0}}^{n-1}}\left(\frac{\epsilon}{\epsilon^{2}+\left|x^{\prime}\right|^{2}}\right)^{n-2}\left|x^{\prime}\right|^{2} d x^{\prime}\right) \\
& =E(\epsilon)
\end{aligned}
$$


and

$$
\begin{aligned}
& \int_{B_{2 \rho_{0}}^{n-1}} \sum_{i=1}^{n-1} a_{i} x_{i} u\left(x^{\prime}, f\left(x^{\prime}\right)\right)^{2} \\
& =\epsilon^{2} \int_{B_{2 \rho_{0} / \epsilon}^{n-1}} \sum_{i=1}^{n-1} a_{i} z_{i} \psi\left(\epsilon z^{\prime}, f\left(\epsilon z^{\prime}\right)\right)^{2}\left\{u_{1}\left(z^{\prime}, f\left(\epsilon z^{\prime}\right) / \epsilon\right)^{2}\right. \\
& \left.+2 \delta u_{1}\left(z^{\prime}, f\left(\epsilon z^{\prime}\right) / \epsilon\right) \phi\left(z^{\prime}, f\left(\epsilon z^{\prime}\right) / \epsilon\right)+\delta^{2} \phi\left(z^{\prime}, f\left(\epsilon z^{\prime}\right) / \epsilon\right)^{2}\right\} \\
& =\epsilon^{2} \int_{B_{2 \rho_{0} / \epsilon}^{n-1}} \sum_{i=1}^{n-1} a_{i} z_{i} \psi\left(\epsilon z^{\prime}, f\left(\epsilon z^{\prime}\right)\right)^{2} u_{1}\left(z^{\prime}, f\left(\epsilon z^{\prime}\right) / \epsilon\right)^{2} \\
& +O\left(\epsilon^{2} \delta \int_{B_{2 \rho_{0} / \epsilon}^{n-1}} \frac{\left|z^{\prime}\right|}{\left(1+\left|z^{\prime}\right|^{2}\right)^{n-2}} d z^{\prime}\right)+O\left(\epsilon^{2} \delta^{2} \int_{B_{2 \rho_{0} / \epsilon}^{n-1}} \frac{\left|z^{\prime}\right|}{\left(1+\left|z^{\prime}\right|^{2}\right)^{n-2}} d z^{\prime}\right) \\
& =\epsilon^{2} \int_{B_{2 \rho_{0} / \epsilon}^{n-1}} \sum_{i=1}^{n-1} a_{i} z_{i} \psi\left(\epsilon z^{\prime}, f\left(\epsilon z^{\prime}\right)\right)^{2} u_{1}\left(z^{\prime}, f\left(\epsilon z^{\prime}\right) / \epsilon\right)^{2}+o\left(\epsilon^{2}+\delta^{2}\right) .
\end{aligned}
$$

We require that $\psi(x)=\psi(|x|)$ for the following estimate. Using the theorem of the mean on $\psi\left(\epsilon z^{\prime}, f\left(\epsilon z^{\prime}\right)\right)^{2} u_{1}\left(z^{\prime}, f\left(\epsilon z^{\prime}\right) / \epsilon\right)^{2}$ with respect to the last component, we compute the remaining integral above by

$$
\begin{aligned}
\epsilon^{2} \int_{B_{2 \rho_{0} / \epsilon}^{n-1}} \sum_{i=1}^{n-1} a_{i} z_{i} \psi\left(\epsilon z^{\prime}, f\left(\epsilon z^{\prime}\right)\right)^{2} u_{1}\left(z^{\prime}, f\left(\epsilon z^{\prime}\right) / \epsilon\right)^{2} \\
=\epsilon^{2} \int_{B_{2 \rho_{0} / \epsilon}^{n-1}} \sum_{i=1}^{n-1} a_{i} z_{i} \psi\left(\epsilon z^{\prime}, 0\right)^{2} u_{1}\left(z^{\prime}, 0\right)^{2} d z^{\prime} \\
+O\left(\epsilon^{2} \int_{B_{2 \rho_{0} / \epsilon}^{n-1}}\left|z^{\prime}\right| \frac{\left|f\left(\epsilon z^{\prime}\right) / \epsilon\right|^{2}+\left|T_{c}\right|\left|f\left(\epsilon z^{\prime}\right) / \epsilon\right|}{\left(1+\left|z^{\prime}\right|^{2}\right)^{n-1}} d z^{\prime}\right) \\
\quad+O\left(\epsilon^{4} / \rho_{0} \frac{\left|z^{\prime}\right|^{2}\left|f\left(\epsilon z^{\prime}\right) / \epsilon\right|}{\left.\left(1+\left|z^{\prime}\right|^{2}\right)^{n-2}\right)}\right. \\
=0+O\left(\epsilon^{4} \int_{B_{2 \rho_{0} / \epsilon}^{n-1}} \frac{\left|z^{\prime}\right|^{5}}{\left(1+\left|z^{\prime}\right|^{2}\right)^{n-1}} d z^{\prime}\right)+O\left(\epsilon^{3}\left|T_{c}\right| \int_{B_{2 \rho_{0} / \epsilon}^{n-1}} \frac{\left|z^{\prime}\right|^{3}}{\left(1+\left|z^{\prime}\right|^{2}\right)^{n-1}} d z^{\prime}\right) \\
\quad+O\left(\epsilon^{5} / \rho_{0} \int_{B_{2 \rho_{0} / \epsilon}^{n-1}} \frac{\left|z^{\prime}\right|^{4}}{\left(1+\left|z^{\prime}\right|^{2}\right)^{n-2}} d z^{\prime}\right) \\
=E(\epsilon)+o\left(\epsilon^{2}\right),
\end{aligned}
$$


where on the third line of the above computation, we used the symmetry of $\psi u_{1}$ and the oddness of $z_{i}$. Putting these together, we obtain, for $n \geq 5$,

$$
\begin{aligned}
& A_{0}=2 \int_{\mathbf{R}_{+}^{n}} \nabla u_{1} \cdot \nabla \phi, \\
& A_{1}=-\sum_{i=1}^{n-1} \lambda_{i} \int_{\mathbf{R}^{n-1}} \nabla u_{1}\left(z^{\prime}, 0\right) \cdot \nabla \phi\left(z^{\prime}, 0\right) z_{i}^{2}, \\
& A_{2}=\int_{\mathbf{R}_{+}^{n}}|\nabla \phi|^{2}, \\
& A_{3}=-\frac{(n-2)^{2} T_{c}}{4} \int_{\mathbf{R}^{n-1}} \frac{(n-1)\left(\left|z^{\prime}\right|^{2}+T_{c}^{2}\right)-1}{\left(1+\left|z^{\prime}\right|^{2}+T_{c}^{2}\right)^{n+1}}\left(\sum_{i=1}^{n-1} \lambda_{i} z_{i}^{2}\right)^{2} .
\end{aligned}
$$

Next we compute the expansion for $b$. Since $\phi$ is assumed to have compact support, we have $u^{+}=u$ as long as we choose $\delta$ small. Then

$$
\begin{aligned}
b & =\int_{C_{\rho_{0}} \cap M} u^{\frac{2 n}{n-2}} d v_{g}+\int_{M \backslash C_{\rho_{0}}} u^{\frac{2 n}{n-2}} d v_{g} \\
& =\int_{C_{\rho_{0}} \cap M} u^{\frac{2 n}{n-2}} d v_{g}+O\left(\epsilon^{3}\right) .
\end{aligned}
$$

In view of $R_{i j}(0)=0$ and (2.2), it follows that

$$
\begin{aligned}
\int_{C_{\rho_{0}} \cap M} u^{\frac{2 n}{n-2}} d v_{g}= & \int_{C_{\rho_{0}}^{+}} u^{\frac{2 n}{n-2}} d x_{n} d x^{\prime}-\int_{B_{\rho_{0}}^{n-1}} \int_{0}^{f\left(x^{\prime}\right)} u^{\frac{2 n}{n-2}} d x_{n} d x^{\prime} \\
& +O\left(\int_{C_{\rho_{0}} \cap M}|x|^{3} u^{\frac{2 n}{n-2}} d v_{g}\right)+O\left(\epsilon^{3}\right) \\
= & \int_{C_{\rho_{0}}^{+}} u^{\frac{2 n}{n-2}} d x_{n} d x^{\prime}-\int_{B_{\rho_{0}}^{n-1}} \int_{0}^{f\left(x^{\prime}\right)} u^{\frac{2 n}{n-2}} d x_{n} d x^{\prime}+O\left(\epsilon^{3}\right) .
\end{aligned}
$$


The first integral can be computed as

$$
\begin{aligned}
\int_{C_{\rho_{0}}^{+}} & u^{\frac{2 n}{n-2}} \\
& =\int_{C_{\rho_{0} / \epsilon}^{+}}\left(u_{1}+\delta \phi\right)^{\frac{2 n}{n-2}} \\
& =\int_{C_{\rho_{0} / \epsilon}^{+}}\left\{u_{1}^{\frac{2 n}{n-2}}+\delta \frac{2 n}{n-2} u_{1}^{\frac{n+2}{n-2}} \phi+\delta^{2} \frac{n(n+2)}{(n-2)^{2}} u_{1}^{\frac{4}{n-2}} \phi^{2}\right\}+o\left(\epsilon^{2}+\delta^{2}\right) \\
& =\int_{\mathbf{R}_{+}^{n}}\left\{u_{1}^{\frac{2 n}{n-2}}+\delta \frac{2 n}{n-2} u_{1}^{\frac{n+2}{n-2}} \phi+\delta^{2} \frac{n(n+2)}{(n-2)^{2}} u_{1}^{\frac{4}{n-2}} \phi^{2}\right\}+o\left(\epsilon^{2}+\delta^{2}\right),
\end{aligned}
$$

and the second integral can be computed as

$$
\begin{aligned}
\int_{B_{\rho_{0}}^{n-1}} \int_{0}^{f} u^{\frac{2 n}{n-2}}= & \int_{B_{\rho_{0} / \epsilon}^{n-1}} \int_{0}^{f\left(\epsilon z^{\prime}\right) / \epsilon}\left(u_{1}+\delta \phi\right)^{\frac{2 n}{n-2}} \\
= & \frac{n \epsilon \delta}{n-2} \sum_{i=1}^{n-1} \lambda_{i} \int_{\mathbf{R}^{n-1}} u_{1}^{\frac{n+2}{n-2}}\left(z^{\prime}, 0\right) \phi\left(z^{\prime}, 0\right) z_{i}^{2} \\
& +\frac{\epsilon^{2} n T_{c}}{4} \int_{\mathbf{R}^{n-1}} \frac{\left(\sum_{i=1}^{n-1} \lambda_{i} z_{i}^{2}\right)^{2}}{\left(1+\left|z^{\prime}\right|^{2}+T_{c}^{2}\right)^{n+1}} d z^{\prime}+E(\epsilon)+o\left(\epsilon^{2}+\delta^{2}\right) .
\end{aligned}
$$

Consequently, for $n \geq 5$,

$$
\begin{aligned}
& B_{0}=\frac{2 n}{n-2} \int_{\mathbf{R}_{+}^{n}} u_{1}^{\frac{n+2}{n-2}} \phi, \\
& B_{1}=-\frac{n}{n-2} \sum_{i=1}^{n-1} \lambda_{i} \int_{\mathbf{R}^{n-1}} u_{1}^{\frac{n+2}{n-2}}\left(z^{\prime}, 0\right) \phi\left(z^{\prime}, 0\right) z_{i}^{2}, \\
& B_{2}=\frac{n(n+2)}{(n-2)^{2}} \int_{\mathbf{R}_{+}^{n}} u_{1}^{\frac{4}{n-2}} \phi^{2}, \\
& B_{3}=-\frac{n T_{c}}{4} \int_{\mathbf{R}^{n-1}} \frac{\left(\sum_{i=1}^{n-1} \lambda_{i} z_{i}^{2}\right)^{2}}{\left(1+\left|z^{\prime}\right|^{2}+T_{c}^{2}\right)^{n+1}} d z^{\prime} .
\end{aligned}
$$

To compute $d$, we note, using the expansion of $g$ and of $\partial M$ around 0 , that the volume form of $\partial M$ has the following expansion on $\partial M \cap C_{2 \rho_{0}}$,

$$
d \operatorname{vol}_{\partial M}=\left(1+\frac{1}{6} \sum_{i, j=1}^{n-1}\left(R_{n i n j}-R_{i j}\right) x_{i} x_{j}+\frac{1}{2} \sum_{i=1}^{n-1} \lambda_{i}^{2} x_{i}^{2}+o\left(\left|x^{\prime}\right|^{2}\right)\right) d x^{\prime}
$$


Recall again that $R_{i j}(0)=0$, so we have

$$
\begin{aligned}
& d=c \int_{\partial M} u^{\frac{2(n-1)}{n-2}} d \operatorname{vol}_{\partial M} \\
& =c \int_{\partial M \cap C_{\rho_{0}}} u^{\frac{2(n-1)}{n-2}} d \operatorname{vol}_{\partial M}+c \int_{\partial M \backslash C_{\rho_{0}}} u^{\frac{2(n-1)}{n-2}} d \operatorname{vol}_{\partial M} \\
& =c \int_{B_{\rho_{0} / \epsilon}^{n-1}}\left\{\left(u_{1}+\delta \phi\right)\left(z^{\prime}, \frac{f\left(\epsilon z^{\prime}\right)}{\epsilon}\right)\right\}^{\frac{2(n-1)}{n-2}} \\
& \left(1+\frac{\epsilon^{2}}{6} \sum_{i, j=1}^{n-1} R_{n i n j} z_{i} z_{j}+\frac{\epsilon^{2}}{2} \sum_{i=1}^{n-1} \lambda_{i}^{2} z_{i}^{2}\right) d z^{\prime}+\circ\left(\epsilon^{2}+\delta^{2}\right) \\
& =c \int_{B_{\rho_{0} / \epsilon}^{n-1}}\left\{u_{1}^{\frac{2(n-1)}{n-2}}+\frac{2(n-1)}{n-2} u_{1}^{\frac{n}{n-2}} \delta \phi+\frac{(n-1) n}{(n-2)^{2}} u_{1}^{\frac{2}{n-2}} \delta^{2} \phi^{2}\right\} \\
& \left(z^{\prime}, \frac{f\left(\epsilon z^{\prime}\right)}{\epsilon}\right)\left(1+\frac{\epsilon^{2}}{6} \sum_{i, j=1}^{n-1} R_{n i n j} z_{i} z_{j}+\frac{\epsilon^{2}}{2} \sum_{i=1}^{n-1} \lambda_{i}^{2} z_{i}^{2}\right) d z^{\prime}+\circ\left(\epsilon^{2}+\delta^{2}\right) \\
& =c \int_{B_{\rho_{0} / \epsilon}^{n-1}} u_{1}^{\frac{2(n-1)}{n-2}}\left(z^{\prime}, \frac{f\left(\epsilon z^{\prime}\right)}{\epsilon}\right)\left(1+\frac{\epsilon^{2}}{6} \sum_{i, j=1}^{n-1} R_{n i n j} z_{i} z_{j}+\frac{\epsilon^{2}}{2} \sum_{i=1}^{n-1} \lambda_{i}^{2} z_{i}^{2}\right) d z^{\prime} \\
& +\frac{2(n-1) c}{(n-2)} \delta \int_{B_{\rho_{0} / \epsilon}^{n-1}} u_{1}^{\frac{n}{n-2}}\left(z^{\prime}, \frac{f\left(\epsilon z^{\prime}\right)}{\epsilon}\right) \phi\left(z^{\prime}, \frac{f\left(\epsilon z^{\prime}\right)}{\epsilon}\right) d z^{\prime} \\
& +\frac{(n-1) n c}{(n-2)^{2}} \delta^{2} \int_{B_{\rho_{0} / \epsilon}^{n-1}} u_{1}^{\frac{2}{n-2}}\left(z^{\prime}, \frac{f\left(\epsilon z^{\prime}\right)}{\epsilon}\right) \phi^{2}\left(z^{\prime}, \frac{f\left(\epsilon z^{\prime}\right)}{\epsilon}\right) d z^{\prime}+\circ\left(\epsilon^{2}+\delta^{2}\right) \text {. }
\end{aligned}
$$

We calculate the right hand side of the above term by term,

$$
\begin{aligned}
& c \int_{B_{\rho_{0} / \epsilon}^{n-1}} u_{1}^{\frac{2(n-1)}{n-2}}\left(z^{\prime}, \frac{f\left(\epsilon z^{\prime}\right)}{\epsilon}\right) d z^{\prime} \\
& =c \int_{B_{\rho_{0} / \epsilon}^{n-1}}\left\{u_{1}^{\frac{2(n-1)}{n-2}}\left(z^{\prime}, 0\right)+\left.\frac{\partial}{\partial z_{n}}\left[u_{1}\left(z^{\prime}, z_{n}\right)^{\frac{2(n-1)}{n-2}}\right]\right|_{z_{n}=0} \frac{f\left(\epsilon z^{\prime}\right)}{\epsilon}\right. \\
& \left.+\left.\frac{1}{2} \frac{\partial^{2}}{\partial z_{n}^{2}}\left[u_{1}\left(z^{\prime}, z_{n}\right)^{\frac{2(n-1)}{n-2}}\right]\right|_{z_{n}=0}\left[\frac{f\left(\epsilon z^{\prime}\right)}{\epsilon}\right]^{2}\right\} d z^{\prime}+\circ\left(\epsilon^{2}+\delta^{2}\right) \\
& =c \int_{B_{\rho_{0} / \epsilon}^{n-1}}\left\{u_{1}^{\frac{2(n-1)}{n-2}}\left(z^{\prime}, 0\right)+\left.\frac{\partial}{\partial z_{n}}\left[u_{1}\left(z^{\prime}, z_{n}\right)^{\frac{2(n-1)}{n-2}}\right]\right|_{z_{n}=0}\left(\frac{\epsilon}{2} \sum_{i=1}^{n-1} \lambda_{i} z_{i}^{2}\right)\right.
\end{aligned}
$$




$$
\begin{aligned}
& \left.+\left.\frac{1}{2} \frac{\partial^{2}}{\partial z_{n}^{2}}\left[u_{1}\left(z^{\prime}, z_{n}\right)^{\frac{2(n-1)}{n-2}}\right]\right|_{z_{n}=0}\left(\frac{\epsilon}{2} \sum_{i=1}^{n-1} \lambda_{i} z_{i}^{2}\right)^{2}\right\} d z^{\prime}+\circ\left(\epsilon^{2}+\delta^{2}\right) \\
= & d_{0}+\left.\frac{c \epsilon^{2}}{8} \int_{B_{\rho_{0} / \epsilon}^{n-1}} \frac{\partial^{2}}{\partial z_{n}^{2}}\left[u_{1}\left(z^{\prime}, z_{n}\right)^{\frac{2(n-1)}{n-2}}\right]\right|_{z_{n}=0}\left(\sum_{i=1}^{n-1} \lambda_{i} z_{i}^{2}\right)^{2} d z^{\prime}+\circ\left(\epsilon^{2}+\delta^{2}\right) \\
= & d_{0}+\left.\frac{c \epsilon^{2}}{8} \int_{\mathbf{R}^{n-1}} \frac{\partial^{2}}{\partial z_{n}^{2}}\left[u_{1}\left(z^{\prime}, z_{n}\right)^{\frac{2(n-1)}{n-2}}\right]\right|_{z_{n}=0}\left(\sum_{i=1}^{n-1} \lambda_{i} z_{i}^{2}\right)^{2} d z^{\prime}+\circ\left(\epsilon^{2}+\delta^{2}\right)
\end{aligned}
$$

where we have used the fact that $\sum_{i=1}^{n-1} \lambda_{i}=0$, and the radial symmetry of $u_{1}$ to conclude that one of the integrals is zero. The second term in the expansion for $d$ is easy:

$$
\begin{aligned}
& \frac{c}{2} \int_{B_{\rho_{0} / \epsilon}^{n-1}} u_{1}^{\frac{2(n-1)}{n-2}}\left(z^{\prime}, \frac{f\left(\epsilon z^{\prime}\right)}{\epsilon}\right)\left(\frac{\epsilon^{2}}{3} \sum_{i, j=1}^{n-1} R_{n i n j} z_{i} z_{j}+\sum_{i=1}^{n-1} \epsilon^{2} \lambda_{i}^{2} z_{i}^{2}\right) d z^{\prime} \\
& =\frac{c \epsilon^{2}}{2} \int_{B_{\rho_{0} / \epsilon}^{n-1}} u_{1}^{\frac{2(n-1)}{n-2}}\left(z^{\prime}, 0\right)\left(\frac{1}{3} \sum_{i, j=1}^{n-1} R_{n i n j} z_{i} z_{j}+\sum_{i=1}^{n-1} \lambda_{i}^{2} z_{i}^{2}\right) d z^{\prime}+\circ\left(\epsilon^{2}+\delta^{2}\right) \\
& =\frac{c \epsilon^{2}}{2} \int_{\mathbf{R}^{n-1}} u_{1}^{\frac{2(n-1)}{n-2}}\left(z^{\prime}, 0\right)\left(\frac{1}{3} \sum_{i, j=1}^{n-1} R_{n i n j} z_{i} z_{j}+\sum_{i=1}^{n-1} \lambda_{i}^{2} z_{i}^{2}\right) d z^{\prime}+\circ\left(\epsilon^{2}+\delta^{2}\right) \\
& =\frac{c \epsilon^{2}}{2} \int_{\mathbf{R}^{n-1}} u_{1}^{\frac{2(n-1)}{n-2}}\left(z^{\prime}, 0\right)\left(\frac{1}{3} \sum_{i=1}^{n-1} R_{n i n i} z_{i}^{2}+\sum_{i=1}^{n-1} \lambda_{i}^{2} z_{i}^{2}\right) d z^{\prime}+\circ\left(\epsilon^{2}+\delta^{2}\right) \\
& =\frac{c \epsilon^{2}}{2} \int_{\mathbf{R}^{n-1}} u_{1}^{\frac{2(n-1)}{n-2}}\left(z^{\prime}, 0\right)\left(\frac{1}{3} R_{n n} z_{1}^{2}+\left(\sum_{i=1}^{n-1} \lambda_{i}^{2}\right) z_{1}^{2}\right) d z^{\prime}+\circ\left(\epsilon^{2}+\delta^{2}\right) \\
& =\frac{c \epsilon^{2}}{2}\left(\sum_{i=1}^{n-1} \lambda_{i}^{2}\right) \int_{\mathbf{R}^{n-1}} u_{1}^{\frac{2(n-1)}{n-2}}\left(z^{\prime}, 0\right) z_{1}^{2} d z^{\prime}+\circ\left(\epsilon^{2}+\delta^{2}\right) .
\end{aligned}
$$

In the above we have used the symmetry of $u_{1}$ and $R_{n n}=0$. Next we calculate the third term in the expansion for $d$.

$$
\begin{aligned}
& \frac{2(n-1) c}{(n-2)} \delta \int_{B_{\rho_{0} / \epsilon}^{n-1}} u_{1}^{\frac{n}{n-2}}\left(z^{\prime}, \frac{f\left(\epsilon z^{\prime}\right)}{\epsilon}\right) \phi\left(z^{\prime}, \frac{f\left(\epsilon z^{\prime}\right)}{\epsilon}\right) d z^{\prime} \\
& =\frac{2(n-1) c}{(n-2)} \delta \int_{B_{\rho_{0} / \epsilon}^{n-1}}\left\{u_{1}^{\frac{n}{n-2}}\left(z^{\prime}, 0\right) \phi\left(z^{\prime}, 0\right)\right.
\end{aligned}
$$




$$
\begin{aligned}
& \left.+\left.\frac{\partial}{\partial z_{n}}\left[u_{1}\left(z^{\prime}, z_{n}\right)^{\frac{n}{n-2}} \phi\left(z^{\prime}, z_{n}\right)\right]\right|_{z_{n}=0}\left[\frac{f\left(\epsilon z^{\prime}\right)}{\epsilon}\right]\right\} d z^{\prime}+\circ\left(\epsilon^{2}+\delta^{2}\right) \\
= & \frac{2(n-1) c}{(n-2)} \delta \int_{\mathbf{R}^{n-1}} u_{1}^{\frac{n}{n-2}}\left(z^{\prime}, 0\right) \phi\left(z^{\prime}, 0\right) d z^{\prime} \\
& +\left.\frac{(n-1) c}{(n-2)} \delta \epsilon \sum_{i=1}^{n-1} \int_{\mathbf{R}^{n-1}} \frac{\partial}{\partial z_{n}}\left[u_{1}\left(z^{\prime}, z_{n}\right)^{\frac{n}{n-2}} \phi\left(z^{\prime}, z_{n}\right)\right]\right|_{z_{n}=0}\left(\lambda_{i} z_{i}^{2}\right) \\
& +\circ\left(\epsilon^{2}+\delta^{2}\right) .
\end{aligned}
$$

The last term in the expansion for $d$ is

$$
\begin{aligned}
& \frac{(n-1) n c}{(n-2)^{2}} \delta^{2} \int_{B_{\rho_{0} / \epsilon}^{n-1}} u_{1}^{\frac{2}{n-2}}\left(z^{\prime}, \frac{f\left(\epsilon z^{\prime}\right)}{\epsilon}\right) \phi^{2}\left(z^{\prime}, \frac{f\left(\epsilon z^{\prime}\right)}{\epsilon}\right) d z^{\prime} \\
& \quad=\frac{(n-1) n c}{(n-2)^{2}} \delta^{2} \int_{\mathbf{R}^{n-1}} u_{1}^{\frac{2}{n-2}}\left(z^{\prime}, 0\right) \phi^{2}\left(z^{\prime}, 0\right) d z^{\prime}+\circ\left(\epsilon^{2}+\delta^{2}\right) .
\end{aligned}
$$

Putting these together, we have

$$
\begin{aligned}
d= & d_{0}+\left.\frac{c \epsilon^{2}}{8} \int_{\mathbf{R}^{n-1}} \frac{\partial^{2}}{\partial z_{n}^{2}}\left[u_{1}\left(z^{\prime}, z_{n}\right)^{\frac{2(n-1)}{n-2}}\right]\right|_{z_{n}=0}\left(\sum_{i=1}^{n-1} \lambda_{i} z_{i}^{2}\right)^{2} d z^{\prime} \\
& +\frac{c \epsilon^{2}}{2} \int_{\mathbf{R}^{n-1}} u_{1}^{\frac{2(n-1)}{n-2}}\left(z^{\prime}, 0\right)\left(\sum_{i=1}^{n-1} \lambda_{i}^{2} z_{i}^{2}\right) d z^{\prime} \\
& +\frac{2(n-1) c}{(n-2)} \delta \int_{\mathbf{R}^{n-1}} u_{1}^{\frac{n}{n-2}}\left(z^{\prime}, 0\right) \phi\left(z^{\prime}, 0\right) d z^{\prime} \\
& +\left.\frac{(n-1) c}{(n-2)} \delta \epsilon \sum_{i=1}^{n-1} \int_{\mathbf{R}^{n-1}} \frac{\partial}{\partial z_{n}}\left[u_{1}\left(z^{\prime}, z_{n}\right)^{\frac{n}{n-2}} \phi\left(z^{\prime}, z_{n}\right)\right]\right|_{z_{n}=0}\left(\lambda_{i} z_{i}^{2}\right) \\
& +\frac{(n-1) n c}{(n-2)^{2}} \delta^{2} \int_{\mathbf{R}^{n-1}} u_{1}^{\frac{2}{n-2}}\left(z^{\prime}, 0\right) \phi^{2}\left(z^{\prime}, 0\right) d z^{\prime}+\circ\left(\epsilon^{2}+\delta^{2}\right) .
\end{aligned}
$$

Thus

$$
\begin{aligned}
& D_{0}=\frac{2(n-1) c}{(n-2)} \int_{\mathbf{R}^{n-1}} u_{1}^{\frac{n}{n-2}}\left(z^{\prime}, 0\right) \phi\left(z^{\prime}, 0\right) d z^{\prime} \\
& D_{1}=\left.\frac{(n-1) c}{(n-2)} \sum_{i=1}^{n-1} \lambda_{i} \int_{\mathbf{R}^{n-1}} \frac{\partial}{\partial z_{n}}\left[u_{1}\left(z^{\prime}, z_{n}\right)^{\frac{n}{n-2}} \phi\left(z^{\prime}, z_{n}\right)\right]\right|_{z_{n}=0} z_{i}^{2} d z^{\prime} \\
& D_{2}=\frac{(n-1) n c}{(n-2)^{2}} \int_{\mathbf{R}^{n-1}} u_{1}^{\frac{2}{n-2}}\left(z^{\prime}, 0\right) \phi^{2}\left(z^{\prime}, 0\right) d z^{\prime}
\end{aligned}
$$




$$
\begin{aligned}
D_{3}= & \frac{c}{2} \sum_{i=1}^{n-1} \lambda_{i}^{2} \int_{\mathbf{R}^{n-1}} u_{1}^{\frac{2(n-1)}{n-2}}\left(z^{\prime}, 0\right) z_{i}^{2} d z^{\prime} \\
& +\left.\frac{c}{8} \int_{\mathbf{R}^{n-1}} \frac{\partial^{2}}{\partial z_{n}^{2}}\left[u_{1}\left(z^{\prime}, z_{n}\right)^{\frac{2(n-1)}{n-2}}\right]\right|_{z_{n}=0}\left(\sum_{i=1}^{n-1} \lambda_{i} z_{i}^{2}\right)^{2} d z^{\prime} .
\end{aligned}
$$

Recall that

$$
\max _{0<t<\infty} I(t u)=S_{c}+Q_{0} \delta+Q_{1} \epsilon \delta+Q_{2} \delta^{2}+Q_{3} \epsilon^{2}+\circ\left(\epsilon^{2}+\delta^{2}\right),
$$

where

$$
\begin{aligned}
Q_{0}= & \frac{1}{2}\left(A_{0}-(n-2)^{2} B_{0}-\frac{n-2}{n-1} D_{0}\right), \\
Q_{1}= & \frac{1}{2}\left(A_{1}-(n-2)^{2} B_{1}-\frac{n-2}{n-1} D_{1}\right), \\
Q_{2}= & \frac{1}{2}\left\{A_{2}-(n-2)^{2} B_{2}-\frac{n-2}{n-1} D_{2}\right. \\
& \left.+\frac{n-2}{2 a_{0}+2 n(n-2) b_{0}}\left[A_{0}-n(n-2) B_{0}-D_{0}\right]^{2}\right\}, \\
Q_{3}= & \frac{1}{2}\left(A_{3}-(n-2)^{2} B_{3}-\frac{n-2}{n-1} D_{3}\right) .
\end{aligned}
$$

We evaluate the $Q_{i}^{\prime} \mathrm{s}$ in terms of $\phi$, using the above calculations.

$$
Q_{0}=\int_{\mathbf{R}_{+}^{n}} \nabla u_{1} \nabla \phi-n(n-2) \int_{\mathbf{R}_{+}^{n}} u_{1}^{\frac{n+2}{n-2}} \phi-c \int_{\mathbf{R}^{n-1}} u_{1}^{\frac{n}{n-2}} \phi=0,
$$

and

$$
\begin{gathered}
Q_{1}=\frac{1}{2} \sum_{i=1}^{n-1} \lambda_{i} \int_{\mathbf{R}^{n-1}}\left\{-\nabla u_{1}\left(z^{\prime}, 0\right) \cdot \nabla \phi\left(z^{\prime}, 0\right) z_{i}^{2}+n(n-2) u_{1}^{\frac{n+2}{n-2}}\left(z^{\prime}, 0\right) \phi\left(z^{\prime}, 0\right) z_{i}^{2}\right. \\
\left.-\left.c \frac{\partial}{\partial z_{n}}\left[u_{1}\left(z^{\prime}, z_{n}\right)^{\frac{n}{n-2}} \phi\left(z^{\prime}, z_{n}\right)\right]\right|_{z_{n}=0} z_{i}^{2}\right\} d z^{\prime} \\
=\frac{1}{2} \sum_{i=1}^{n-1} \lambda_{i} \int_{\mathbf{R}^{n-1}}\left\{-\nabla^{\prime} u_{1}\left(z^{\prime}, 0\right) \cdot \nabla^{\prime} \phi\left(z^{\prime}, 0\right)+n(n-2) u_{1}^{\frac{n+2}{n-2}}\left(z^{\prime}, 0\right) \phi\left(z^{\prime}, 0\right)\right. \\
\left.-c \frac{n}{n-2} u_{1}^{\frac{2}{n-2}} \frac{\partial u_{1}}{\partial z_{n}} \phi\left(z^{\prime}, 0\right)\right\} z_{i}^{2}
\end{gathered}
$$




$$
\begin{aligned}
& =\frac{1}{2} \sum_{i=1}^{n-1} \lambda_{i} \int_{\mathbf{R}^{n-1}}\left\{-\nabla^{\prime} u_{1}\left(z^{\prime}, 0\right) \cdot \nabla^{\prime} \phi\left(z^{\prime}, 0\right)\right. \\
& \left.\quad+n(n-2)\left(1+T_{c}^{2}\right) u_{1}^{\frac{n+2}{n-2}}\left(z^{\prime}, 0\right) \phi\left(z^{\prime}, 0\right)\right\} z_{i}^{2}
\end{aligned}
$$

where we have used the boundary condition satisfied by $u_{1}$ and $\nabla^{\prime} u_{1}\left(z^{\prime}, 0\right)$ to denote $\left(\frac{\partial u_{1}}{\partial z_{1}}\left(z^{\prime}, 0\right), \cdots, \frac{\partial u_{1}}{\partial z_{n-1}}\left(z^{\prime}, 0\right)\right)$. Integrating by parts in the first integral above, we obtain

$$
\begin{aligned}
Q_{1}=\sum_{i=1}^{n-1} \frac{\lambda_{i}}{2} \int_{\mathbf{R}^{n-1}}\{( & \left.\sum_{j=1}^{n-1} \partial_{j j} u_{1}\left(z^{\prime}, 0\right)\right) \phi\left(z^{\prime}, 0\right) z_{i}^{2}+\nabla^{\prime} u_{1}\left(z^{\prime}, 0\right) \cdot \nabla^{\prime} z_{i}^{2} \phi\left(z^{\prime}, 0\right) \\
& \left.+n(n-2)\left(1+T_{c}^{2}\right) u_{1}^{\frac{n+2}{n-2}}\left(z^{\prime}, 0\right) \phi\left(z^{\prime}, 0\right) z_{i}^{2}\right\} \\
=\sum_{i=1}^{n-1} \frac{\lambda_{i}}{2} \int_{\mathbf{R}^{n-1}}\{ & -(n-2)\left[n\left(1+T_{c}^{2}\right)-\left(1+\left|z^{\prime}\right|^{2}+T_{c}^{2}\right)\right] u_{1}^{\frac{n+2}{n-2}}\left(z^{\prime}, 0\right) \\
& -2(n-2)\left(1+\left|z^{\prime}\right|^{2}+T_{c}^{2}\right) u_{1}^{\frac{n+2}{n-2}}\left(z^{\prime}, 0\right) \\
& \left.+n(n-2)\left(1+T_{c}^{2}\right) u_{1}^{\frac{n+2}{n-2}}\left(z^{\prime}, 0\right)\right\} \phi\left(z^{\prime}, 0\right) z_{i}^{2}
\end{aligned}
$$

Next

$$
\begin{aligned}
& Q_{2}=\frac{1}{2}\left\{\int_{\mathbf{R}_{+}^{n}}|\nabla \phi|^{2}-n(n+2) \int_{\mathbf{R}_{+}^{n}} u_{1}^{\frac{4}{n-2}} \phi^{2}+n T_{c} \int_{\mathbf{R}^{n-1}} u_{1}^{\frac{2}{n-2}}\left(z^{\prime}, 0\right) \phi^{2}\left(z^{\prime}, 0\right)\right. \\
& +\frac{n-2}{2 a_{0}+2 n(n-2) b_{0}}\left[2 \int_{\mathbf{R}_{+}^{n}} \nabla u_{1} \cdot \nabla \phi-2 n^{2} \int_{\mathbf{R}_{+}^{n}} u_{1}^{\frac{n+2}{n-2}} \phi\right. \\
& \left.\left.-\frac{2(n-1) c}{n-2} \int_{\mathbf{R}^{n-1}} u_{1}^{\frac{n}{n-2}}\left(z^{\prime}, 0\right) \phi\left(z^{\prime}, 0\right)\right]^{2}\right\} \\
& =\frac{1}{2}\left\{\int_{\mathbf{R}_{+}^{n}}|\nabla \phi|^{2}-n(n+2) \int_{\mathbf{R}_{+}^{n}} u_{1}^{\frac{4}{n-2}} \phi^{2}+n T_{c} \int_{\mathbf{R}^{n-1}} u_{1}^{\frac{2}{n-2}}\left(z^{\prime}, 0\right) \phi^{2}\left(z^{\prime}, 0\right)\right.
\end{aligned}
$$




$$
\begin{aligned}
& +\frac{n-2}{2 a_{0}+2 n(n-2) b_{0}}\left[-4 n \int_{\mathbf{R}_{+}^{n}} u_{1}^{\frac{n+2}{n-2}} \phi\right. \\
& \left.\left.+2 T_{c} \int_{\mathbf{R}^{n-1}} u_{1}^{\frac{n}{n-2}}\left(z^{\prime}, 0\right) \phi\left(z^{\prime}, 0\right)\right]^{2}\right\} .
\end{aligned}
$$

Finally

$$
\begin{aligned}
Q_{3}= & \frac{1}{2}\left\{-\frac{(n-2)^{2} T_{c}}{4} \int_{\mathbf{R}^{n-1}} \frac{(n-1)\left(\left|z^{\prime}\right|^{2}+T_{c}^{2}\right)-1}{\left(1+\left|z^{\prime}\right|^{2}+T_{c}^{2}\right)^{n+1}}\left(\sum_{i=1}^{n-1} \lambda_{i} z_{i}^{2}\right)^{2}\right. \\
& +\frac{n(n-2)^{2} T_{c}}{4} \int_{\mathbf{R}^{n-1}} \frac{\left(\sum_{i=1}^{n-1} \lambda_{i} z_{i}^{2}\right)^{2}}{\left(1+\left|z^{\prime}\right|^{2}+T_{c}^{2}\right)^{n+1}} d z^{\prime} \\
& -\frac{c(n-2)}{2(n-1)} \sum_{i=1}^{n-1} \lambda_{i}^{2} \int_{\mathbf{R}^{n-1}} u_{1}^{\frac{2(n-1)}{n-2}}\left(z^{\prime}, 0\right) z_{i}^{2} d z^{\prime} \\
& \left.-\left.\frac{c(n-2)}{8(n-1)} \int_{\mathbf{R}^{n-1}} \frac{\partial^{2}}{\partial z_{n}^{2}}\left[u_{1}\left(z^{\prime}, z_{n}\right)^{\frac{2(n-1)}{n-2}}\right]\right|_{z_{n}=0}\left(\sum_{i=1}^{n-1} \lambda_{i} z_{i}^{2}\right)^{2} d z^{\prime}\right\} \\
= & \frac{(n-2)^{2} T_{c}}{8} \int_{\mathbf{R}^{n-1}} \frac{n\left(1+T_{c}^{2}-\left|z^{\prime}\right|^{2}\right)}{\left(1+\left|z^{\prime}\right|^{2}+T_{c}^{2}\right)^{n+1}}\left(\sum_{i=1}^{n-1} \lambda_{i} z_{i}^{2}\right)^{2} \\
& -\frac{c(n-2)}{4(n-1)} \sum_{i=1}^{n-1} \lambda_{i}^{2} \int_{\mathbf{R}^{n-1}} u_{1}^{\frac{2(n-1)}{n-2}}\left(z^{\prime}, 0\right) z_{i}^{2} d z^{\prime} \\
= & \frac{(n-2)^{2} T_{c}}{8} \int_{\mathbf{R}^{n-1}} \frac{n\left(1+T_{c}^{2}-\left|z^{\prime}\right|^{2}\right)}{\left(1+\left|z^{\prime}\right|^{2}+T_{c}^{2}\right)^{n+1}}\left(\sum_{i=1}^{n-1} \lambda_{i} z_{i}^{2}\right)^{2} \\
& +\frac{(n-2)^{2} T_{c}}{4(n-1)} \int_{\mathbf{R}^{n-1}} \frac{\sum_{i=1}^{n-1} \lambda_{i}^{2} z_{i}^{2}}{\left(1+\left|z^{\prime}\right|^{2}+T_{c}^{2}\right)^{n-1}} .
\end{aligned}
$$

Writing the integrals in polar coordinates, and using the following elementary relations(the proof of which will be sketched in Appendix C)

$$
\begin{gathered}
\int_{0}^{\infty} \frac{r^{n+2}}{\left(1+r^{2}\right)^{n+1}} d r=\frac{n-3}{2 n} \int_{0}^{\infty} \frac{r^{n+2}}{\left(1+r^{2}\right)^{n}} d r, \text { for } n \geq 4 \\
\int_{0}^{\infty} \frac{r^{n}}{\left(1+r^{2}\right)^{n-1}} d r=2 \frac{n-1}{n+1} \int_{0}^{\infty} \frac{r^{n+2}}{\left(1+r^{2}\right)^{n}} d r, \text { for } n \geq 4 \\
\int_{\mathbf{S}^{n-2}} \xi_{1}^{4} d \xi=3 \int_{\mathbf{S}^{n-2}} \xi_{1}^{2} \xi_{2}^{2} d \xi=\frac{3}{n+1} \int_{\mathbf{S}^{n-2}} \xi_{1}^{2} d \xi=\frac{3 \sigma_{n-2}}{n^{2}-1}
\end{gathered}
$$


where $\sigma_{n-2}$ denotes the area of the standard sphere $\mathbf{S}^{n-2}, Q_{3}$ can be simplified as

$$
\begin{aligned}
Q_{3}= & \frac{n(n-2)^{2} T_{c}}{8}\left(1+T_{c}^{2}\right)^{\frac{3-n}{2}}\left(\int_{0}^{\infty} \frac{\left(1-r^{2}\right) r^{n+2}}{\left(1+r^{2}\right)^{n+1}} d r\right)\left(\int_{\mathbf{S}^{n-2}}\left(\sum_{i=1}^{n-1} \lambda_{i} \xi_{i}^{2}\right)^{2} d \xi\right) \\
& +\frac{(n-2)^{2} T_{c}}{4(n-1)}\left(1+T_{c}^{2}\right)^{\frac{3-n}{2}}\left(\int_{0}^{\infty} \frac{r^{n}}{\left(1+r^{2}\right)^{n-1}} d r\right)\left(\int_{\mathbf{S}^{n-2}} \sum_{i=1}^{n-1} \lambda_{i}^{2} \xi_{i}^{2} d \xi\right) \\
= & -\frac{\sigma_{n-2}(n-2)^{2} T_{c}}{8(n-1)^{2}}\left(1+T_{c}^{2}\right)^{\frac{3-n}{2}}\left(\sum_{i=1}^{n-1} \lambda_{i}^{2}\right)\left(\int_{0}^{\infty} \frac{r^{n}}{\left(1+r^{2}\right)^{n-1}} d r\right) \\
= & -q_{n} T_{c}\left(1+T_{c}^{2}\right)^{\frac{3-n}{2}} \sum_{i=1}^{n-1} \lambda_{i}^{2},
\end{aligned}
$$

here, $q_{n}$ denotes $\frac{\sigma_{n-2}(n-2)^{2}}{8(n-1)^{2}}\left(\int_{0}^{\infty} \frac{r^{n}}{\left(1+r^{2}\right)^{n-1}} d r\right)$. In the above, we have also used $\sum_{i=1}^{n-1} \lambda_{i}=0$ to obtain the relation

$$
\int_{\mathbf{S}^{n-2}}\left(\sum_{i=1}^{n-1} \lambda_{i} \xi_{i}^{2}\right)^{2} d \xi=2 \sum_{i=1}^{n-1} \lambda_{i}^{2} \int_{\mathbf{S}^{n-2}} \xi_{1}^{2} \xi_{2}^{2} d \xi
$$

We note that $Q_{3}$ is a constant depending only on $n, T_{c}$, and $\sum_{i=1}^{n-1} \lambda_{i}^{2}$. In particular, it is independent of $\phi$.

We would like to choose $\phi$ and $\epsilon \geq 0, \delta \geq 0$ small such that

$$
\max _{0<t<\infty} I(t u)<S_{c}
$$

which would lead to $I_{m p}<S_{c}$. It is clear from (2.3) that, in order for (2.10) to hold, it suffices to find a $\phi$ such that

$$
Q_{1}^{2}-4 Q_{2} Q_{3}>0 \text {. }
$$

From the expression of $Q_{3}$, it is clear that if $c \leq 0$, then $Q_{3} \leq 0$ and (2.11) can be satisfied easily. This proves the existence of a solution of (1.1) in the case $c \leq 0$. So we are only left to deal with the case of $c>0$. Since $Q_{3}$ is independent of $\phi, Q_{1}$ is a linear functional of $\phi$, and $Q_{2}$ is a quadratic functional of $\phi$, the verification of (2.11) in this case leads to an eigenvalue problem on $\mathbf{R}_{+}^{n}$, which may have independent interest. We will formulate and study this eigenvalue problem in the next section. Before we leave this section, we summarize our results of this section as 
Proposition 2.1. For $c \leq 0$, there exists a solution of (1.1). For $c>0$, if

$$
4 Q_{3}\left(\inf _{Q_{1}(\phi) \neq 0} \frac{Q_{2}(\phi)}{Q_{1}(\phi)^{2}}\right)<1
$$

then (1.1) has a solution.

Remark 2.1. We remark that, although we required $\phi$ to have compact support in evaluating the $Q_{i}$ 's, there is no need to restrict $\phi$ to have compact support in the extremal problem in Proposition 2.1. For, if (2.12) holds, a density argument can easily produce a $\phi$ with compact support satisfying (2.11). The precise space for $\phi$ is spelled out in the next section.

\section{A related eigenvalue problem on spherical caps.}

Because of the geometric invariance properties of the conformal Laplace operator, it is more transparent to translate the expressions for $Q_{1}, Q_{2}$ onto the round sphere. This is done as follows. Let $\Pi$ be the stereographic projection from the unit sphere in $\mathbf{R}^{n+1}$ centered at $\left(0, \cdots, 0, T_{c}, 0\right)$ onto the hyperplane $\xi_{n+1}=0$. More specifically, let $\left(\xi_{1}, \cdots, \xi_{n+1}\right)$ be the coordinates of $\mathbf{R}^{n+1}$ taking $\left(0, \cdots, 0, T_{c}, 0\right)$ as its origin and $\left(z_{1}, \cdots, z_{n}\right)$ be the coordinates of $\mathbf{R}^{n}$, which is identified with the hyperplane $\xi_{n+1}=0$. We take the unit sphere to be

$$
\mathbf{S}^{n}=\left\{\left(\xi_{1}, \cdots, \xi_{n+1}\right) \in \mathbf{R}^{n+1} \mid \xi_{1}^{2}+\cdots+\xi_{n}^{2}+\xi_{n+1}^{2}<1\right\} .
$$

Then, under the transformation $\Pi:\left(\xi_{1}, \cdots, \xi_{n+1}\right) \rightarrow\left(z_{1}, \cdots, z_{n}\right)$, we have

$$
\left\{\begin{aligned}
\xi_{i} & =\frac{2 z_{i}}{1+\left|z^{\prime}\right|^{2}+\left|z_{n}-T_{c}\right|^{2}}, 1 \leq i \leq n-1, \\
\xi_{n} & =\frac{2\left(z_{n}-T_{c}\right)}{1+\left|z^{\prime}\right|^{2}+\left|z_{n}-T_{c}\right|^{2}} \\
\xi_{n+1} & =\frac{\left|z^{\prime}\right|^{2}+\left|z_{n}-T_{c}\right|^{2}-1}{1+\left|z^{\prime}\right|^{2}+\left|z_{n}-T_{c}\right|^{2}}
\end{aligned}\right.
$$

Let $\Sigma=\Pi^{-1}\left(\mathbf{R}_{+}^{n}\right)$. It is a spherical cap on $\mathbf{S}^{n}$. For a function $\phi(z)$ defined on $\mathbf{R}_{+}^{n}$, we define a function $\Phi(\xi)$ on $\Sigma$ by

$$
\phi(z)=\Phi(\xi)\left(\frac{2}{1+\left|z^{\prime}\right|^{2}+\left|z_{n}-T_{c}\right|^{2}}\right)^{\frac{n-2}{2}}
$$


Note that the standard metric on $\Sigma$ is given by

$$
d s_{\text {sphere }}^{2}=\left(\frac{2}{1+\left|z^{\prime}\right|^{2}+\left|z_{n}-T_{c}\right|^{2}}\right)^{2}|d z|^{2} .
$$

The conformal Laplace operators $\left(L_{g}, B_{g}\right)$ enjoy the following conformal invariance property:

$$
\begin{aligned}
& L_{u^{\frac{4}{n-2}} g}\left(u^{-1} \phi\right)=u^{-\frac{n+2}{n-2}} L_{g}(\phi), \\
& B_{u^{\frac{4}{n-2}} g}\left(u^{-1} \phi\right)=u^{-\frac{n}{n-2}} B_{g}(\phi) .
\end{aligned}
$$

Using (3.3), we can compute the mean curvature of $\partial \Sigma$ to be

$$
\begin{aligned}
& -\frac{2}{n-2}\left(\frac{2}{1+\left|z^{\prime}\right|^{2}+\left|z_{n}-T_{c}\right|^{2}}\right)^{-\frac{n}{2}} \\
& \qquad\left.\frac{\partial}{\partial z_{n}}\right|_{z_{n}=0}\left\{\left(\frac{2}{1+\left|z^{\prime}\right|^{2}+\left|z_{n}-T_{c}\right|^{2}}\right)^{\frac{n-2}{2}}\right\}=-T_{c},
\end{aligned}
$$

and

$$
\begin{aligned}
\int_{\mathbf{R}_{+}^{n}}|\nabla \phi|^{2} & =-\int_{\mathbf{R}_{+}^{n}} \phi \Delta \phi+\int_{\partial \mathbf{R}_{+}^{n}} \phi \frac{\partial \phi}{\partial \nu} \\
& =-\int_{\mathbf{R}_{+}^{n}} \phi L_{|d z|^{2}}(\phi)+\int_{\partial \mathbf{R}_{+}^{n}} \phi B_{|d z|^{2}}(\phi) \\
& =\int_{\Sigma}\left[|\nabla \Phi|^{2}+\frac{n(n-2)}{4} \Phi^{2}\right]-\frac{n-2}{2} T_{c} \int_{\partial \Sigma} \Phi^{2} .
\end{aligned}
$$

Using (3.1), it is elementary to conclude that the linear functional $Q_{1}$ of $\phi$ given in (2.4) satisfies

$$
\begin{aligned}
Q_{1} & =-\sum_{i=1}^{n-1} \frac{(n-2) \lambda_{i}}{2^{\frac{n+2}{2}}} \int_{\partial \Sigma} \Phi(\xi) z_{i}^{2} \\
& =\int_{\partial \Sigma} f(\xi) \Phi(\xi)
\end{aligned}
$$

where $f(\xi)=-\sum_{i=1}^{n-1} \frac{(n-2) \lambda_{i}}{2^{\frac{n+2}{2}}} z_{i}^{2}$. In the following, we may abuse notation to write $Q_{1}(\Phi)$ for $Q_{1}$ to indicate its linear dependence on $\Phi$. Using (3.1) again, we find, for $u_{1}$ as given in (1.3),

$$
\int_{\mathbf{R}_{+}^{n}} u_{1}^{\frac{4}{n-2}} \phi^{2}=\frac{1}{4} \int_{\Sigma} \Phi^{2}
$$




$$
\begin{aligned}
& \int_{\partial \mathbf{R}_{+}^{n}} u_{1}^{\frac{2}{n-2}} \phi^{2}=\frac{1}{2} \int_{\partial \Sigma} \Phi^{2} \\
& \int_{\mathbf{R}_{+}^{n}} u_{1}^{\frac{n+2}{n-2}} \phi=\frac{1}{2^{\frac{n+2}{2}}} \int_{\Sigma} \Phi \\
& \int_{\partial \mathbf{R}_{+}^{n}} u_{1}^{\frac{n}{n-2}} \phi=\frac{1}{2^{n / 2}} \int_{\partial \Sigma} \Phi .
\end{aligned}
$$

Putting these together, we find that the quadratic form $Q_{2}$ of $\phi$ given in (??) satisfies

$$
\begin{aligned}
Q_{2}= & \frac{1}{2}\left\{\int_{\Sigma}\left(|\nabla \Phi|^{2}-n \Phi^{2}\right)+T_{c} \int_{\partial \Sigma} \Phi^{2}\right\} \\
& +\frac{n-2}{2^{n}\left[a_{0}+n(n-2) b_{0}\right]}\left\{T_{c} \int_{\partial \Sigma} \Phi-n \int_{\Sigma} \Phi\right\}^{2} .
\end{aligned}
$$

Again, we will write $Q_{2}(\Phi, \Phi)$ for $Q_{2}$ above to indicate its quadratic dependence on $\Phi$. We also use $Q_{2}(\Phi, \Psi)$ to denote the bilinear form obtained from symmetrization from the quadratic form $Q_{2}(\Phi, \Phi)$, i.e.,

$$
Q_{2}(\Phi, \Psi)=\frac{1}{4}\left[Q_{2}(\Phi+\Psi, \Phi+\Psi)-Q_{2}(\Phi-\Psi, \Phi-\Psi)\right] .
$$

Let $|\Sigma|,|\partial \Sigma|$ denote the volumes of $\Sigma$ and $\partial \Sigma$, respectively, with respect to the standard metric on $\mathbf{S}^{n}$. From the metric relation (3.2), it is elementary to check that

$$
\begin{aligned}
|\Sigma| & =\int_{\mathbf{R}_{+}^{n}}\left(\frac{2}{1+\left|z^{\prime}\right|^{2}+\left|z_{n}-T_{c}\right|^{2}}\right)^{n}=2^{n} \int_{\mathbf{R}_{+}^{n}} u_{1}^{\frac{2 n}{n-2}}=2^{n} b_{0}, \\
c|\partial \Sigma| & =c \int_{\partial \mathbf{R}_{+}^{n}}\left(\frac{2}{1+\left|z^{\prime}\right|^{2}+\left|z_{n}-T_{c}\right|^{2}}\right)^{n-1}=2^{n-1} c \int_{\partial \mathbf{R}_{+}^{n}} u_{1}^{\frac{2(n-1)}{n-2}}=2^{n-1} d_{0} .
\end{aligned}
$$

Using $c=-(n-2) T_{c}$ and (1.4), we easily obtain

$$
a_{0}+n(n-2) b_{0}=\frac{n-2}{2^{n-1}}\left(n|\Sigma|-T_{c}|\partial \Sigma|\right) .
$$

Define a linear operator $\mathcal{M}$ by

$$
\mathcal{M}(\Phi)=-\frac{T_{c} \int_{\partial \Sigma} \Phi-n \int_{\Sigma} \Phi}{T_{c}|\partial \Sigma|-n|\Sigma|}
$$

Note that $\mathcal{M}(1)=-1$. Define $\hat{\Phi}=\Phi+\mathcal{M}(\Phi)$. Then $\mathcal{M}(\hat{\Phi})=0$, and it is routine to check that

$$
Q_{2}(\Phi, \Phi)=\frac{1}{2}\left\{\int_{\Sigma}\left(|\nabla \Phi|^{2}-n \Phi^{2}\right)+T_{c} \int_{\partial \Sigma} \Phi^{2}\right\}+\frac{n|\Sigma|-T_{c}|\partial \Sigma|}{2} \mathcal{M}(\Phi)^{2}
$$




$$
\begin{aligned}
& =\frac{1}{2}\left\{\int_{\Sigma}\left(|\nabla \hat{\Phi}|^{2}-n \hat{\Phi}^{2}\right)+T_{c} \int_{\partial \Sigma} \hat{\Phi}^{2}\right\} \\
& =Q_{2}(\hat{\Phi}, \hat{\Phi}) .
\end{aligned}
$$

Because of $\sum_{i=1}^{n-1} \lambda_{i}=0$ and the coordinate symmetry, it is also easy to see that

$$
Q_{1}(\Phi)=Q_{1}(\hat{\Phi})
$$

Therefore we may work on the space $X=\left\{\Phi \in H^{1}(\Sigma): \mathcal{M}(\Phi)=0\right\}$ and drop the ${ }^{\wedge}$ on $\Phi$. Define, for $\lambda_{1}, \cdots, \lambda_{n-1} \in \mathbf{R}$, with $\sum_{i=1}^{n-1} \lambda_{i}=0$,

$$
\begin{aligned}
Q\left(n, c, \lambda_{1}, \cdots, \lambda_{n-1}\right) & =\inf _{\Phi \in H^{1}(\Sigma), Q_{1}(\Phi) \neq 0} \frac{Q_{2}(\Phi, \Phi)}{Q_{1}^{2}(\Phi)} \\
& =\inf _{\Phi \in X, Q_{1}(\Phi) \neq 0} \frac{Q_{2}(\Phi, \Phi)}{Q_{1}^{2}(\Phi)} .
\end{aligned}
$$

We first state

Proposition 3.1. $Q\left(n, c, \lambda_{1}, \cdots, \lambda_{n-1}\right)>0$. Furthermore, a minimizer $\Phi \in X$ of (3.4) exists, and $\Phi$ satisfies

$$
\left\{\begin{array}{l}
\Delta \Phi+n \Phi=0 \\
\partial_{\nu} \Phi+T_{c} \Phi=\mu f
\end{array}\right.
$$

with some Lagrange multiplier $\mu \neq 0$. Here $\nu$ denotes the unit outward normal of $\partial \Sigma$.

We will provide an elementary proof of Proposition 3.1 later. For now we remark that, if $\Phi$ is as in Proposition 3.1, then $\Phi / \mu \in X$ is also a minimizer of $Q\left(n, c, \lambda_{1} \cdots, \lambda_{n-1}\right)$. It satisfies (3.5) with $f$ replacing $\mu f$. For convenience, we will use this normalization $\mu=1$ in (3.5).

The homogenous version of (3.5) is of relevance:

$$
\left\{\begin{array}{l}
\Delta \Phi+n \Phi=0 \\
\partial_{\nu} \Phi+T_{c} \Phi=0
\end{array}\right.
$$

Integrating both sides of the first equation of (3.6) over $\Sigma$ and using the boundary condition in the second equation, we find that any solution of (3.6) is in $X$. We observe that the kernel of the quadratic form $Q_{2}$ in $H^{1}(\Sigma)$ consists of linear combinations of constants and solutions of (3.6). This can be easily seen by writing

$$
Q_{2}(\Phi, \Psi)=\frac{1}{2}\left\{\int_{\Sigma}[-\Delta \Phi-n(\Phi+\mathcal{M}(\Phi))] \Psi\right.
$$




$$
\left.+\int_{\partial \Sigma}\left[\partial_{\nu} \Phi+T_{c}(\Phi+\mathcal{M}(\Phi))\right] \Psi\right\}
$$

and noting that if $\Phi$ is in the kernel of $Q_{2}$, then $\Phi+\mathcal{M}(\Phi)$ is a solution of (3.6), thus $\Phi$, module a constant $\mathcal{M}(\Phi)$, is a solution of (3.6). Conversely, by noting that any solution of (3.6) is in $X$ and that $1+\mathcal{M}(1)=0$, it is obvious that the sum of any solution of (3.6) and any constant is in the kernel of $Q_{2}$. It will be shown below that, if we choose the center of $\Sigma$ as the north pole and choose corresponding Euclidean coordinates $\left(\zeta_{1}, \cdots, \zeta_{n+1}\right)$, then the restrictions of $\left\{\zeta_{1}, \cdots, \zeta_{n}\right\}$ to $\Sigma$ form a basis of the space of solutions of (3.6). Consequently, $\operatorname{Ker} Q_{2}:=\left\{\Phi \in H^{1}(\Sigma) \mid Q_{2}(\Phi, \Psi)=0, \forall \Psi \in\right.$ $\left.H^{1}(\Sigma)\right\}=\operatorname{span}\left\{1, \zeta_{1}, \zeta_{2}, \cdots, \zeta_{n}\right\}$. More generally, we will consider the eigenvalue problem

$$
\begin{cases}\Delta \Phi+n \Phi & =0, \\ \partial_{\nu} \Phi+T_{c} \Phi & =\mu \Phi .\end{cases}
$$

We summarize the relevant results concerning (3.7) in

Proposition 3.2. In the case of $T_{c}<0$, the eigenvalues of (3.7) have the distribution $\left\{\mu_{0}<0=\mu_{1}<\mu_{2} \cdots\right\}$, with $\mu_{0}=T_{c}+\frac{1}{T_{c}}$, and $\lim _{i \rightarrow \infty} \mu_{i}=\infty$. We can choose a complete set of eigenfunctions, $\Phi_{0}^{(1)}, \cdots, \Phi_{0}^{\left(k_{0}\right)}, \Phi_{1}^{(1)}, \cdots, \Phi_{1}^{\left(k_{1}\right)}, \Phi_{2}^{(1)}, \cdots$, so that their restrictions to $\partial \Sigma$ form an orthonormal basis of $L^{2}(\partial \Sigma)$. Here $k_{i}$ denotes the multiplicity of the eigenvalue $\mu_{i}$. Furthermore, $k_{0}=1$, and the eigenspace associated with $\mu_{0}$ is spanned by the restriction of $\zeta_{n+1}$ to $\Sigma ; k_{1}=n$, and the eigenspace associated with $\mu_{1}$ is spanned by the restrictions of $\zeta_{1}, \cdots, \zeta_{n}$ to $\Sigma$.

Remark 3.1. In the case of $T_{c} \geq 0$, the statements in Proposition 3.2, module obvious modifications, continue to hold. In particular, in the case of $T_{c}=0$, a modification is that the restrictions of the eigenfunctions to $\partial \Sigma$ span the $L^{2}$ orthogonal complement of constants in $L^{2}(\partial \Sigma)$. The proof is also essentially the same as the proof of Proposition 3.2, which we will give after we first use the set up here to simplify the condition in Proposition 2.1. In section 4 we will also give explicit forms of eigenfunctions associated with $\mu_{2}$ and $\mu_{3}$, which are needed for explicit verification of the condition in Proposition 2.1.

For now we remark that, due to Proposition 3.2, we could choose $\Phi_{0}^{(1)}$ to be a constant multiple of $\zeta_{n+1}$. Expand $f=\sum_{i \geq 0,1 \leq j \leq k_{i}} f_{j}^{(i)} \Phi_{i}^{(j)}$. Then $f_{j}^{(i)}=\int_{\partial \Sigma} f \Phi_{i}^{(j)}$. Since the restriction of $\zeta_{n+1}$ to $\partial \Sigma$ is a constant and 
$\int_{\partial \Sigma} f=0$ due to $\sum_{i=1}^{n-1} \lambda_{i}=0$ and the symmetry of $\partial \Sigma$, so $f_{1}^{(0)}=0$. Let $\Phi$ be a solution of (3.5) with $\mu=1$. Then, multiplying the first equation of (3.5) by $\Phi$ and integrating by parts, we obtain

$$
Q_{2}(\Phi, \Phi)=\frac{1}{2} Q_{1}(\Phi)
$$

Thus

$$
Q\left(n, c, \lambda_{1}, \cdots, \lambda_{n-1}\right)=\frac{1}{2 Q_{1}(\Phi)} .
$$

Multiplying $\Phi_{i}^{(j)}$ to the first equation in (3.5) and integrating by parts, we obtain

$$
\int_{\partial \Sigma} f \Phi_{i}^{(j)}=\mu_{i} \int_{\partial \Sigma} \Phi \Phi_{i}^{(j)}
$$

Thus

$$
\begin{aligned}
\int_{\partial \Sigma} f \Phi & =\sum_{i \geq 1,1 \leq j \leq k_{i}} f_{j}^{(i)} \int_{\partial \Sigma} \Phi \Phi_{i}^{(j)} \\
& =\sum_{\mu_{i}>0,1 \leq j \leq k_{i}} \frac{\left|f_{j}^{(i)}\right|^{2}}{\mu_{i}} .
\end{aligned}
$$

Therefore, the condition in Proposition 2.1 is equivalent to

$$
\sum_{\mu_{i}>0,1 \leq j \leq k_{i}} \frac{\left|f_{j}^{(i)}\right|^{2}}{\mu_{i}}>2 Q_{3} .
$$

We now give the proof of Proposition 3.2.

Proof of Proposition 3.2. We first derive the formulas for the eigenvalues of (3.7). Let $(r, \theta)$ be the geodesic polar coordinates on $\Sigma$ centered at the center of $\Sigma$, where $r$ is the geodesic distance on $\Sigma$ from the center. We remark that $\cos r=-T_{c} / \sqrt{1+T_{c}^{2}}$ on $\partial \Sigma$. We can write

$$
d s_{\text {sphere }}^{2}=d r^{2}+\sin ^{2} r d \theta^{2}, \quad \theta \in \mathbf{S}^{n-1} .
$$

Thus

$$
\Delta_{\text {sphere }} \Phi=\Phi_{r r}+(n-1) \cot r \Phi_{r}+\frac{1}{\sin ^{2} r} \Delta_{\theta} \Phi .
$$

Using the method of separation of variables, we write $\Phi(r, \theta)=E(r) \Psi(\theta)$. Then, from the first equation of (3.7), we obtain

$$
\left\{\begin{array}{l}
E_{r r}+(n-1) \cot r E_{r}+n E+\frac{\lambda}{\sin ^{2} r} E=0, \\
\Delta_{\theta} \Psi=\lambda \Psi
\end{array}\right.
$$


From the second equation above, it is standard that $\lambda=\lambda_{k}=-k(n+k-2)$, for $k=0,1,2, \cdots$. The second equation of (3.7) becomes

$$
\mu=T_{c}+\left.\frac{E_{r}(r)}{E(r)}\right|_{\partial \Sigma} .
$$

We make a change of variable $\zeta=\cos r$ and set $F(\zeta)=E(r)$. We remark that, since we are considering only the case of $T_{c}<0$, the relevant range for $\zeta$ in Proposition 3.2 is $0<\zeta \leq 1$. However, most of what follows holds for $-1<\zeta<1$. Using

$$
\begin{aligned}
E_{r} & =-\sin r F^{\prime}(\zeta), \\
E_{r r} & =-\cos r F^{\prime}(\zeta)+\sin ^{2} r F^{\prime \prime}(\zeta),
\end{aligned}
$$

the equation for $E$ transforms into

$$
\left(1-\zeta^{2}\right) F^{\prime \prime}(\zeta)-n \zeta F^{\prime}(\zeta)+n F(\zeta)-\frac{k(n+k-2)}{1-\zeta^{2}} F(\zeta)=0
$$

$\zeta= \pm 1$ are the singular points of (3.9). The indicies of (3.9) at $\zeta= \pm 1$ can be easily found to be $\alpha=k / 2$, or $\alpha=-(n+k-2) / 2$. Since we look for $F$ which is regular near $\zeta=1$, the latter index is discarded. Set $F(\zeta)=\left(1-\zeta^{2}\right)^{k / 2} G(\zeta)$. From (3.9), we obtain

$$
\left(1-\zeta^{2}\right) G^{\prime \prime}(\zeta)-(2 k+n) \zeta G^{\prime}(\zeta)+(1-k)(k+n) G(\zeta)=0 .
$$

Denote a solution of $\left(E V_{k}\right)$ by $G_{k}$. Differentiating both sides of $\left(E V_{k}\right)$, we find that $G_{k}^{\prime}(\zeta)$ satisfies $\left(E V_{k+1}\right)$. We will use this relation to find the solutions of $\left(E V_{k}\right) .\left(E V_{1}\right)$ is the easiest to solve. Setting $k=1$ in $\left(E V_{k}\right)$, we obtain

$$
\left(1-\zeta^{2}\right) G_{1}^{\prime \prime}(\zeta)-(2+n) \zeta G_{1}^{\prime}(\zeta)=0
$$

from which we easily obtain

$$
G_{1}^{\prime}(\zeta)=A\left(1-\zeta^{2}\right)^{-\frac{n+2}{2}}
$$

for some constant $A$. Thus

$$
G_{1}(\zeta)=A \int_{0}^{\zeta}\left(1-\eta^{2}\right)^{-\frac{n+2}{2}} d \eta+B
$$

for some constant $B$. Since we need a regular $F_{1}$, we set $A=0$ and $B=1$ to obtain

$$
F_{1}=\left(1-\zeta^{2}\right)^{\frac{1}{2}}
$$


$G_{1}^{\prime}$ is a singular solution of $\left(E V_{2}\right)$. However, using standard ODE theory, we find the general solution of $\left(E V_{2}\right)$ to be

$$
\left(1-\zeta^{2}\right)^{-\frac{n+2}{2}}\left\{A \int_{\zeta}^{1}\left(1-\eta^{2}\right)^{n / 2} d \eta+B\right\}
$$

for some constants $A$ and $B$. The obvious choice is to set

$$
G_{2}(\zeta)=\left(1-\zeta^{2}\right)^{-\frac{n+2}{2}} \int_{\zeta}^{1}\left(1-\eta^{2}\right)^{n / 2} d \eta
$$

Thus

$$
F_{2}(\zeta)=\left(1-\zeta^{2}\right)^{-n / 2} \int_{\zeta}^{1}\left(1-\eta^{2}\right)^{n / 2} d \eta
$$

Now it is obvious that we should set, for $k \geq 2$,

$$
G_{k}(\zeta)=\frac{d^{k-2} G_{2}(\zeta)}{d \zeta^{k-2}}
$$

Therefore,

$$
F_{k}(\zeta)=\left(1-\zeta^{2}\right)^{k / 2} \frac{d^{k-2} G_{2}(\zeta)}{d \zeta^{k-2}}
$$

We remark that the $G_{k}$ defined above will not be identically zero and thus we have obtained nontrivial solutions for $\left(E V_{k}\right)$ which is smooth near $\zeta=1$ for any $k \geq 2$. This is because $G_{2}(\zeta)$ is smooth in $-1<\zeta \leq 1$ and is not a polynomial in $\zeta$, which can be seen either from the explicit expression of $G_{2}$ or from the following argument: if $G_{2}$ were a polynomial of order $l$, then, $G_{l+2}$ is a nonzero constant which should be a solution of $\left(E V_{l+2}\right)$. That is impossible by direct inspection.

We will also need the following properties of $G_{k}$ :

$$
\text { for } k \geq 2, \quad G_{k}(\zeta) \neq 0, \quad \text { and } \quad G_{k}^{\prime}(\zeta) / G_{k}(\zeta)<0,
$$

in the range $-1<\zeta<1$. We now establish these properties. Suppose the contrary, i.e., $G_{k}\left(\zeta_{0}\right)=0$, or $G_{k}^{\prime}\left(\zeta_{0}\right) / G_{k}\left(\zeta_{0}\right) \geq 0$ at some $-1<\zeta_{0}<1$. In the first situation, we may assume that $G_{k}^{\prime}\left(\zeta_{0}\right)>0$, and in the second situation, we may assume that $G_{k}\left(\zeta_{0}\right)>0$. We note from $\left(E V_{k}\right)$ that $G_{k}^{\prime \prime}(\zeta)>0$ whenever $G_{k}^{\prime}(\zeta)=0$ and $G_{k}(\zeta)$ is positive. Using this observation, it is easy to prove that $G_{k}(\zeta)$ can not have a positive local maxima in $\zeta_{0}<\zeta<1$ and therefore $G_{k}^{\prime}(\zeta)$ and $G_{k}(\zeta)$ will be positive for $\zeta_{0}<\zeta \leq 1$. Since $G_{k}$ is smooth near $\zeta=1$, sending $\zeta$ to 1 in $\left(E V_{k}\right)$ would lead to a contradiction. 
Next we derive the formula for $G_{0}$. Since $G_{0}^{\prime}$ is a solution of $\left(E V_{1}\right)$, it is easy to figure out the choice for $G_{0}$ should be $G_{0}(\zeta)=\zeta$, which implies that $F_{0}(\zeta)=\zeta$.

At this point we introduce $\mu_{k}$ to denote the eigenvalue of (3.7) associated with $\lambda_{k}$. Using the fact that $\zeta=-T_{c} / \sqrt{1+T_{c}^{2}}$ on $\partial \Sigma$, we have the formula

$$
\begin{aligned}
\mu_{k} & =T_{c}+F_{k}^{-1}(\zeta) \frac{d F_{k}(\zeta)}{d \zeta} \frac{d \zeta}{d r} \\
& =T_{c}-\frac{d F_{k}(\zeta) / d \zeta}{\sqrt{1+T_{c}^{2}} F_{k}(\zeta)}
\end{aligned}
$$

with $\zeta$ evaluated at $-T_{c} / \sqrt{1+T_{c}^{2}}$. Therefore,

$$
\mu_{0}=T_{c}+\frac{1}{T_{c}}
$$

and

$$
\mu_{1}=T_{c}-T_{c}=0 .
$$

From (3.11), we also deduce

$$
\mu_{k}=(1-k) T_{c}-\frac{G_{k}^{\prime}(\zeta)}{\sqrt{1+T_{c}^{2}} G_{k}(\zeta)}
$$

with $\zeta$ evaluated at $-T_{c} / \sqrt{1+T_{c}^{2}}$.

We next prove that $\mu_{k}<\mu_{l}$ for $1 \leq k<l$. Since we have proved that $F_{k}, F_{l} \neq 0$ in the range $-1<\zeta<1$, we may assume $F_{k}, F_{l}>0$ for $-1<\zeta<1$. From (3.9), we deduce easily

$$
\begin{aligned}
{\left[\left(F_{k}^{\prime} F_{l}-F_{k} F_{l}^{\prime}\right)\left(1-\zeta^{2}\right)^{\frac{n}{2}}\right]^{\prime} } & \\
& +[l(n+l-2)-k(n+k-2)]\left(1-\zeta^{2}\right)^{\frac{n}{2}-2} F_{k} F_{l}=0
\end{aligned}
$$

which gives, for $k<l$,

$$
\left[\left(F_{k}^{\prime} F_{l}-F_{k} F_{l}^{\prime}\right)\left(1-\zeta^{2}\right)^{\frac{n}{2}}\right]^{\prime}<0, \quad \text { for } \quad-1<\zeta<1,
$$

from which we obtain $F_{k}^{\prime}(\zeta) / F_{k}(\zeta)>F_{l}^{\prime}(\zeta) / F_{l}(\zeta)$. With (3.11), we conclude that $\mu_{k}<\mu_{l}$.

We now observe that, since $L^{2}(\partial \Sigma)$ has a complete basis in terms of the eigenfunctions $\Psi_{k}^{(l)}$ of the Laplace operator $\Delta_{\mathbf{S}^{n-1}}$, the corresponding $\Phi_{k}^{(l)}(r, \theta)=E_{k}(r) \Psi_{k}^{(l)}(\theta)$, when restricted to $\partial \Sigma$, naturally form a basis of 
$L^{2}(\partial \Sigma)$ (recall that $F_{k}(\zeta) \neq 0$ for all $k \geq 0$ in the range $0<\zeta<1$ ). In particular, the corresponding eigenfunctions associated with $\mu_{0}$ are spanned by

$$
\Phi_{0}^{(1)}(r, \theta)=\cos r
$$

and those associated with $\mu_{1}$ are spanned by

$$
\Phi_{1}^{(l)}(r, \theta)=\sin r \Psi_{1}^{(l)}(\theta), l=1, \cdots, n,
$$

where $\left\{\Psi_{1}^{(1)}(\theta), \Psi_{1}^{(2)}(\theta), \cdots, \Psi_{1}^{(n)}(\theta)\right\}$ can be taken as the coordinate functions of $\mathbf{S}^{n-1}$ and forms a basis of the space of eigenfunctions of $\Delta_{\mathbf{S}^{n-1}}$ associated with $\lambda_{1}=1-n$. We remark that $\Phi_{1}^{(1)}(r, \theta), \Phi_{1}^{(2)}(r, \theta), \cdots, \Phi_{1}^{(n)}(r, \theta)$ are precisely the coordinate functions $\zeta_{1}, \zeta_{2}, \cdots, \zeta_{n}$, and $\Phi_{0}$ is $\zeta_{n+1}$, when we rotate the Euclidean coordate $\xi_{1}, \cdots, \xi_{n+1}$ so that the $\zeta_{n+1}$-axis passes through the center of $\Sigma$. We also denote by $\left\{\Phi_{k}^{(l)}\right\}$ a basis of eigenfunctions associated with $\mu_{k}$ obtained as above through separation of variables, and remark that, for notational simplicity, we do not normalize the $\Phi_{k}^{(l)}$ 's to have unit $L^{2}(\partial \Sigma)$ norm at this moment.

To complete the proof of Proposition 3.2, we only need to show that the eigenvalues $\left\{\mu_{k}\right\}$ we have found above are the only eigenvalues of (3.7), and that the eigenfunctions obtained for each $\mu_{k}$ using seperation of variables span the eigenspace associated with $\mu_{k}$. Let $\Phi$ be an eigenfunction of (3.7) associated with some $\mu$. Multiplying the first equation of (3.7) by $\Phi_{k}^{(l)}$ and integrating by parts over $\Sigma$, we obtain

$$
\left(\mu-\mu_{k}\right) \int_{\partial \Sigma} \Phi \Phi_{k}^{(l)}=0 .
$$

Writing $\Phi=\sum_{k, l} a_{k}^{l} \Phi_{k}^{(l)}$ on $\partial \Sigma$. It follows that $\mu=\mu_{i}$ for some $i$, and $a_{k}^{l}=0$ for any $k \neq i$ and arbitrary $l$. Then $\Phi-\sum_{l} a_{i}^{l} \Phi_{i}^{(l)}$ satisfies the first equation in (3.7) and vanishes on $\partial \Sigma$ together with its normal derivative on $\partial \Sigma$. This implies that $\Phi-\sum_{l} a_{i}^{l} \Phi_{i}^{(l)} \equiv 0$ in $\Sigma$. Proposition 3.2 is thus established.

Before we proceed further, we also deduce a recursion formula for $\mu_{k}$, which may be of independent interest.

\section{Lemma 3.3.}

$$
\mu_{k+1}=(n+k) \frac{\left(1+T_{c}^{2}\right)(k-1)+T_{c} \mu_{k}}{T_{c}(k-1)+\mu_{k}}, \quad k \geq 2 .
$$


Furthermore, when $T_{c}<0$, we have the estimates

$$
-T_{c}(k-1)<\mu_{k}<\left(-T_{c}-\frac{1}{T_{c}}\right)(k-1), \quad k \geq 2 .
$$

Proof of Lemma 3.3. For $k \geq 2$, we divide both sides of $\left(E V_{k}\right)$ by $G_{k}^{\prime}$ to obtain

$$
\left(1-\zeta^{2}\right) \frac{G_{k}^{\prime \prime}(\zeta)}{G_{k}^{\prime}(\zeta)}-(2 k+n) \zeta+(1-k)(k+n) \frac{G_{k}(\zeta)}{G_{k}^{\prime}(\zeta)}=0 .
$$

Expressing $G_{k} / G_{k}^{\prime}$ in terms of $\mu_{k}$, and $G_{k}^{\prime \prime} / G_{k}^{\prime}$ in terms of $\mu_{k+1}$, and also noting $\zeta=\frac{-T_{c}}{\sqrt{1+T_{c}^{2}}}, 1-\zeta^{2}=\frac{1}{1+T_{c}^{2}}$, we have

$$
\frac{-k T_{c}-\mu_{k+1}}{\sqrt{1+T_{c}^{2}}}+(n+2 k) \frac{T_{c}}{\sqrt{1+T_{c}^{2}}}+\frac{(n+k)(1-k)}{\sqrt{1+T_{c}^{2}}\left[-T_{c}(k-1)-\mu_{k}\right]}=0,
$$

from which we deduce (3.13). The first inequality of (3.14) follows from (3.12) and (3.10). This inequality implies, in particular, that $\mu_{k}>0$, for $k \geq 2$. Using this in (3.13), we obtain the second inequality of (3.14).

Before providing the proof of Proposition 3.1, we first state another useful

Proposition 3.4. For all $\Phi \in H^{1}(\Sigma)$,

$$
Q_{2}(\Phi, \Phi) \geq 0
$$

Furthermore, $Q_{2}(\Phi, \Phi)=0$ iff $\Phi$ is a linear combination of $1, \zeta_{1}, \zeta_{2}, \cdots, \zeta_{n}$, and for some $\lambda>0, Q_{2}(\Phi, \Phi) \geq \lambda\|\Phi\|_{H^{1}(\Sigma)}^{2}, \forall \Phi \in\left(\operatorname{Ker} Q_{2}\right)^{\perp}$, where $\left(\operatorname{Ker} Q_{2}\right)^{\perp}$ is the orthogonal complement of $\operatorname{Ker} Q_{2}$ in $H^{1}(\Sigma)$ under $H^{1}$ inner product.

Proof of Proposition 3.4. We will provide a proof using the knowledge of the eigenvalues of (3.7). We first make the

Claim: $\min \left\{Q_{2}(\Phi, \Phi) \mid \Phi \in H^{1}(\Sigma), \int_{\partial \Sigma} \Phi^{2}=1\right\}$ is achieved.

Assuming the Claim for the moment, and letting $\Phi$ be a minimizer, then

$$
\begin{cases}\Delta \Phi+n(\Phi+\mathcal{M}(\Phi)) & =0, \\ \partial_{\nu} \Phi+T_{c}(\Phi+\mathcal{M}(\Phi)) & =\mu \Phi,\end{cases}
$$


where the Lagrange multipler $\mu$ is $\min \left\{Q_{2}(\Phi, \Phi) \mid \Phi \in H^{1}(\Sigma), \int_{\partial \Sigma} \Phi^{2}=1\right\}$. Integrating both sides of the first equation over $\Sigma$ and using the boundary condition in the second equation, we see easily that $\mu \int_{\partial \Sigma} \Phi=0$. If $\Phi$ is a non-zero constant on $\partial \Sigma$, then $\mu$ has to be zero and we are done. If $\Phi$ is not a constant, then $\hat{\Phi}=\Phi+\mathcal{M}(\Phi)$ is a nontrivial solution of

$$
\left\{\begin{array}{l}
\Delta \hat{\Phi}+n \hat{\Phi}=0 \\
\partial_{\nu} \hat{\Phi}+T_{c} \hat{\Phi}=\mu\left(\hat{\Phi}-\frac{1}{|\partial \Sigma|} \int_{\partial \Sigma} \hat{\Phi}\right) .
\end{array}\right.
$$

If $T_{c} \neq 0$, we multiply the first equation above by $\Phi_{0}$ and integrate by parts over $\Sigma$ to obtain $\mu_{0} \int_{\partial \Sigma} \Phi_{0} \hat{\Phi}=0$. Here we have used the observation that $\Phi_{0}$ is a non-zero constant on $\partial \Sigma$. Using this observation again, we conclude that $\int_{\partial \Sigma} \hat{\Phi}=0$. If $T_{c}=0$, then $\Phi_{0}=0$ on $\partial \Sigma$, and $\partial_{\nu} \Phi_{0}$ is a constant on $\partial \Sigma$. Multiplying the first equation above by $\Phi_{0}$ and integrating by parts over $\Sigma$, we obtain again $\int_{\partial \Sigma} \hat{\Phi}=0$. Going back to the equation satisfied by $\hat{\Phi}$ and recalling that $\hat{\Phi}$ is nontrivial in this situation, we conclude from Proposition 3.2 that $\mu=\mu_{i}$, and $\mu_{i} \geq 0$ because the only possible negative eigenvalue is $\mu_{0}$ when $T_{c}<0$ and non-trivial eigenfunctions associated with $\mu_{0}$ take non-zero constant values on $\partial \Sigma$, but we have just proved that $\int_{\partial \Sigma} \hat{\Phi}=0$. So we have proved that $\min _{\int_{\partial \Sigma} \Phi^{2}=1} Q_{2}(\Phi, \Phi)=\mu \geq 0$. In fact, it is equal to 0 because of the presence of the kernel of $Q_{2}$. This provides a proof for the first part of Proposition 3.4. The second part of Proposition 3.4 follows from our knowledge of solutions of (3.6).

Proof of the Claim. Let $\Phi_{i}$ be a minimizing sequence for $\min \left\{Q_{2}(\Phi, \Phi) \mid \Phi \in\right.$ $\left.H^{1}(\Sigma), \int_{\partial \Sigma} \Phi^{2}=1\right\}$. It will subconverge to a minimizer, provided that $\int_{\Sigma} \Phi_{i}^{2}$ stays bounded. Suppose, on the contrary, that $\int_{\Sigma} \Phi_{i}^{2} \rightarrow \infty$. Define $\tilde{\Phi}_{i}=\Phi_{i} /\left\|\Phi_{i}\right\|_{L^{2}(\Sigma)}$. Then, after passing to a subsequence, $\tilde{\Phi}_{i} \rightarrow \tilde{\Phi}_{\infty}$ weakly in $H^{1}(\Sigma)$, and $\tilde{\Phi}_{i} \rightarrow \tilde{\Phi}_{\infty}$ in $L^{2}(\Sigma)$, where $\tilde{\Phi}_{\infty}$ satisfies

$$
\begin{cases}Q_{2}\left(\tilde{\Phi}_{\infty}, \tilde{\Phi}_{\infty}\right) & \leq 0 \\ \left\|\tilde{\Phi}_{\infty}\right\|_{L^{2}(\Sigma)} & =1 \\ \left.\tilde{\Phi}_{\infty}\right|_{\partial \Sigma} & =0 .\end{cases}
$$

This implies, from the last boundary condition above and the expression of $Q_{2}$, that

$$
\int_{\Sigma}\left|\nabla \tilde{\Phi}_{\infty}\right|^{2}-n \tilde{\Phi}_{\infty}^{2}+\frac{n^{2}}{n|\Sigma|-T_{c}|\partial \Sigma|}\left(\int_{\Sigma} \tilde{\Phi}_{\infty}\right)^{2} \leq 0
$$


In the case $T_{c}<0,(3.16)$ implies that the first eigenvalue, $\lambda_{1}(\Sigma)$, of $\Delta_{\mathbf{S}^{n}}$ on $\Sigma$ with the zero Dirichlet boundary condition is less than or equal to $n$. On the other hand, the first eigenvalue, $\lambda_{1}\left(\mathbf{S}_{+}^{n}\right)$, of $\Delta_{\mathbf{S}^{n}}$ on the halfsphere $\mathbf{S}_{+}^{n}$ with the zero Dirichlet boundary condition is equal to $n$, and from the variational characterization for such eigenvalues, $\lambda_{1}(\Sigma)>\lambda_{1}\left(\mathbf{S}_{+}^{n}\right)$, since $\Sigma \subset \mathbf{S}_{+}^{n}$. This is a contradiction.

In the case $T_{c} \geq 0,(3.16)$, together with the obvious fact

$$
0<n|\Sigma|-T_{c}|\partial \Sigma|<n \sigma_{n}
$$

implies that

$$
\begin{aligned}
\int_{\mathbf{S}^{n}}\left|\nabla \tilde{\Phi}_{\infty}\right|^{2} & -n \int_{\mathbf{S}^{n}}\left(\tilde{\Phi}_{\infty}-\frac{1}{\sigma_{n}} \int_{\mathbf{S}^{n}} \tilde{\Phi}_{\infty}\right)^{2} \\
& =\int_{\Sigma}\left|\nabla \tilde{\Phi}_{\infty}\right|^{2}-n \tilde{\Phi}_{\infty}^{2}+\frac{n}{\sigma_{n}}\left(\int_{\Sigma} \tilde{\Phi}_{\infty}\right)^{2} \\
& \leq 0
\end{aligned}
$$

here we have extended $\tilde{\Phi}_{\infty}$ to be 0 on $\mathbf{S}^{n} \backslash \Sigma$ noting $\left.\tilde{\Phi}_{\infty}\right|_{\partial \Sigma}=0$ from (3.15). However, the first eigenvalue estimate on $\mathbf{S}^{n}$ says

$$
\int_{\mathbf{S}^{n}}|\nabla f|^{2}-n \int_{\mathbf{S}^{n}}\left(f-\frac{1}{\sigma_{n}} \int_{\mathbf{S}^{n}} f\right)^{2} \geq 0
$$

with equality iff $f$ is a first degree spherical harmonic. The extended $\tilde{\Phi}_{\infty}$, being equal to 0 on an open set, obviously can't be a first degree spherical harmonic. Thus we have reached a contradiction. The Claim thus holds in all cases.

Remark 3.2. (3.17) actually holds without the restriction of $T_{c} \geq 0$, so the proof of the Claim in that paragraph works for all cases. Separate proofs were given above to avoid a necessary (though simple) calculation to verify (3.17).

Proof of Proposition 3.1. Observe that, from the coordinate symmetry and the assumption $\sum_{1}^{n-1} \lambda_{i}=0$, we find $Q_{1}\left(\Phi-\Phi^{\prime}\right)=0$, if $\Phi-\Phi^{\prime} \in$ $\operatorname{Ker} Q_{2}$. Thus, for a minimizing sequence $\left\{\Phi_{i}\right\}$ for (3.4), we may assume that $\Phi_{i} \in\left(\operatorname{Ker} Q_{2}\right)^{\perp}$. We may also assume that $Q_{1}\left(\Phi_{i}\right)=1$ by scaling. From Proposition 3.4, $Q_{2}(\Phi, \Phi) \geq \lambda\|\Phi\|_{H^{1}(\Sigma)}^{2}$ for any $\Phi \in\left(\operatorname{Ker} Q_{2}\right)^{\perp}$. It follows 
easily from this that a subsequence of $\left\{\Phi_{i}\right\}$ weakly converges in $H^{1}(\Sigma)$ to a minimizer $\Phi$. It is routine to check that $\Phi$ satisfies the Euler-Lagrange equation (3.5). The $\mu$ in (3.5) can't be zero, otherwise, $\Phi$ is in the kernel of $Q_{2}$, which implies $Q_{1}(\Phi)=0$ in view of the observation above. This violates the constraint $Q_{1}(\Phi)=1$. The positivity of $Q\left(n, c, \lambda_{1}, \cdots, \lambda_{n-1}\right)$ is obvious now.

\section{The case of $c>0$..}

We will proceed to estimate $Q\left(n, c, \lambda_{1}, \cdots, \lambda_{n-1}\right)$. We first write down the precise transformation from $\xi$ to $\zeta$ :

$$
\begin{cases}\zeta_{i} & =\xi_{i}, \quad i=1, \cdots, n-1 \\ \zeta_{n} & =-\frac{T_{c} \xi_{n}+\xi_{n+1}}{\sqrt{1+T_{c}^{2}}} \\ \zeta_{n+1} & =\frac{\xi_{n}-T_{c} \xi_{n+1}}{\sqrt{1+T_{c}^{2}}}\end{cases}
$$

Therefore, on $\partial \Sigma$, we have

$$
z_{i}^{2}=\frac{\xi_{i}^{2}}{\left(1-\xi_{n+1}\right)^{2}}=\frac{\left(1+T_{c}^{2}\right)^{2} \zeta_{i}^{2}}{\left(1+\sqrt{1+T_{c}^{2}} \zeta_{n}\right)^{2}}, 1 \leq i \leq n-1 .
$$

We will make use of the eigenvalues $\mu_{2}$ and $\mu_{3}$. For this purpose, we first remark that a basis for the space of eigenfunctions of $\Delta_{\mathbf{S}^{n-1}}$ associated with $\lambda_{2}=-2 n$ can be taken as the restrictions to $\partial \Sigma$ of $\left\{\zeta_{j}^{2}-\frac{1}{n\left(1+T_{c}^{2}\right)}, j=\right.$ $\left.1, \cdots, n-1 ; \zeta_{i} \zeta_{j}, 1 \leq i<j \leq n\right\}$. The $\zeta_{i} \zeta_{j}$ 's are mutually orthogonal to each other, and are orthogonal to the $\zeta_{k}^{2}-\frac{1}{n\left(1+T_{c}^{2}\right)}$ 's, in $L^{2}(\partial \Sigma)$. However, the $\zeta_{k}^{2}-\frac{1}{n\left(1+T_{c}^{2}\right)}$ 's are not orthogonal to each other. Note, however, that in (3.8) all we need to compute is the square of the $L^{2}(\partial \Sigma)$ norm of the component $f^{(2)}$ of $f$ in $\operatorname{span}\left\{\zeta_{j}^{2}-\frac{1}{n\left(1+T_{c}^{2}\right)}, j=1, \cdots, n-1 ; \zeta_{i} \zeta_{j}, 1 \leq i<j \leq n\right\}$. For obvious symmetry reasons, the integrals on $\partial \Sigma$ of $z_{k}^{2}$ with $\zeta_{i} \zeta_{j}$ is zero, for $i \neq j$. So if we set $\Psi_{2}^{(j)}=\zeta_{j}^{2}-\frac{1}{n\left(1+T_{c}^{2}\right)}, j=1, \cdots, n$, we can write $f^{(2)}=\sum_{j=1}^{n-1} f_{j}^{(2)} \Psi_{2}^{(j)}$. A direct calculation, using (2.8), shows that

$$
\begin{aligned}
\int_{\partial \Sigma}\left|\Psi_{2}^{(j)}\right|^{2} & =\int_{\partial \Sigma}\left\{\zeta_{j}^{4}-\frac{2 \zeta_{j}^{2}}{n\left(1+T_{c}^{2}\right)}+\frac{1}{n^{2}\left(1+T_{c}^{2}\right)^{2}}\right\} \\
& =\frac{1}{\left(1+T_{c}^{2}\right)^{\frac{n+3}{2}}} \int_{\mathbf{S}^{n-1}}\left\{\theta_{j}^{4}-\frac{2 \theta_{j}^{2}}{n}+\frac{1}{n^{2}}\right\}
\end{aligned}
$$




$$
\begin{aligned}
\quad\left(\text { letting } \zeta=\frac{\theta}{\sqrt{1+T_{c}^{2}}}\right) \\
=\frac{1}{\left(1+T_{c}^{2}\right)^{\frac{n+3}{2}}}\left\{\frac{3 \sigma_{n-1}}{n(n+2)}-\frac{2 \sigma_{n-1}}{n^{2}}+\frac{\sigma_{n-1}}{n^{2}}\right\} \\
=\frac{2(n-1) \sigma_{n-1}}{n^{2}(n+2)\left(1+T_{c}^{2}\right)^{\frac{n+3}{2}}},
\end{aligned}
$$

which is independent of $j$ and will be denoted as $I_{2}$. A similar computation gives, for $i \neq j$,

$$
\begin{aligned}
\int_{\partial \Sigma} \Psi_{2}^{(i)} \Psi_{2}^{(j)} & =\int_{\partial \Sigma}\left\{\zeta_{i}^{2} \zeta_{j}^{2}-\frac{\zeta_{i}^{2}+\zeta_{j}^{2}}{n\left(1+T_{c}^{2}\right)}+\frac{1}{n^{2}\left(1+T_{c}^{2}\right)^{2}}\right\} \\
& =\frac{1}{\left(1+T_{c}^{2}\right)^{\frac{n+3}{2}}} \int_{\mathbf{S}^{n-1}}\left\{\theta_{i}^{2} \theta_{j}^{2}-\frac{\theta_{i}^{2}+\theta_{j}^{2}}{n}+\frac{1}{n^{2}}\right\} \\
& \quad\left(\text { letting } \zeta=\frac{\theta}{\sqrt{1+T_{c}^{2}}}\right) \\
& =\frac{1}{\left(1+T_{c}^{2}\right)^{\frac{n+3}{2}}}\left\{\frac{\sigma_{n-1}}{n(n+2)}-\frac{2 \sigma_{n-1}}{n^{2}}+\frac{\sigma_{n-1}}{n^{2}}\right\} \\
& =-\frac{2 \sigma_{n-1}}{n^{2}(n+2)\left(1+T_{c}^{2}\right)^{\frac{n+3}{2}}},
\end{aligned}
$$

which is also independent of $i, j$, and is actually equal to $-\frac{I_{2}}{n-1}$. Now

$$
\begin{aligned}
\int_{\partial \Sigma}\left|f^{(2)}\right|^{2} & =\sum_{j=1}^{n-1}\left|f_{j}^{(2)}\right|^{2} \int_{\partial \Sigma}\left|\Psi_{2}^{(j)}\right|^{2}+\sum_{i \neq j} f_{i}^{(2)} f_{j}^{(2)} \int_{\partial \Sigma} \Psi_{2}^{(i)} \Psi_{2}^{(j)} \\
& =\left(\sum_{j=1}^{n-1}\left|f_{j}^{(2)}\right|^{2}\right) I_{2}-\frac{I_{2}}{n-1}\left(\sum_{i \neq j} f_{i}^{(2)} f_{j}^{(2)}\right) \\
& =\frac{n I_{2}}{n-1}\left(\sum_{j=1}^{n-1}\left|f_{j}^{(2)}\right|^{2}\right)-\frac{I_{2}}{n-1}\left(\sum_{j=1}^{n-1} f_{j}^{(2)}\right)^{2} .
\end{aligned}
$$

The $f_{i}^{(2)}$ 's are determined by

$$
\sum_{i=1}^{n-1} f_{i}^{(2)} \int_{\partial \Sigma} \Psi_{2}^{(i)} \Psi_{2}^{(j)}=\int_{\partial \Sigma} f \Psi_{2}^{(j)}
$$


The coefficient matrix of this linear system of algebraic equations is

$$
\left[\begin{array}{ccccc}
I_{2} & -\frac{I_{2}}{n-1} & \ldots & \ldots & -\frac{I_{2}}{n-1} \\
-\frac{I_{2}}{n-1} & I_{2} & -\frac{I_{2}}{n-1} & \ldots & -\frac{I_{2}}{n-1} \\
\vdots & & & & \vdots \\
-\frac{I_{2}}{n-1} & \cdots & \cdots & -\frac{I_{2}}{n-1} & I_{2}
\end{array}\right]
$$

whose inverse is given as (it will be verified that $I_{2} \neq 0$ )

$$
\frac{(n-1)}{n I_{2}}\left[\begin{array}{cccc}
2 & 1 & \cdots & 1 \\
1 & 2 & \cdots & 1 \\
\vdots & \vdots & \vdots & \vdots \\
1 & 1 & \cdots & 2
\end{array}\right]
$$

Therefore

$$
f_{i}^{(2)}=\frac{(n-1)}{n I_{2}}\left\{\int_{\partial \Sigma} f \Psi_{2}^{(i)}+\sum_{j=1}^{n-1} \int_{\partial \Sigma} f \Psi_{2}^{(j)}\right\} .
$$

Observe that $\sum_{j=1}^{n-1} \Psi_{2}^{(j)}=-\Psi_{2}^{(n)}$. Using $\sum_{j=1}^{n-1} \lambda_{j}=0$ and coordinate symmetry, we find that

$$
\sum_{j=1}^{n-1} \int_{\partial \Sigma} f \Psi_{2}^{(j)}=0
$$

Thus

$$
f_{i}^{(2)}=\frac{(n-1)}{n I_{2}} \int_{\partial \Sigma} f \Psi_{2}^{(i)},
$$

and

$$
\sum_{i=1}^{n-1} f_{i}^{(2)}=0
$$

We now obtain a simplified expression for

$$
\begin{aligned}
\int_{\partial \Sigma}\left|f^{(2)}\right|^{2} & =\frac{n I_{2}}{n-1}\left(\sum_{j=1}^{n-1}\left|f_{j}^{(2)}\right|^{2}\right) \\
& =\frac{n-1}{n I_{2}} \sum_{j=1}^{n-1}\left(\int_{\partial \Sigma} f \Psi_{2}^{(j)}\right)^{2} .
\end{aligned}
$$

We next carry out a similar computation using the third eigenvalue of $\Delta_{\mathbf{S}^{n-1}}$. Let $\Psi_{3}^{(j)}=\left(\zeta_{j}^{2}-\frac{1}{(n+2)\left(1+T_{c}^{2}\right)}\right) \zeta_{n}, 1 \leq j<n$. Then $\Psi_{3}^{(j)}$ are eigenfunctions of 
The existence of conformal metrics with constant scalar curvature 847

$\Delta_{\mathbf{S}^{n-1}}$ corresponding to $\lambda_{3}=-3(n+1)$. Similar to the computation done for $\Psi_{2}^{(j)}$ 's, we denote by $f^{(3)}$ the component of $f$ in $\operatorname{span}\left\{\Psi_{3}^{(1)}, \Psi_{3}^{(2)}, \cdots, \Psi_{3}^{(n-1)}\right\}$, and write $f^{(3)}=\sum_{j=1}^{n-1} f_{j}^{(3)} \Psi_{3}^{(j)}$. We first compute, for $1 \leq j<n$,

$$
\begin{aligned}
\int_{\partial \Sigma}\left[\Psi_{3}^{(j)}\right]^{2} & =\int_{\partial \Sigma}\left(\zeta_{j}^{2}-\frac{1}{(n+2)\left(1+T_{c}^{2}\right)}\right)^{2} \zeta_{n}^{2} \\
& =\frac{1}{\left(1+T_{c}^{2}\right)^{\frac{n+5}{2}}} \int_{\mathbf{S}^{n-1}}\left\{\theta_{j}^{4}-\frac{2}{n+2} \theta_{j}^{2}+\frac{1}{(n+2)^{2}}\right\} \theta_{n}^{2} d \theta \\
& =\frac{1}{\left(1+T_{c}^{2}\right)^{\frac{n+5}{2}}}\left\{\frac{3 \sigma_{n-1}}{(n+4)(n+2) n}-\frac{\sigma_{n-1}}{(n+2)^{2} n}\right\} \\
& =\frac{2(n+1) \sigma_{n-1}}{(n+4)(n+2)^{2} n\left(1+T_{c}^{2}\right)^{\frac{n+5}{2}}},
\end{aligned}
$$

which is independent of $j$ and will be denoted as $I_{3}$. In the above, we have used,

$$
\int_{\mathbf{S}^{n-1}} \theta_{j}^{2} \theta_{n}^{2} d \theta=\frac{\sigma_{n-1}}{n(n+2)}
$$

and

$$
\int_{\mathbf{S}^{n-1}} \theta_{j}^{4} \theta_{n}^{2} d \theta=\frac{3 \sigma_{n-1}}{(n+4)(n+2) n} \quad \text { for } \quad j<n .
$$

We will also need, for $i \neq j<n$,

$$
\int_{\mathbf{S}^{n-1}} \theta_{i}^{2} \theta_{j}^{2} \theta_{n}^{2} d \theta=\frac{\sigma_{n-1}}{(n+4)(n+2) n} .
$$

(4.2) is just a version of (2.8). The derivation of (4.3) and (4.4) is similar to that of (2.8), and will be sketched in Appendix C. Using (4.4), for $i \neq j<n$,

$$
\begin{aligned}
\int_{\partial \Sigma} \Psi_{3}^{(i)} \Psi_{3}^{(j)}= & \int_{\partial \Sigma}\left\{\zeta_{i}^{2} \zeta_{j}^{2}-\frac{\zeta_{i}^{2}+\zeta_{j}^{2}}{(n+2)\left(1+T_{c}^{2}\right)}+\frac{1}{(n+2)^{2}\left(1+T_{c}^{2}\right)^{2}}\right\} \zeta_{n}^{2} \\
= & \frac{1}{\left(1+T_{c}^{2}\right)^{\frac{n+5}{2}}} \int_{\mathbf{S}^{n-1}}\left\{\theta_{i}^{2} \theta_{j}^{2}-\frac{\theta_{i}^{2}+\theta_{j}^{2}}{(n+2)}+\frac{1}{(n+2)^{2}}\right\} \theta_{n}^{2} \\
& \left(\operatorname{letting} \zeta=\frac{\theta}{\sqrt{1+T_{c}^{2}}}\right)
\end{aligned}
$$




$$
\begin{aligned}
& =\frac{1}{\left(1+T_{c}^{2}\right)^{\frac{n+5}{2}}}\left\{\frac{\sigma_{n-1}}{n(n+2)(n+4)}-\frac{2 \sigma_{n-1}}{n(n+2)^{2}}+\frac{\sigma_{n-1}}{n(n+2)^{2}}\right\} \\
& =-\frac{2 \sigma_{n-1}}{n(n+2)^{2}(n+4)\left(1+T_{c}^{2}\right)^{\frac{n+5}{2}}},
\end{aligned}
$$

which is also independent of $i, j$, and is actually equal to $-\frac{I_{3}}{n+1}$. Now

$$
\begin{aligned}
\int_{\partial \Sigma}\left|f^{(3)}\right|^{2} & =\sum_{j=1}^{n-1}\left|f_{j}^{(3)}\right|^{2} \int_{\partial \Sigma}\left|\Psi_{3}^{(j)}\right|^{2}+\sum_{i \neq j} f_{i}^{(3)} f_{j}^{(3)} \int_{\partial \Sigma} \Psi_{3}^{(i)} \Psi_{3}^{(j)} \\
& =\left(\sum_{j=1}^{n-1}\left|f_{j}^{(3)}\right|^{2}\right) I_{3}-\frac{I_{3}}{n+1}\left(\sum_{i \neq j} f_{i}^{(3)} f_{j}^{(3)}\right) \\
& =\frac{(n+2) I_{3}}{n+1}\left(\sum_{j=1}^{n-1}\left|f_{j}^{(3)}\right|^{2}\right)-\frac{I_{3}}{n+1}\left(\sum_{j=1}^{n-1} f_{j}^{(3)}\right)^{2}
\end{aligned}
$$

The $f_{i}^{(3)}$ 's are determined by

$$
\sum_{i=1}^{n-1} f_{i}^{(3)} \int_{\partial \Sigma} \Psi_{3}^{(i)} \Psi_{3}^{(j)}=\int_{\partial \Sigma} f \Psi_{3}^{(j)} .
$$

The coefficient matrix of this linear system of algebraic equations is

$$
\left[\begin{array}{ccccc}
I_{3} & -\frac{I_{3}}{n+1} & \cdots & \cdots & -\frac{I_{3}}{n+1} \\
-\frac{I_{3}}{n+1} & I_{3} & -\frac{I_{3}}{n+1} & \cdots & -\frac{I_{3}}{n+1} \\
\vdots & & & & \vdots \\
-\frac{I_{3}}{n+1} & \cdots & \cdots & -\frac{I_{3}}{n+1} & I_{3}
\end{array}\right]
$$

whose inverse is given as (it will be verified that $I_{3} \neq 0$ )

$$
\frac{(n+1)}{3(n+2) I_{3}}\left[\begin{array}{cccc}
4 & 1 & \cdots & 1 \\
1 & 4 & \cdots & 1 \\
\vdots & \vdots & \vdots & \vdots \\
1 & 1 & \cdots & 4
\end{array}\right]
$$

Therefore

$$
f_{i}^{(3)}=\frac{(n+1)}{3(n+2) I_{3}}\left\{3 \int_{\partial \Sigma} f \Psi_{3}^{(i)}+\sum_{j=1}^{n-1} \int_{\partial \Sigma} f \Psi_{3}^{(j)}\right\} .
$$


The existence of conformal metrics with constant scalar curvature $\quad 849$

Observe that $\sum_{j=1}^{n-1} \Psi_{3}^{(j)}=\left(\frac{3}{(n+2)\left(1+T_{c}^{2}\right)}-\zeta_{n}^{2}\right) \zeta_{n}$. Using $\sum_{j=1}^{n-1} \lambda_{j}=0$ and coordinate symmetry, we find that

$$
\sum_{j=1}^{n-1} \int_{\partial \Sigma} f \Psi_{3}^{(j)}=0
$$

Thus

$$
f_{i}^{(3)}=\frac{(n+1)}{(n+2) I_{3}} \int_{\partial \Sigma} f \Psi_{3}^{(i)}
$$

and

$$
\sum_{i=1}^{n-1} f_{i}^{(3)}=0 .
$$

We now obtain a simplified expression for

$$
\begin{aligned}
\int_{\partial \Sigma}\left|f^{(3)}\right|^{2} & =\frac{(n+2) I_{3}}{n+1}\left(\sum_{j=1}^{n-1}\left|f_{j}^{(3)}\right|^{2}\right) \\
& =\frac{n+1}{(n+2) I_{3}} \sum_{j=1}^{n-1}\left(\int_{\partial \Sigma} f \Psi_{3}^{(j)}\right)^{2} .
\end{aligned}
$$

We now proceed to evaluate the terms in the right hand sides of (4.1) and (4.5). We first compute, for $i \neq j<n$,

$$
\begin{aligned}
\int_{\partial \Sigma} z_{i}^{2} \Psi_{2}^{(j)} & \\
& =\int_{\partial \Sigma} \frac{\left(1+T_{c}^{2}\right)^{2} \zeta_{i}^{2}}{\left(1+\sqrt{1+T_{c}^{2}} \zeta_{n}\right)^{2}}\left(\zeta_{j}^{2}-\frac{1}{n\left(1+T_{c}^{2}\right)}\right) \\
& =\frac{1}{\left(1+T_{c}^{2}\right)^{\frac{n-1}{2}}} \int_{\mathbf{S}^{n-1}} \frac{\theta_{i}^{2}}{\left(1+\theta_{n}\right)^{2}}\left(\theta_{j}^{2}-\frac{1}{n}\right) \\
& =\frac{1}{\left(1+T_{c}^{2}\right)^{\frac{n-1}{2}}} \int_{-1}^{1} \frac{d \theta_{n}}{\sqrt{1-\theta_{n}^{2}}} \int_{\theta_{1}^{2}+\cdots+\theta_{n-1}^{2}=1-\theta_{n}^{2}} \frac{\theta_{i}^{2}}{\left(1+\theta_{n}\right)^{2}}\left(\theta_{j}^{2}-\frac{1}{n}\right) \\
& =\frac{1}{\left(1+T_{c}^{2}\right)^{\frac{n-1}{2}}} \int_{-1}^{1}\left(1-\theta_{n}^{2}\right)^{\frac{n-1}{2}}\left[\left(1-\theta_{n}^{2}\right) \frac{\sigma_{n-2}}{n^{2}-1}-\frac{\sigma_{n-2}}{n(n-1)}\right] \frac{d \theta_{n}}{\left(1+\theta_{n}\right)^{2}}
\end{aligned}
$$




$$
=-\frac{\sigma_{n-2}}{n\left(n^{2}-1\right)\left(1+T_{c}^{2}\right)^{\frac{n-1}{2}}} \int_{-1}^{1} \frac{\left(1-\theta_{n}^{2}\right)^{\frac{n-1}{2}}\left(1+n \theta_{n}^{2}\right) d \theta_{n}}{\left(1+\theta_{n}\right)^{2}} .
$$

Similarly,

$$
\begin{aligned}
& \int_{\partial \Sigma} z_{j}^{2} \Psi_{2}^{(j)} \\
&=\int_{\partial \Sigma} \frac{\left(1+T_{c}^{2}\right)^{2} \zeta_{j}^{2}}{\left(1+\sqrt{1+T_{c}^{2}} \zeta_{n}\right)^{2}}\left(\zeta_{j}^{2}-\frac{1}{n\left(1+T_{c}^{2}\right)}\right) \\
&=\frac{1}{\left(1+T_{c}^{2}\right)^{\frac{n-1}{2}}} \int_{\mathbf{S}^{n-1}} \frac{\theta_{j}^{2}}{\left(1+\theta_{n}\right)^{2}}\left(\theta_{j}^{2}-\frac{1}{n}\right) \quad\left(\operatorname{letting} \zeta=\frac{\theta}{\sqrt{1+T_{c}^{2}}}\right) \\
&=\frac{1}{\left(1+T_{c}^{2}\right)^{\frac{n-1}{2}}} \int_{-1}^{1} \frac{d \theta_{n}}{\sqrt{1-\theta_{n}^{2}}} \int_{\theta_{1}^{2}+\cdots+\theta_{n-1}^{2}=1-\theta_{n}^{2}} \frac{\theta_{j}^{2}}{\left(1+\theta_{n}\right)^{2}}\left(\theta_{j}^{2}-\frac{1}{n}\right) \\
&= \frac{1}{\left(1+T_{c}^{2}\right)^{\frac{n-1}{2}}} \int_{-1}^{1}\left(1-\theta_{n}^{2}\right)^{\frac{n-1}{2}}\left[\left(1-\theta_{n}^{2}\right) \frac{3 \sigma_{n-2}}{n^{2}-1}-\frac{\sigma_{n-2}}{n(n-1)}\right] \frac{d \theta_{n}}{\left(1+\theta_{n}\right)^{2}} \\
&=\frac{\sigma_{n-2}}{n\left(n^{2}-1\right)\left(1+T_{c}^{2}\right)^{\frac{n-1}{2}}} \int_{-1}^{1} \frac{\left(1-\theta_{n}^{2}\right)^{\frac{n-1}{2}}\left(2 n-1-3 n \theta_{n}^{2}\right) d \theta_{n}}{\left(1+\theta_{n}\right)^{2}}
\end{aligned}
$$

With these computations, we have

$$
\begin{aligned}
\int_{\partial \Sigma} & \left(\sum_{i=1}^{n-1} \lambda_{i} z_{i}^{2}\right) \Psi_{2}^{(j)} \\
= & \sum_{i \neq j} \lambda_{i} \int_{\partial \Sigma} z_{i}^{2} \Psi_{2}^{(j)}+\lambda_{j} \int_{\partial \Sigma} z_{j}^{2} \Psi_{2}^{(j)} \\
= & -\left(\sum_{i \neq j} \lambda_{i}\right) \frac{\sigma_{n-2}}{n\left(n^{2}-1\right)\left(1+T_{c}^{2}\right)^{\frac{n-1}{2}}} \int_{-1}^{1} \frac{\left(1-\theta_{n}^{2}\right)^{\frac{n-1}{2}}\left(1+n \theta_{n}^{2}\right) d \theta_{n}}{\left(1+\theta_{n}\right)^{2}} \\
& +\lambda_{j} \frac{\sigma_{n-2}}{n\left(n^{2}-1\right)\left(1+T_{c}^{2}\right)^{\frac{n-1}{2}}} \int_{-1}^{1} \frac{\left(1-\theta_{n}^{2}\right)^{\frac{n-1}{2}}\left(2 n-1-3 n \theta_{n}^{2}\right) d \theta_{n}}{\left(1+\theta_{n}\right)^{2}} \\
= & \frac{2 \sigma_{n-2} \lambda_{j}}{\left(n^{2}-1\right)\left(1+T_{c}^{2}\right)^{\frac{n-1}{2}}} \int_{-1}^{1} \frac{\left(1-\theta_{n}^{2}\right)^{\frac{n+1}{2}}}{\left(1+\theta_{n}\right)^{2}} d \theta_{n},
\end{aligned}
$$


where we used $\sum_{i=1}^{n-1} \lambda_{i}=0$ in the third line above. Thus

$$
\begin{aligned}
\int_{\partial \Sigma}\left|f^{(2)}\right|^{2}= & \sum_{j=1}^{n-1} \frac{(n-2)^{2}}{2^{n+2}}\left\{\frac{2 \sigma_{n-2} \lambda_{j}}{\left(n^{2}-1\right)\left(1+T_{c}^{2}\right)^{\frac{n-1}{2}}} \int_{-1}^{1} \frac{\left(1-\theta_{n}^{2}\right)^{\frac{n+1}{2}}}{\left(1+\theta_{n}\right)^{2}} d \theta_{n}\right\}^{2} \\
& \frac{n(n+2)\left(1+T_{c}^{2}\right)^{\frac{n+3}{2}}}{2 \sigma_{n-1}} \\
= & \left(\sum_{j=1}^{n-1} \lambda_{j}^{2}\right) \frac{n(n-2)^{2}(n+2) \sigma_{n-2}^{2}}{2^{n+1}(n-1)^{2}(n+1)^{2} \sigma_{n-1}}\left(1+T_{c}^{2}\right)^{-\frac{n-5}{2}} \\
& \left\{\int_{-1}^{1} \frac{\left(1-\theta_{n}^{2}\right)^{\frac{n+1}{2}}}{\left(1+\theta_{n}\right)^{2}} d \theta_{n}\right\}^{2} .
\end{aligned}
$$

To simplify this expression, we first observe

$$
\begin{aligned}
\int_{-1}^{1} \frac{\left(1-\theta_{n}^{2}\right)^{\frac{n+1}{2}}}{\left(1+\theta_{n}\right)^{2}} d \theta_{n} & =\int_{-1}^{1}\left(1+\theta_{n}\right)^{\frac{n-3}{2}}\left(1-\theta_{n}\right)^{\frac{n+1}{2}} d \theta \\
& \left.=2^{n} \int_{0}^{1} t^{\frac{n+1}{2}}(1-t)^{\frac{n-3}{2}} d t \quad \text { (letting } 1-\theta_{n}=2 t\right) \\
& =2^{n} B\left(\frac{n+3}{2}, \frac{n-1}{2}\right),
\end{aligned}
$$

where $B(p, q)=\int_{0}^{1} t^{p-1}(1-t)^{q-1} d t$ is the Beta function. Observe also that

$$
\begin{aligned}
\sigma_{n-1} & =\sigma_{n-2} \int_{-1}^{1}\left(1-\theta_{n}^{2}\right)^{\frac{n-3}{2}} d \theta_{n} \\
& =\sigma_{n-2} 2^{n-2} \int_{0}^{1} t^{\frac{n-3}{2}}(1-t)^{\frac{n-3}{2}} d t \\
& =2^{n-2} \sigma_{n-2} B\left(\frac{n-1}{2}, \frac{n-1}{2}\right) .
\end{aligned}
$$

Using these relations, we obtain

$$
\begin{aligned}
\int_{\partial \Sigma}\left|f^{(2)}\right|^{2}= & \left(\sum_{j=1}^{n-1} \lambda_{j}^{2}\right) \frac{n(n-2)^{2}(n+2) \sigma_{n-2}^{2}\left(1+T_{c}^{2}\right)^{-\frac{n-5}{2}}}{2^{n+1}(n-1)^{2}(n+1)^{2} 2^{n-2} \sigma_{n-2} B\left(\frac{n-1}{2}, \frac{n-1}{2}\right)} \\
& \left\{2^{n} B\left(\frac{n+3}{2}, \frac{n-1}{2}\right)\right\}^{2} .
\end{aligned}
$$


Using $B\left(\frac{n+3}{2}, \frac{n-1}{2}\right)=\frac{n+1}{4 n} B\left(\frac{n-1}{2}, \frac{n-1}{2}\right)$, we find

$$
\left\{B\left(\frac{n+3}{2}, \frac{n-1}{2}\right)\right\}^{2} / B\left(\frac{n-1}{2}, \frac{n-1}{2}\right)=\frac{(n+1)^{2}}{16 n^{2}} B\left(\frac{n-1}{2}, \frac{n-1}{2}\right) .
$$

Putting these together, we have

$$
\begin{aligned}
\int_{\partial \Sigma}\left|f^{(2)}\right|^{2}= & \left(\sum_{j=1}^{n-1} \lambda_{j}^{2}\right) \frac{2^{2 n} n(n-2)^{2}(n+1)^{2}(n+2) \sigma_{n-2}\left(1+T_{c}^{2}\right)^{-\frac{n-5}{2}}}{2^{2 n+3} n^{2}(n-1)^{2}(n+1)^{2}} \\
& B\left(\frac{n-1}{2}, \frac{n-1}{2}\right) \\
= & \left(\sum_{j=1}^{n-1} \lambda_{j}^{2}\right) \frac{(n-2)^{2}(n+2)\left(1+T_{c}^{2}\right)^{-\frac{n-5}{2}}}{2^{3} n(n-1)^{2}} \sigma_{n-2} B\left(\frac{n-1}{2}, \frac{n-1}{2}\right) \\
= & \left(\sum_{j=1}^{n-1} \lambda_{j}^{2}\right) \frac{(n-2)^{2}(n+2)\left(1+T_{c}^{2}\right)^{-\frac{n-5}{2}}}{2^{n+1} n(n-1)^{2}} \sigma_{n-1} .
\end{aligned}
$$

To evaluate the right hand side of (4.5), we first compute, for $i \neq j<n$,

$$
\begin{aligned}
\int_{\partial \Sigma} z_{i}^{2} \Psi_{3}^{(j)}=\int_{\partial \Sigma} \frac{\left(1+T_{c}^{2}\right)^{2} \zeta_{i}^{2}}{\left(1+\sqrt{1+T_{c}^{2}} \zeta_{n}\right)^{2}}\left(\zeta_{j}^{2}-\frac{1}{(n+2)\left(1+T_{c}^{2}\right)}\right) \zeta_{n} \\
=\int_{\mathbf{S}^{n-1}} \frac{\theta_{i}^{2}\left(\theta_{j}^{2}-\frac{1}{n+2}\right) \theta_{n}}{\left(1+T_{c}^{2}\right)^{n / 2}\left(1+\theta_{n}\right)^{2}} d \theta \quad\left(\text { letting } \theta=\sqrt{1+T_{c}^{2}} \zeta\right) \\
=\frac{1}{\left(1+T_{c}^{2}\right)^{n / 2}} \int_{-1}^{1} \frac{\theta_{n} d \theta_{n}}{\sqrt{1-\theta_{n}^{2}}\left(1+\theta_{n}\right)^{2}} \\
\quad \int_{\theta_{1}^{2}+\cdots+\theta_{n-1}^{2}=1-\theta_{n}^{2}} \theta_{i}^{2}\left(\theta_{j}^{2}-\frac{1}{n+2}\right) d \theta_{1} \cdots d \theta_{n-1} \\
=\frac{1}{\left(1+T_{c}^{2}\right)^{n / 2}} \int_{-1}^{1} \frac{\left(1-\theta_{n}^{2}\right)^{\frac{n-1}{2}} \theta_{n} d \theta_{n}}{\left(1+\theta_{n}\right)^{2}} \\
\quad\left\{\left(1-\theta_{n}^{2}\right) \int_{\mathbf{S}^{n-2}}^{\left(\operatorname{letting} \theta_{i}=\sqrt{1-\theta_{n}^{2}} \xi_{i}, 1 \leq i \leq n-1\right)} \frac{\left.\xi_{i}^{2} \xi_{j}^{2}-\frac{1}{n+2} \int_{\mathbf{S}^{n-2}} \xi_{i}^{2}\right\}}{=}\right. \\
=\frac{1}{\left(1+T_{c}^{2}\right)^{n / 2}} \int_{-1}^{1} \frac{\left(1-\theta_{n}^{2}\right)^{\frac{n-1}{2}} \theta_{n} d \theta_{n}}{\left(1+\theta_{n}\right)^{2}}\left\{\left(1-\theta_{n}^{2}\right) \frac{\sigma_{n-2}}{n^{2}-1}-\frac{1}{n+2} \frac{\sigma_{n-2}}{n-1}\right\} \\
=\frac{\sigma_{n-2}}{\left(1+T_{c}^{2}\right)^{n / 2}} \int_{-1}^{1} \frac{\left(1-\theta_{n}^{2}\right)^{\frac{n-1}{2}} \theta_{n} d \theta_{n}}{\left(1+\theta_{n}\right)^{2}}\left\{\frac{1}{\left(n^{2}-1\right)(n+2)}-\frac{\theta_{n}^{2}}{n^{2}-1}\right\}
\end{aligned}
$$


while

$$
\begin{aligned}
& \int_{\partial \Sigma} z_{i}^{2} \Psi_{3}^{(i)}=\int_{\partial \Sigma} \frac{\left(1+T_{c}^{2}\right)^{2} \zeta_{i}^{2}}{\left(1+\sqrt{1+T_{c}^{2}} \zeta_{n}\right)^{2}}\left(\zeta_{i}^{2}-\frac{1}{(n+2)\left(1+T_{c}^{2}\right)}\right) \zeta_{n} \\
&=\int_{\mathbf{S}^{n-1}} \frac{\theta_{i}^{2}\left(\theta_{i}^{2}-\frac{1}{n+2}\right) \theta_{n}}{\left(1+T_{c}^{2}\right)^{n / 2}\left(1+\theta_{n}\right)^{2}} d \theta \quad\left(\text { letting } \theta=\sqrt{1+T_{c}^{2}} \zeta\right) \\
&=\frac{1}{\left(1+T_{c}^{2}\right)^{n / 2}} \int_{-1}^{1} \frac{\theta_{n} d \theta_{n}}{\sqrt{1-\theta_{n}^{2}}\left(1+\theta_{n}\right)^{2}} \\
& \int_{\theta_{1}^{2}+\cdots+\theta_{n-1}^{2}=1-\theta_{n}^{2}} \theta_{i}^{2}\left(\theta_{i}^{2}-\frac{1}{n+2}\right) d \theta_{1} \cdots d \theta_{n-1} \\
&= \frac{1}{\left(1+T_{c}^{2}\right)^{n / 2}} \int_{-1}^{1} \frac{\left(1-\theta_{n}^{2}\right)^{\frac{n-1}{2}} \theta_{n} d \theta_{n}}{\left(1+\theta_{n}\right)^{2}} \\
&\left\{\left(1-\theta_{n}^{2}\right) \int_{\mathbf{S}^{n-2}}^{\xi_{i}^{4}-\frac{1}{n+2} \int_{\mathbf{S}^{n-2}}} \xi_{i}^{2}\right\} \\
&= \frac{1}{\left(1+T_{c}^{2}\right)^{n / 2}} \int_{-1}^{1} \frac{\left(1-\theta_{n}^{2}\right)^{\frac{n-1}{2}} \theta_{n} d \theta_{n}}{\left(1+\theta_{n}\right)^{2}}\left\{\left(1-\theta_{n}^{2}\right) \frac{3 \sigma_{n-2}}{n^{2}-1}-\frac{1}{n+2} \frac{\sigma_{n-2}}{n-1}\right\} \\
&= \frac{\sigma_{n-2}}{\left(1+T_{c}^{2}\right)^{n / 2}} \int_{-1}^{1} \frac{\left(1-\theta_{n}^{2}\right)^{\frac{n-1}{2}} \theta_{n} d \theta_{n}}{\left(1+\theta_{n}\right)^{2}}\left\{\frac{2 n+5}{\left(n^{2}-1\right)(n+2)}-\frac{3 \theta_{n}^{2}}{n^{2}-1}\right\} .
\end{aligned}
$$

Putting these together, we have

$$
\begin{aligned}
\int_{\partial \Sigma}\left(\sum_{i=1}^{n-1} \lambda_{i} z_{i}^{2}\right) \Psi_{3}^{(j)} \\
=\left(\sum_{i \neq j} \lambda_{i}\right) \frac{\sigma_{n-2}}{\left(1+T_{c}^{2}\right)^{n / 2}} \int_{-1}^{1} \frac{\left(1-\theta_{n}^{2}\right)^{\frac{n-1}{2}} \theta_{n} d \theta_{n}}{\left(1+\theta_{n}\right)^{2}} \\
\quad\left\{\frac{1}{\left(n^{2}-1\right)(n+2)}-\frac{\theta_{n}^{2}}{n^{2}-1}\right\} \\
+\lambda_{j} \frac{\sigma_{n-2}}{\left(1+T_{c}^{2}\right)^{n / 2}} \int_{-1}^{1} \frac{\left(1-\theta_{n}^{2}\right)^{\frac{n-1}{2}} \theta_{n} d \theta_{n}}{\left(1+\theta_{n}\right)^{2}}\left\{\frac{2 n+5}{\left(n^{2}-1\right)(n+2)}-\frac{3 \theta_{n}^{2}}{n^{2}-1}\right\} \\
=\frac{2 \lambda_{j} \sigma_{n-2}}{\left(n^{2}-1\right)\left(1+T_{c}^{2}\right)^{\frac{n}{2}}} \int_{-1}^{1} \frac{\left(1-\theta_{n}^{2}\right)^{\frac{n+1}{2}} \theta_{n} d \theta_{n}}{\left(1+\theta_{n}\right)^{2}} \\
=\frac{2^{n+1} \lambda_{j} \sigma_{n-2}}{\left(n^{2}-1\right)\left(1+T_{c}^{2}\right)^{\frac{n-2}{2}}} \int_{0}^{1} t^{\frac{n+1}{2}}(1-t)^{\frac{n-3}{2}}(1-2 t) d t
\end{aligned}
$$




$$
\begin{aligned}
& \left.\quad \text { (letting } 1-\theta_{n}=2 t\right) \\
& =\frac{2^{n+1} \lambda_{j} \sigma_{n-2}}{\left(n^{2}-1\right)\left(1+T_{c}^{2}\right)^{\frac{n}{2}}}\left\{B\left(\frac{n+3}{2}, \frac{n-1}{2}\right)-2 B\left(\frac{n+5}{2}, \frac{n-1}{2}\right)\right\} \\
& =\frac{2^{n+1} \lambda_{j} \sigma_{n-2}}{\left(n^{2}-1\right)\left(1+T_{c}^{2}\right)^{\frac{n}{2}}} \\
& \left.=-\frac{n+1}{4 n} B\left(\frac{n-1}{2}, \frac{n-1}{2}\right)-\frac{n+3}{4 n} B\left(\frac{n-1}{2}, \frac{n-1}{2}\right)\right\} \\
& =-\frac{2^{n} \lambda_{j} \sigma_{n-2}}{(n-1) n(n+1)\left(1+T_{c}^{2}\right)^{\frac{n}{2}}} B\left(\frac{n-1}{2}, \frac{n-1}{2}\right) \\
& 4 \lambda_{j} \sigma_{n-1}
\end{aligned}
$$

So

$$
\begin{aligned}
& \int_{\partial \Sigma}\left|f^{(3)}\right|^{2}= \frac{(n+1)(n-2)^{2}}{2^{n+2}(n+2)} \sum_{j=1}^{n-1}\left(\frac{4 \lambda_{j} \sigma_{n-1}}{(n-1) n(n+1)\left(1+T_{c}^{2}\right)^{\frac{n}{2}}}\right)^{2} \\
& \frac{(n+4)(n+2)^{2} n\left(1+T_{c}^{2}\right)^{\frac{n+5}{2}}}{2(n+1) \sigma_{n-1}} \\
&=\left(\sum_{j=1}^{n-1} \lambda_{j}^{2}\right) \frac{(n+4)(n+2)(n-2)^{2} \sigma_{n-1}}{2^{n-1}(n-1)^{2} n(n+1)^{2}\left(1+T_{c}^{2}\right)^{\frac{n-5}{2}}}
\end{aligned}
$$

We also write $Q_{3}$ in a similar form. First,

$$
\begin{aligned}
\int_{0}^{\infty} \frac{r^{n}}{\left(1+r^{2}\right)^{n-1}} d r & =\int_{0}^{\pi / 2} \sin ^{n} \theta \cos ^{n-4} \theta d \theta \\
& \left.=\frac{1}{2} \int_{0}^{1} t^{\frac{n-1}{2}}(1-t)^{\frac{n-5}{2}} d t \quad \text { (letting } t=\sin ^{2} \theta\right) \\
& =\frac{1}{2} B\left(\frac{n+1}{2}, \frac{n-3}{2}\right) \\
& =\frac{n-1}{2(n-3)} B\left(\frac{n-1}{2}, \frac{n-1}{2}\right) .
\end{aligned}
$$

Thus

$$
Q_{3}=-T_{c}\left(1+T_{c}^{2}\right)^{\frac{3-n}{2}}\left(\sum_{i=1}^{n-1} \lambda_{i}^{2}\right) \frac{\sigma_{n-2}(n-2)^{2}}{16(n-1)(n-3)} B\left(\frac{n-1}{2}, \frac{n-1}{2}\right)
$$


The existence of conformal metrics with constant scalar curvature $\quad 855$

$$
=-T_{c}\left(1+T_{c}^{2}\right)^{\frac{3-n}{2}}\left(\sum_{i=1}^{n-1} \lambda_{i}^{2}\right) \frac{\sigma_{n-1}(n-2)^{2}}{2^{n+2}(n-1)(n-3)} .
$$

A sufficient condition for (3.8) to hold is

$$
\frac{\int_{\partial \Sigma}\left|f^{(2)}\right|^{2}}{\mu_{2}}+\frac{\int_{\partial \Sigma}\left|f^{(3)}\right|^{2}}{\mu_{3}}>2 Q_{3} .
$$

Observe now

$$
\frac{\int_{\partial \Sigma}\left|f^{(2)}\right|^{2}}{\mu_{2}}=\left(\sum_{j=1}^{n-1} \lambda_{j}^{2}\right) \frac{(n-2)^{2}(n+2) \sigma_{n-1}}{2^{n+1} n(n-1)^{2}\left(1+T_{c}^{2}\right)^{\frac{n-5}{2}} \mu_{2}}
$$

and

$$
\frac{\int_{\partial \Sigma}\left|f^{(3)}\right|^{2}}{\mu_{3}}=\left(\sum_{j=1}^{n-1} \lambda_{j}^{2}\right) \frac{(n+4)(n+2)(n-2)^{2} \sigma_{n-1}}{2^{n-1}(n-1)^{2} n(n+1)^{2}\left(1+T_{c}^{2}\right)^{\frac{n-5}{2}} \mu_{3}} .
$$

Thus (4.6) is equivalent to

$$
\frac{n+2}{n(n-1) \mu_{2}}+\frac{4(n+2)(n+4)}{(n-1) n(n+1)^{2} \mu_{3}}>\frac{-T_{c}}{(n-3)\left(1+T_{c}^{2}\right)} .
$$

We can now verify (4.7) to conclude the proof of Theorem 1.1 with the following two estimates on $\mu_{2}$ and $\mu_{3}$ :

$$
\begin{aligned}
& \mu_{2}<\frac{1+T_{c}^{2}}{\left|T_{c}\right|} \\
& \mu_{3}<2 \frac{1+T_{c}^{2}}{\left|T_{c}\right|},
\end{aligned}
$$

which are just (3.14) for $k=2,3$.

Conclusion of Theorem 1.1. Using (4.8), (4.9), and the observation that

$$
1>\frac{3(n+1)^{2}}{(n-3)(n+2)(n+4)}, \quad \text { when } n \geq 5
$$

we have

$$
\frac{n+2}{n(n-1) \mu_{2}}+\frac{4(n+2)(n+4)}{(n-1) n(n+1)^{2} \mu_{3}}
$$




$$
\begin{aligned}
& >\frac{n+2}{n(n-1)} \frac{\left|T_{c}\right|}{1+T_{c}^{2}}+\frac{4(n+2)(n+4)}{(n-1) n(n+1)^{2}} \frac{3(n+1)^{2}}{2(n-3)(n+2)(n+4)} \frac{\left|T_{c}\right|}{1+T_{c}^{2}} \\
& \geq\left\{\frac{n+2}{n(n-1)}+\frac{6}{(n-1) n(n-3)}\right\} \frac{\left|T_{c}\right|}{1+T_{c}^{2}} \\
& \geq \frac{1}{n-3} \frac{\left|T_{c}\right|}{1+T_{c}^{2}} .
\end{aligned}
$$

Thus the condition in Proposition 2.1 has been verified to hold and Theorem 1.1 is proved.

Remark 4.1. We remark that in the verification of (3.8), $\mu_{2}$ alone will not be enough.

\section{Appendix A.}

Suppose $\lambda_{1}(M)>0$. Let us use $\left\langle u, v>\equiv \int_{M}\left(\nabla u \nabla v+c(n) R_{g} u v\right)+\right.$ $\frac{n-2}{2} \int_{\partial M} h_{g} u v$ to denote the inner product of $H^{1}(M)$, and $\|u\|=\sqrt{\langle u, u\rangle}$ to denote the norm. We consider the following functional defined on $H^{1}(M)$ :

$$
\begin{aligned}
I(u)= & \frac{1}{2} \int_{M}\left(|\nabla u|^{2}+c(n) R_{g} u^{2}\right)+\frac{n-2}{4} \int_{\partial M} h_{g} u^{2}-\frac{(n-2)^{2}}{2} \int_{M}\left(u^{+}\right)^{\frac{2 n}{n-2}} \\
& -\frac{(n-2) c}{2(n-1)} \int_{\partial M}\left(u^{+}\right)^{\frac{2(n-1)}{n-2}} .
\end{aligned}
$$

It is easy to see that $I \in C^{2}\left(H^{1}(M), \mathbf{R}\right)$ and that $u$ satisfies (1.1) if and only if $I^{\prime}(u)=0, u \in H^{1}(M) \backslash\{0\}$.

Let

$$
\bar{u}\left(z^{\prime}, z_{n}\right)=\left(\frac{1}{1+\left|z^{\prime}\right|^{2}+\left|z_{n}-\bar{t}\right|^{2}}\right)^{\frac{n-2}{2}},
$$

where $\bar{t}=-c /(n-2)$. It is well known that $\bar{u}$ satisfies

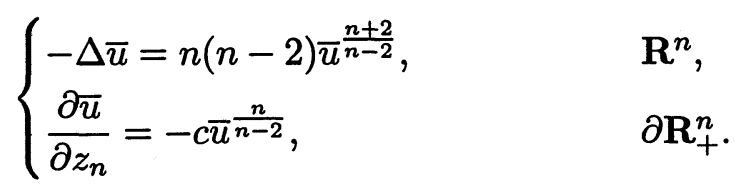

We define

$$
S_{c}=\frac{1}{2} \int_{\mathbf{R}_{+}^{n}}|\nabla \bar{u}|^{2}-\frac{(n-2)^{2}}{2} \int_{\mathbf{R}_{+}^{n}} \bar{u}^{\frac{2 n}{n-2}}-\frac{(n-2) c}{2(n-1)} \int_{\partial \mathbf{R}_{+}^{n}} \bar{u}^{\frac{2(n-1)}{n-2}},
$$


and

$$
S=\frac{1}{2} \int_{\mathbf{R}^{n}}|\nabla \bar{u}|^{2}-\frac{(n-2)^{2}}{2} \int_{\mathbf{R}^{n}} \bar{u}^{\frac{2 n}{n-2}} .
$$

It is not difficult to see that

$$
S=\frac{1}{n} \int_{\mathbf{R}^{n}}|\nabla \bar{u}|^{2}
$$

Lemma A.1. For all $c \in \mathbf{R}$,

$$
S-S_{c}=\frac{1}{2(n-1)} \int_{\mathbf{R}_{-}^{n}}|\nabla \bar{u}|^{2}+\frac{(n-2)^{2}}{2(n-1)} \int_{\mathbf{R}_{-}^{n}} \bar{u}^{\frac{2 n}{n-2}}>0 .
$$

Proof. Multiplying the equation of $\bar{u}$ by $\bar{u}$ and integrating by parts respectively on $\mathbf{R}_{+}^{n}$ and $\mathbf{R}_{-}^{n}$, we have

$$
\int_{\mathbf{R}_{+}^{n}}|\nabla \bar{u}|^{2}=n(n-2) \int_{\mathbf{R}_{+}^{n}} \bar{u}^{\frac{2 n}{n-2}}+c \int_{\partial \mathbf{R}_{+}^{n}} \bar{u}^{\frac{2(n-1)}{n-2}},
$$

and

$$
\int_{\mathbf{R}_{-}^{n}}|\nabla \bar{u}|^{2}+c \int_{\partial \mathbf{R}_{-}^{n}} \bar{u}^{\frac{2(n-1)}{n-2}}=n(n-2) \int_{\mathbf{R}_{-}^{n}} \bar{u}^{\frac{2 n}{n-2}} .
$$

It follows from (A.2) that

$$
S_{c}=\frac{1}{2(n-1)} \int_{\mathbf{R}_{+}^{n}}|\nabla \bar{u}|^{2}+\frac{(n-2)^{2}}{2(n-1)} \int_{\mathbf{R}_{+}^{n}} \bar{u}^{\frac{2 n}{n-2}} .
$$

It follows from (A.1) and (A.2) that

$$
\begin{aligned}
S-S_{c} & =\frac{1}{n} \int_{\mathbf{R}_{-}^{n}}|\nabla \bar{u}|^{2}+\frac{n-2}{2 n(n-1)} \int_{\mathbf{R}_{+}^{n}}|\nabla \bar{u}|^{2}-\frac{(n-2)^{2}}{2(n-1)} \int_{\mathbf{R}_{+}^{n}} \bar{u}^{\frac{2 n}{n-2}} \\
& =\frac{1}{2(n-1)} \int_{\mathbf{R}_{-}^{n}}|\nabla \bar{u}|^{2}+\frac{n-2}{2 n(n-1)} \int_{\mathbf{R}^{n}}|\nabla \bar{u}|^{2}-\frac{(n-2)^{2}}{2(n-1)} \int_{\mathbf{R}_{+}^{n}} \bar{u}^{\frac{2 n}{n-2}} \\
& =\frac{1}{2(n-1)} \int_{\mathbf{R}_{-}^{n}}|\nabla \bar{u}|^{2}+\frac{(n-2)^{2}}{2(n-1)} \int_{\mathbf{R}^{n}} \bar{u}^{\frac{2 n}{n-2}}-\frac{(n-2)^{2}}{2(n-1)} \int_{\mathbf{R}_{+}^{n}} \bar{u}^{\frac{2 n}{n-2}} \\
& =\frac{1}{2(n-1)} \int_{\mathbf{R}_{-}^{n}}|\nabla \bar{u}|^{2}+\frac{(n-2)^{2}}{2(n-1)} \int_{\mathbf{R}_{-}^{n}} \bar{u}^{\frac{2 n}{n-2}} .
\end{aligned}
$$

Lemma A.1 is established. 
Lemma A.2. Suppose $\lambda_{1}(M)>0$. Let $\left\{u_{i}\right\} \subset H^{1}(M)$ be a sequence of fuctions satisfying,

$$
I\left(u_{i}\right) \rightarrow b<S_{c}
$$

and

$$
\max _{v \in H^{1}(M) \backslash\{0\}} \frac{\left|I^{\prime}\left(u_{i}\right) v\right|}{\|v\|} \rightarrow 0 .
$$

Then after passing to a subsequence, either $\left\{u_{i}\right\}$ weakly converges in $H^{1}(M)$ to some solution of (1.1) or converges strongly to 0 in $H^{1}(M)$.

Proof. Take $v=u_{i}$ in (1.6), we have

$$
\left\|u_{i}\right\|^{2}-n(n-2) \int_{M}\left(u_{i}^{+}\right)^{\frac{2 n}{n-2}}-c \int_{\partial M}\left(u_{i}^{+}\right)^{\frac{2(n-1)}{n-2}}=\circ\left(\left\|u_{i}\right\|\right) .
$$

Multiplying (1.5) by $-2(n-1) /(n-2)$ and adding it to the above, we have

$$
\left\|u_{i}\right\|^{2}+(n-2)^{2} \int_{M}\left(u_{i}^{+}\right)^{\frac{2 n}{n-2}}=2(n-1) b+\circ(1)+\circ\left(\left\|u_{i}\right\|\right) .
$$

It follows immediately that

$$
\left\|u_{i}\right\| \leq C .
$$

Using the above and (1.6) with $v=u_{i}^{-}$, we have

$$
\left\|u_{i}^{-}\right\|^{2}=\circ(1) \text {. }
$$

It follows from (A.4) that after passing to some subsequence,

$$
u_{i} \rightarrow u \quad \text { weakly in } H^{1}(M),
$$

for some $u \in H^{1}(M)$. In view of (A.5), $u \geq 0$ a.e. on $M$. It follows from standard arguments that $I^{\prime}(u)=0$, namely,

$$
\begin{cases}-L_{g} u=n(n-2) u^{\frac{n+2}{n-2}}, & \text { on } M, \\ B_{g} u=c u^{\frac{n}{n-2}}, & \text { on } \partial M .\end{cases}
$$

If $u$ is not identically zero, then it follows from the Hopf lemma in its strong form (see e.g. lemma 3.4 of [GT]) that $u>0$ and therefore a solution of (1.1). So we assume in the following that $u \equiv 0$ and will use (1.5) to show by contradiction argument that

$$
\lim _{i \rightarrow \infty}\left\|u_{i}\right\|=0 .
$$


Suppose the contrary of (A.7), we have, along a subsequence, that

$$
\lim _{i \rightarrow \infty}\left\|u_{i}\right\|>0
$$

and therefore, in view of (A.3), (A.4) and (A.5),

$$
\lim _{i \rightarrow \infty}\left\{\int_{M} u_{i}^{\frac{2 n}{n-2}}+\int_{\partial M} u_{i}^{\frac{2(n-1)}{n-2}}\right\}>0 .
$$

As in $[\mathrm{L}]$, we define for $u_{i}$ the following concentration function:

$$
Q_{u_{i}}(r)=\max _{x \in M}\left\{\int_{B_{r}(x) \cap M}\left|u_{i}\right|^{\frac{2 n}{n-2}}+\int_{B_{r}(x) \cap \partial M}\left|u_{i}\right|^{\frac{2(n-1)}{n-2}}\right\} .
$$

Let $\epsilon>0$ be some small number to be determined later, and we define $r_{i}$ by

$$
Q_{u_{i}}\left(r_{i}\right)=\epsilon
$$

We first show that

$$
\lim _{i \rightarrow \infty} r_{i}=0 .
$$

Suppose the contrary of (A.10), we have $r_{i} \geq \bar{r}>0$ along a subsequence. Let $\bar{x}_{i} \in M$ be a point satisfying

$$
Q_{u_{i}}\left(r_{i}\right)=\int_{B_{r_{i}}\left(\bar{x}_{i}\right) \cap M}\left|u_{i}\right|^{\frac{2 n}{n-2}}+\int_{B_{r_{i}}\left(\bar{x}_{i}\right) \cap \partial M}\left|u_{i}\right|^{\frac{2(n-1)}{n-2}}=\epsilon
$$

and $\bar{x}_{i} \rightarrow \bar{x}$.

Let $\eta \in C^{\infty}(M)$ be some cutoff function with $\operatorname{diam}(\operatorname{supp} \eta)<\bar{r} / 2 \leq r_{i} / 2$ and take $v=\eta^{2}\left(u_{i}-u_{j}\right)$ in (1.6), we have, by using (A.5), (A.6) and the Sobolev embedding theorems, that

$$
\begin{aligned}
\circ(1)= & {\left[I^{\prime}\left(u_{i}\right)-I^{\prime}\left(u_{j}\right)\right]\left[\eta^{2}\left(u_{i}-u_{j}\right)\right] } \\
= & \int_{M} \nabla\left(u_{i}-u_{j}\right) \nabla\left(\eta^{2}\left(u_{i}-u_{j}\right)\right) \\
& -n(n-2) \int_{M}\left[\left|u_{i}\right|^{\frac{n+2}{n-2}}-\left|u_{j}\right|^{\frac{n+2}{n-2}}\right] \eta^{2}\left(u_{i}-u_{j}\right) \\
& -c \int_{\partial M}\left[\left|u_{i}\right|^{\frac{n}{n-2}}-\left|u_{j}\right|^{\frac{n}{n-2}}\right] \eta^{2}\left(u_{i}-u_{j}\right)+\circ(1) .
\end{aligned}
$$


Here and in the following $\circ(1)$ denotes some quantity which tends to zero as $i$ and $j$ tend to infinity. It follows, by using the mean value theorem, (A.9), (A.6) and $\operatorname{diam}(\operatorname{supp} \eta)<\bar{r} / 2 \leq r_{i} / 2$, that

$$
\begin{aligned}
\int_{M}\left|\nabla\left(\eta\left(u_{i}-u_{j}\right)\right)\right|^{2} \leq & C \int_{M}\left(\left|u_{i}\right|^{\frac{4}{n-2}}+\left|u_{j}\right|^{\frac{4}{n-2}}\right)\left[\eta\left(u_{i}-u_{j}\right)\right]^{2} \\
& +C \int_{\partial M}\left(\left|u_{i}\right|^{\frac{2}{n-2}}+\left|u_{j}\right|^{\frac{2}{n-2}}\right)\left[\eta\left(u_{i}-u_{j}\right)\right]^{2}+\circ(1) \\
\leq & C \epsilon^{\frac{2}{n}}\left\{\int_{M}\left[\eta\left(u_{i}-u_{j}\right)\right]^{\frac{2 n}{n-2}}\right\}^{\frac{n-2}{n}} \\
& +C \epsilon^{\frac{1}{n-1}}\left\{\int_{\partial M}\left[\eta\left(u_{i}-u_{j}\right)\right]^{\frac{2(n-1)}{n-2}}\right\}^{\frac{n-2}{n-1}}+\circ(1) \\
\leq & C \epsilon^{\frac{1}{n-1}} \int_{M}\left|\nabla\left(\eta\left(u_{i}-u_{j}\right)\right)\right|^{2}+\circ(1) .
\end{aligned}
$$

Consequently, if we fix $\epsilon>0$ at the beginning to satisfy $C \epsilon^{\frac{1}{n-1}}<1 / 2$, then

$$
\lim _{i, j \rightarrow \infty} \int_{M}\left|\nabla\left(\eta\left(u_{i}-u_{j}\right)\right)\right|^{2}=0 .
$$

It is easy to see from (A.6) with $u \equiv 0$ and the above that $u_{i} \rightarrow 0$ in $H^{1}(M)$ which contradicts to (A.8). This establishes (A.10).

Let $y^{1}, \cdots, y^{n}$ denote the geodesic normal coordinates given by some exponential map $\exp _{\bar{x}_{i}}$, and define $\widetilde{u}_{i}(z)=r_{i}^{(n-2) / 2} u_{i}\left(r_{i} z\right)$, for $z \in \tilde{M}_{i} \equiv$ $\left\{z \in \mathbf{R}^{n}: \exp _{\bar{x}_{i}}\left(r_{i} z\right) \in \bar{M},|z|<\delta_{0} / r_{i}\right\}$, where $\delta_{0}$ is half of the injectivity radius. Let $\tilde{g}$ denote the metric $\tilde{g}_{\alpha \beta} d z^{\alpha} d z^{\beta}$ with $\tilde{g}_{\alpha \beta}(z)=g_{\alpha \beta}\left(r_{i} z\right)$. It is easy to see, after passing to some subsequence, that there exists $R_{i} \rightarrow \infty$, $R_{i}<\delta_{0} /\left(10 r_{i}\right)$ such that

$$
\begin{aligned}
\lim _{i \rightarrow \infty}\left\{\int_{\left\{R_{i}<|z|<2 R_{i}\right\} \cap \tilde{M}_{i}}\left(\left|\nabla_{\tilde{g}} \tilde{u}_{i}\right|^{2}+\left|\tilde{u}_{i}\right|^{\frac{2 n}{n-2}}\right)\right. & \\
& \left.+\int_{\left\{R_{i}<|z|<2 R_{i}\right\} \cap \partial^{\prime} \tilde{M}_{i}}\left|\tilde{u}_{i}\right|^{\frac{2(n-1)}{n-2}}\right\}=0
\end{aligned}
$$

where $\partial^{\prime} \tilde{M}_{i}=\left\{z \in \mathbf{R}^{n}:|z|<\delta_{0} / r_{i}, \exp _{\bar{x}_{i}}\left(r_{i} z\right) \in \partial M\right\}$. Define some smooth cutoff function $\tilde{\eta}_{i}$ by

$$
\tilde{\eta}_{i}(z)= \begin{cases}1 & |z|<R_{i}, \\ 0 & |z|>2 R_{i},\end{cases}
$$


satisfying

$$
0 \leq \tilde{\eta}_{i}(z) \leq 1, \quad\left|\nabla \tilde{\eta}_{i}(z)\right| \leq C / R_{i}
$$

Set

$$
\tilde{u}_{i}^{(1)}=\tilde{\eta}_{i} \tilde{u}_{i}, \quad \tilde{u}_{i}^{(2)}=\tilde{u}_{i}-\tilde{u}_{i}^{(1)} .
$$

We also define on $M$

$$
u_{i}^{(1)}\left(\exp _{\bar{x}_{i}}(y)\right)=r_{i}^{-\frac{n-2}{2}} \tilde{u}_{i}^{(1)}\left(y / r_{i}\right)=\tilde{\eta}_{i}\left(y / r_{i}\right) u_{i}\left(\exp _{\bar{x}_{i}}(y)\right),
$$

and

$$
u_{i}^{(2)}=u_{i}-u_{i}^{(1)} .
$$

It is not difficult to see from (A.14) and (A.15) that

$$
\begin{aligned}
I\left(u_{i}\right) & =I\left(u_{i}^{(1)}\right)+I\left(u_{i}^{(2)}\right)+o(1), \\
I^{\prime}\left(u_{i}\right) & =I^{\prime}\left(u_{i}^{(1)}\right)+I^{\prime}\left(u_{i}^{(2)}\right)+o(1) \quad \text { in } H^{-1}(M),
\end{aligned}
$$

and

$$
\text { both } u_{i}^{(1)} \text { and } u_{i}^{(2)} \text { weakly converge to } 0 \text { in } H^{1}(M) \text {. }
$$

Writing

$$
b^{(1)}=\lim _{i \rightarrow \infty} I\left(u_{i}^{(1)}\right), \quad b^{(2)}=\lim _{i \rightarrow \infty} I\left(u_{i}^{(2)}\right) .
$$

It follows from (A.16) that

$$
b=b^{(1)}+b^{(2)} .
$$

We will first show that $b^{(2)} \geq 0$ and then show that $b^{(1)} \geq S_{c}$ to reach a contradiction.

Using (A.14), (A.15) and (1.6) with $v=u_{i}^{(1)}, u_{i}^{(2)}$ respectively, we have

$$
\begin{aligned}
& \circ(1)=I^{\prime}\left(u_{i}\right) u_{i}^{(1)}=I^{\prime}\left(u_{i}^{(1)}\right) u_{i}^{(1)}+\circ(1) \\
& \begin{aligned}
\int_{M}\left|\nabla u_{i}^{(1)}\right|^{2} & -n(n-2) \int_{M}\left|u_{i}^{(1)}\right|^{\frac{2 n}{n-2}}-c \int_{\partial M}\left|u_{i}^{(1)}\right|^{\frac{2(n-1)}{n-2}}+\circ(1), \\
\circ(1)= & I^{\prime}\left(u_{i}\right) u_{i}^{(2)}=I^{\prime}\left(u_{i}^{(2)}\right) u_{i}^{(2)}+\circ(1) \\
= & \int_{M}\left|\nabla u_{i}^{(2)}\right|^{2}-n(n-2) \int_{M}\left|u_{i}^{(2)}\right|^{\frac{2 n}{n-2}} \\
& -c \int_{\partial M}\left|u_{i}^{(2)}\right|^{\frac{2(n-1)}{n-2}}+\circ(1),
\end{aligned}
\end{aligned}
$$


Using (A.17) and (A.18), we have

$$
\begin{aligned}
b^{(2)}= & \frac{1}{2} \int_{M}\left|\nabla u_{i}^{(2)}\right|^{2}-\frac{(n-2)^{2}}{2} \int_{M}\left|u_{i}^{(2)}\right|^{\frac{2 n}{n-2}} \\
& -\frac{(n-2) c}{2(n-1)} \int_{\partial M}\left|u_{i}^{(2)}\right|^{\frac{2(n-1)}{n-2}}+\circ(1) \\
= & \left(\frac{1}{2}-\frac{n-2}{2(n-1)}\right) \int_{M}\left|\nabla u_{i}^{(2)}\right|^{2} \\
& +\left(\frac{n(n-2)^{2}}{2(n-1)}-\frac{(n-2)^{2}}{2}\right) \int_{M} \mid u_{i}^{\left.(2)\right|^{\frac{2 n}{n-2}}+\circ(1)} \\
\geq & \circ(1) .
\end{aligned}
$$

Therefore

$$
b^{(2)} \geq 0 \text {. }
$$

Let $\tilde{u}^{(1)}$ be the weak limit of $\tilde{u}_{i}^{(1)}$ in $H_{l o c}^{1}$. It folows from (A.5) that $\tilde{u}^{(1)} \geq 0$. For any test function $\tilde{\varphi} \in C_{c}^{\infty}\left(\mathbf{R}^{n}\right)$, set $\varphi(y)=r_{i}^{(2-n) / 2} \tilde{\varphi}\left(y / r_{i}\right)$. It is clear that

$$
\begin{aligned}
\circ(1)= & I^{\prime}\left(u_{i}\right) \varphi=I^{\prime}\left(u_{i}^{(1)}\right) \varphi+\circ(1)\|\varphi\| \\
= & \int_{\tilde{M}_{i}}\left(\nabla_{\tilde{g}} \tilde{u}_{i}^{(1)} \nabla_{\tilde{g}} \tilde{\varphi}+c(n) R_{\tilde{g}} \tilde{u}_{i}^{(1)} \tilde{\varphi}\right)+\frac{n-2}{2} \int_{\partial^{\prime} \tilde{M}_{i}} h_{\tilde{g}} \tilde{u}_{i}^{(1)} \tilde{\varphi} \\
& -n(n-2) \int_{\tilde{M}_{i}}\left(\tilde{u}_{i}^{(1)}\right)^{\frac{n+2}{n-2}} \tilde{\varphi}-c \int_{\partial^{\prime} \tilde{M}_{i}}\left(\tilde{u}_{i}^{(1)}\right)^{\frac{n}{n-2}} \tilde{\varphi}+\circ(1)\|\varphi\|
\end{aligned}
$$

Let $T=\lim _{i \rightarrow \infty} \operatorname{dist}\left(\bar{x}_{i}, \partial M\right) / r_{i}$. When $T=\infty$, we have from (A.19) that

$$
\int_{\mathbf{R}^{n}} \nabla \tilde{u}^{(1)} \nabla \tilde{\varphi}-n(n-2) \int_{\mathbf{R}^{n}}\left(\tilde{u}^{(1)}\right)^{\frac{n+2}{n-2}} \tilde{\varphi}=0
$$

namely,

$$
-\Delta \tilde{u}^{(1)}=n(n-2)\left(\tilde{u}^{(1)}\right)^{\frac{n+2}{n-2}}, \quad \mathbf{R}^{n}
$$

When $0 \leq T<\infty$, we have from (A.19) that

$$
\int_{\mathbf{R}_{-T}^{n}} \nabla \tilde{u}^{(1)} \nabla \tilde{\varphi}-n(n-2) \int_{\mathbf{R}_{-T}^{n}}\left(\tilde{u}^{(1)}\right)^{\frac{n+2}{n-2}} \tilde{\varphi}-c \int_{\partial \mathbf{R}_{-T}^{n}}\left(\tilde{u}^{(1)}\right)^{\frac{n}{n-2}} \tilde{\varphi}=0,
$$

namely,

$$
\left\{\begin{array}{lc}
-\Delta \tilde{u}^{(1)}=n(n-2)\left(\tilde{u}^{(1)}\right)^{\frac{n+2}{n-2}}, & z \in \mathbf{R}^{n}, z_{n}>-T \\
\frac{\partial \tilde{u}^{(1)}}{\partial z_{n}}=-c\left(\tilde{u}^{(1)}\right)^{\frac{n}{n-2}}, & z_{n}=-T
\end{array}\right.
$$


We see easily from (A.9) and (A.14) that

$$
Q_{\tilde{u}_{i}^{(1)}}(1) \equiv \sup _{z \in \tilde{M}_{i}}\left\{\int_{B_{1}(z) \cap \tilde{M}_{i}}\left|\tilde{u}_{i}^{(1)}\right|^{\frac{2 n}{n-2}}+\int_{B_{1}(z) \cap \partial^{\prime} \tilde{M}_{i}}\left|\tilde{u}_{i}^{(1)}\right|^{\frac{2(n-1)}{n-2}}\right\} \leq \epsilon+\circ(1) .
$$

Arguing as in (A.12) and (A.13), we know that $\left\{\tilde{u}_{i}^{(1)}\right\}$ strongly converges to $\tilde{u}^{(1)}$ in $H^{1}$ norm on any conpact sets. We aslo know from (A.11) and (A.14) that

$$
\int_{B_{1}(0) \cap \tilde{M}_{i}}\left|\tilde{u}_{i}^{(1)}\right|^{\frac{2 n}{n-2}}+\int_{B_{1}(0) \cap \partial^{\prime} \tilde{M}_{i}}\left|\tilde{u}_{i}^{(1)}\right|^{\frac{2(n-1)}{n-2}}=\epsilon+\circ(1) .
$$

It follows that $\tilde{u}^{(1)}$ is not identically zero. We can then apply the Liouville type theorems of Caffarelli-Gidas-Spruck [CGS] in $\mathbf{R}^{n}$ and Li-Zhu [LZ] in $\mathbf{R}_{+}^{n}$ to obtain the explicit forms of $\tilde{u}_{i}^{(1)}$ as follows.

When $T=\infty$, we have, for some $\tilde{\epsilon}>0, \tilde{z} \in \mathbf{R}^{n}$,

$$
\tilde{u}^{(1)}(z)=\left(\frac{\tilde{\epsilon}}{\tilde{\epsilon}^{2}+|z-\tilde{z}|^{2}}\right)^{\frac{n-2}{2}} \quad \text { on } \mathbf{R}^{n} .
$$

When $0 \leq T<\infty$, we have, for some $\tilde{\epsilon}>0, \tilde{z}=-c \tilde{\epsilon} /(n-2)$,

$$
\tilde{u}^{(1)}(z)=\left(\frac{\tilde{\epsilon}}{\tilde{\epsilon}^{2}+\left|z^{\prime}-\tilde{z}^{\prime}\right|^{2}+\left|z_{n}-\tilde{z}_{n}\right|^{2}}\right)^{\frac{n-2}{2}} \quad \text { on } \mathbf{R}_{-T}^{n} \text {. }
$$

It follows from (A.5) and (A.17) that $\left(u_{i}^{(1)}\right)^{-} \rightarrow 0$ in $H^{1}(M)$. It follows that

$$
\begin{aligned}
b^{(1)}= & \frac{1}{2} \int_{M}\left|\nabla u_{i}^{(1)}\right|^{2}-\frac{(n-2)^{2}}{2} \int_{M}\left(u_{i}^{(1)}\right)^{\frac{2 n}{n-2}} \\
& -\frac{(n-2) c}{2(n-1)} \int_{\partial M}\left(u_{i}^{(1)}\right)^{\frac{2(n-1)}{n-2}}+\circ(1),
\end{aligned}
$$

and

$$
\begin{aligned}
\circ(1) & =I^{\prime}\left(u_{i}\right) u_{i}^{(1)}=I^{\prime}\left(u_{i}^{(1)}\right) u_{i}^{(1)}+\circ(1) \\
& =\int_{M}\left|\nabla u_{i}^{(1)}\right|^{2}-n(n-2) \int_{M}\left(u_{i}^{(1)}\right)^{\frac{2 n}{n-2}}-c \int_{\partial M}\left(u_{i}^{(1)}\right)^{\frac{2(n-1)}{n-2}}+\circ(1) .
\end{aligned}
$$

Combining the above two estimates, we have

$$
b^{(1)}=\left(\frac{1}{2}-\frac{n-2}{2(n-1)}\right) \int_{M}\left|\nabla u_{i}^{(1)}\right|^{2}
$$


864

$$
+\left(\frac{n(n-2)^{2}}{2(n-1)}-\frac{(n-2)^{2}}{2}\right) \int_{M}\left(u_{i}^{(1)}\right)^{\frac{2 n}{n-2}}+\circ(1)
$$

Sending $i$ to $\infty$, we have

$$
\begin{aligned}
b^{(1)} \geq \begin{cases}\frac{1}{2(n-1)} \int_{\mathbf{R}^{n}}\left|\nabla \tilde{u}^{(1)}\right|^{2}+\frac{(n-2)^{2}}{2(n-1)} \int_{\mathbf{R}^{n}}\left(\tilde{u}^{(1)}\right)^{\frac{2 n}{n-2}} & \text { if } T=\infty, \\
\frac{1}{2(n-1)} \int_{\mathbf{R}_{-T}^{n}}\left|\nabla \tilde{u}^{(1)}\right|^{2}+\frac{(n-2)^{2}}{2(n-1)} \int_{\mathbf{R}_{-T}^{n}}\left(\tilde{u}^{(1)}\right)^{\frac{2 n}{n-2}} & \text { if } 0 \leq T<\infty\end{cases} \\
= \begin{cases}S & \text { if } T=\infty, \\
S_{c} & \text { if } 0 \leq T<\infty .\end{cases}
\end{aligned}
$$

In any case, we have shown that $b \geq b^{(1)} \geq S_{c}$ which contradicts to the hypothesis.

\section{Appendix B.}

In this Appendix, we provide the algebra which leads to the expansion (2.1). In the following, $\Delta a=a-a_{0}, \Delta b=b-b_{0}, \cdots$, etc.

$$
\begin{aligned}
\max _{0<t<\infty} I( & t u) \\
= & \frac{1}{2(n-1)}\left\{\left(a_{0}+\Delta a\right)(1+\Delta t)^{2}+(n-2)^{2}\left(b_{0}+\Delta b\right)(1+\Delta t)^{\frac{2 n}{n-2}}\right\} \\
= & \frac{1}{2(n-1)}\left\{\left(a_{0}+\Delta a\right)\left(1+2 \Delta t+(\Delta t)^{2}\right)\right. \\
& \left.+(n-2)^{2}\left(b_{0}+\Delta b\right)\left(1+\frac{2 n}{n-2} \Delta t+\left(\frac{n(n+2)}{(n-2)^{2}}+o(1)\right)(\Delta t)^{2}\right)\right\} \\
= & \frac{1}{2(n-1)}\left\{a_{0}+(n-2)^{2} b_{0}+\Delta a+\left(2 a_{0}+2 n(n-2) b_{0}\right) \Delta t\right. \\
& +(n-2)^{2} \Delta b+2 \Delta a \Delta t+2 n(n-2) \Delta b \Delta t \\
& \left.+\left[a_{0}+n(n+2) b_{0}\right](\Delta t)^{2}+o\left((\Delta t)^{2}\right)\right\} \\
= & S_{c}+\frac{1}{2(n-1)}\left\{\delta\left[A_{0}+\left(2 a_{0}+2 n(n-2) b_{0}\right) T_{0}+(n-2)^{2} B_{0}\right]+\right. \\
& +\epsilon \delta\left[A_{1}+(n-2)^{2} B_{1}+\left(2 a_{0}+2 n(n-2) b_{0}\right) T_{1}\right]+ \\
& +\delta^{2}\left[A_{2}+(n-2)^{2} B_{2}+\left(2 a_{0}+2 n(n-2) b_{0}\right) T_{2}+2 A_{0} T_{0}+\right. \\
& \left.+2 n(n-2) B_{0} T_{0}+\left(a_{0}+n(n+2) b_{0}\right) T_{0}^{2}\right]+ \\
& \left.+\epsilon^{2}\left[A_{3}+\left(2 a_{0}+2 n(n-2) b_{0}\right) T_{3}+(n-2)^{2} B_{3}\right]\right\}+o\left(\epsilon^{2}+\delta^{2}\right) .
\end{aligned}
$$


Observe that, using (1.4), we obtain $2 a_{0}+2 n(n-2) b_{0}=(n-2)\left[4 n b_{0}+\frac{2}{n-2} d_{0}\right]$. Using this and the expression for $T_{0}$, we simplify the coefficient of $\delta$ by

$$
\begin{aligned}
A_{0}+\left(2 a_{0}+2 n(n-2) b_{0}\right) T_{0}+ & (n-2)^{2} B_{0} \\
& =(n-1)\left(A_{0}-(n-2)^{2} B_{0}-\frac{n-2}{n-1} D_{0}\right) .
\end{aligned}
$$

Similarly we simplify the coefficients of $\epsilon \delta$ and $\epsilon^{2}$ by

$$
\begin{aligned}
A_{1}+\left(2 a_{0}+2 n(n-2) b_{0}\right) T_{1} & +(n-2)^{2} B_{1} \\
& =(n-1)\left(A_{1}-(n-2)^{2} B_{1}-\frac{n-2}{n-1} D_{1}\right), \\
A_{3}+\left(2 a_{0}+2 n(n-2) b_{0}\right) T_{3}+ & (n-2)^{2} B_{3} \\
& =(n-1)\left(A_{3}-(n-2)^{2} B_{3}-\frac{n-2}{n-1} D_{3}\right) .
\end{aligned}
$$

Finally the coefficient of $\delta^{2}$ is simplified as

$$
\begin{aligned}
& A_{2}+\left(2 a_{0}+2 n(n-2) b_{0}\right) T_{2}+(n-2)^{2} B_{2} \\
& \quad+2 T_{0}\left[A_{0}+n(n-2) B_{0}\right]+\left(a_{0}+n(n+2) b_{0}\right) T_{0}^{2} \\
& =(n-1)\left(A_{2}-(n-2)^{2} B_{2}-\frac{n-2}{n-1} D_{2}\right)+T_{0}\left(2 A_{0}-2 n(n-2) B_{0}-2 D_{0}\right) \\
& \quad+T_{0}^{2}\left(4 n b_{0}+\frac{2}{n-2} d_{0}\right)(n-3) \\
& =(n-1)\left\{A_{2}-(n-2)^{2} B_{2}-\frac{n-2}{n-1} D_{2}+\frac{\left[A_{0}-n(n-2) B_{0}-D_{0}\right]^{2}}{4 n b_{0}+\frac{2}{n-2} d_{0}}\right\} \\
& =(n-1)\left\{A_{2}-(n-2)^{2} B_{2}-\frac{n-2}{n-1} D_{2}\right. \\
& \left.\quad+\frac{n-2}{2 a_{0}+2 n(n-2) b_{0}}\left[A_{0}-n(n-2) B_{0}-D_{0}\right]^{2}\right\} .
\end{aligned}
$$

\section{Appendix C.}

In this Appendix, we sketch the elementary derivations for (2.6)-(2.8), (4.3)(4.4). First, (2.6) and (2.7) follow from direct integration by parts, and the last of (2.8) follows trivially from

$$
\int_{\mathbf{S}^{n-2}} \theta_{1}^{2}=\frac{1}{n-1} \int_{\mathbf{S}^{n-2}}\left(\theta_{1}^{2}+\cdots+\theta_{n-1}^{2}\right)=\frac{\sigma_{n-2}}{n-1} .
$$


Next, a change of variables shows that

$$
\begin{aligned}
\int_{\mathbf{S}^{n-2}} \theta_{1}^{2} \theta_{2}^{2} & =\int_{\mathbf{S}^{n-2}}\left(\frac{\theta_{1}+\theta_{2}}{\sqrt{2}}\right)^{2}\left(\frac{\theta_{1}-\theta_{2}}{\sqrt{2}}\right)^{2} \\
& =\frac{1}{4} \int_{\mathbf{S}^{n-2}}\left(\theta_{1}^{2}-\theta_{2}^{2}\right)^{2} \\
& =\frac{1}{2} \int_{\mathbf{S}^{n-2}} \theta_{1}^{4}-\frac{1}{2} \int_{\mathbf{S}^{n-2}} \theta_{1}^{2} \theta_{2}^{2}
\end{aligned}
$$

from which we obtain

$$
\int_{\mathbf{S}^{n-2}} \theta_{1}^{4}=3 \int_{\mathbf{S}^{n-2}} \theta_{1}^{2} \theta_{2}^{2}
$$

On the other hand,

$$
\begin{aligned}
\sigma_{n-2} & =\int_{\mathbf{S}^{n-2}}\left(\theta_{1}^{2}+\cdots+\theta_{n-1}^{2}\right)^{2} \\
& =(n-1) \int_{\mathbf{S}^{n-2}} \theta_{1}^{4}+(n-1)(n-2) \int_{\mathbf{S}^{n-2}} \theta_{1}^{2} \theta_{2}^{2},
\end{aligned}
$$

which, combining with (C.1), gives (2.8). Similarly, using

$$
\int_{\mathbf{S}^{n-1}} \theta_{1}^{6}=\int_{\mathbf{S}^{n-1}}\left(\frac{\theta_{1}+\theta_{2}}{\sqrt{2}}\right)^{6}
$$

we obtain the relation

$$
\int_{\mathbf{S}^{n-1}} \theta_{1}^{6}=5 \int_{\mathbf{S}^{n-1}} \theta_{1}^{4} \theta_{2}^{2}
$$

Together with

$$
\begin{aligned}
\int_{\mathbf{S}^{n-1}} \theta_{1}^{4} \theta_{2}^{2} & =\frac{1}{n-1} \int_{\mathbf{S}^{n-1}} \theta_{1}^{4}\left(\theta_{2}^{2}+\cdots+\theta_{n}^{2}\right) \\
& =\frac{1}{n-1} \int_{\mathbf{S}^{n-1}} \theta_{1}^{4}\left(1-\theta_{1}^{2}\right) \\
& =\frac{1}{n-1} \int_{\mathbf{S}^{n-1}} \theta_{1}^{4}-\frac{1}{n-1} \int_{\mathbf{S}^{n-1}} \theta_{1}^{6}
\end{aligned}
$$

we obtain (4.3). Finally,

$$
\int_{\mathbf{S}^{n-1}} \theta_{1}^{2} \theta_{2}^{2} \theta_{3}^{2}=\frac{1}{n-2} \int_{\mathbf{S}^{n-1}} \theta_{1}^{2} \theta_{2}^{2}\left(\theta_{3}^{2}+\cdots+\theta_{n}^{2}\right)
$$




$$
\begin{aligned}
& =\frac{1}{n-2} \int_{\mathbf{S}^{n-1}} \theta_{1}^{2} \theta_{2}^{2}\left(1-\theta_{1}^{2}-\theta_{2}^{2}\right) \\
& =\frac{1}{n-2} \int_{\mathbf{S}^{n-1}}\left(\theta_{1}^{2} \theta_{2}^{2}-2 \theta_{1}^{4} \theta_{2}^{2}\right) \\
& =\frac{\sigma_{n-1}}{n(n+2)(n+4)}
\end{aligned}
$$

using (2.8) and (4.3).

Acknowledgement. The first author would like to thank Professor Gang Tian for giving him the opportunity to visit the Math Department of MIT, and Professor Luen-Fai Tam for inviting him to visit the Math Department of the Chinese University of Hong Kong. Part of the work was completed during his visits to the above institutions.

\section{References.}

[AR] A. Ambrosetti and P.H. Rabinowitz, Dual variational methods in critical point theory and applications, J. Funct. Anal. 14 (1973), 349-381.

[A] T. Aubin, Équations différentielles non linéaires et probléme de Yamabe concernant la courbure scalaire, J. Math. Pures Appl. 55 (1976), 269-296.

[B] A. Bahri, Proof of the Yamabe conjecture without the positive mass conjecture for locally conformally flat manifolds, Nonlinear variational problems and partial differential equations (Isola d'Elba, 1990), 13-43, Pitman Res. Notes Math. Ser., 320, Longman Sci. Tech., Harlow, 1995.

[BB] A. Bahri and H. Brezis, Non-linear elliptic equations on Riemannian manifolds with the Sobolev critical exponent Topics in geometry, 1-100, Progr. Nonlinear Differential Equations Appl., 20, Birkhduser Boston, Boston, MA, 1996.

[BN] H. Brezis and L. Nirenberg, Positive solutions of nonlinear elliptic equations involving critical Sobolev exponents, Comm. Pure A ppl. Math. 36 (1983), 437-477.

[CGS] L. Caffarelli, B. Gidas and J. Spruck, Asymptotic symmetry and local behavior of semilinear elliptic equations with critical Sobolev growth, Comm. Pure Appl. Math. 42 (1989), 271-297.

[C] P. Cherrier, Problèmes de Neumann nonlinéaires sur les variétés Riemanniennes, J. Func. Anal. 57 (1984), 154-207. 
[E1] J.F. Escobar, Uniqueness theorems on conformal deformation of metric, Sobolev inequalities, and an eigenvalue estimate, Comm. Pure Appl. Math. 43 (1990), 857-883.

[E2] J. Escobar, The Yamabe problem on manifolds with boundary, J. Diff. Geom. 35 (1992), 21-84.

[E3] J. Escobar, Conformal deformation of a Riemannian metric to a scalar flat metric with constant mean curvature, Ann. of Math. 136 (1992), 1-50.

[E4] J. Escobar, Conformal deformation of a Riemannian metric to a constant scalar curvature metric with constant mean curvature on the boundary, Indiana Univ. Math. J. 45 (1996), 917-943.

[E5] J. Escobar, Conformal metrics with prescribed mean curvature on the boundary, Calc. Var. and PDEs 4 (1996), 559-592.

[GT] D. Gilbarg and N. S. Trudinger, Elliptic Partial Differential Equations of Second Order, 2nd ed., Grundlehren der Mathematischen Wissenschaften [Fundamental Principles of Mathematical Sciences] No. 224, SpringerVerlag, Berlin-New York, 1983.

[HL1] Z.C. Han and Y.Y. Li, The Yamabe problem on manifolds with boundaries: Existence and compactness results, Duke Mathematical Journal, 99 (1999), 489-542.

[HL3] Z.C. Han and Y.Y. Li, Further results on the Yamabe problem with boundary, in preparation.

[LP] J. Lee and T. Parker, The Yamabe problem, Bull. Amer. Math. Soc. 17 (1987), 37-91.

[LZ] Y.Y. Li and M. Zhu, Uniqueness theorems through the method of moving spheres, Duke Math. J. 80 (1995), 383-417.

[L] P. L. Lions, The concentration-compactness principle in the calculus of variations. The limit case, Part 2, Revista Matemática Iberoamericana 1 (1985), 45-121.

[S1] R. Schoen, Conformal deformation of a Riemannian metric to constant scalar curvature, J. Diff. Geom. 20 (1984), 479-495.

[S2] R. Schoen, Variational theory for the total scalar curvature functional for Riemannian metrics and related topics, in Topics in Calculus of Variations, Lecture notes in mathematics, No. 1365, edited by M. Giaquinta, SpringerVerlag, 1989, 120-154 
The existence of conformal metrics with constant scalar curvature $\quad 869$

[S3] R. Schoen, On the number of constant scalar curvature metrics in a conformal class, Differential Geometry: A symposium in honor of Manfredo Do Carmo (H.B. Lawson and K. Tenenblat, eds), Wiley, 1991, 311-320.

[SY1] R. Schoen and S.T. Yau, On the proof of the positive mass conjecture in General Relativity, Comm. Math. Phys. 65 (1979), 45-76.

[SY2] R. Schoen and S.T. Yau, Conformally flat manifolds, Kleinian groups, and scalar curvature, Invent. Math. 92 (1988), 47-71.

[T] N. Trudinger, Remarks concerning the conformal deformation of Riemannian structures on compact manifolds, Ann. Scuola Norm. Sup. Cl. Sci. (3) 22 (1968), 265-274.

[Y] H. Yamabe, On a deformation of Riemannian structures on compact manifolds, Osaka Math. J. 12 (1960), 21-37.

ReCeived June 18, 1998.

RUTGERS UNIVERSITY

NEW BRunswick, NJ 08903

USA 